\title{
Analysis of Infusion Pump Data from CHEO
}

\author{
by
}

\author{
Queeny Shaath, B. Eng.
}

\author{
A thesis submitted to the \\ Faculty of Graduate and Postdoctoral Affairs \\ in partial fulfillment of the requirements for the degree of
}

Master of Applied Science in Biomedical Engineering

\author{
Ottawa-Carleton Institute for Biomedical Engineering \\ Department of Systems and Computer Engineering \\ Carleton University \\ Ottawa, Ontario \\ April, 2014 \\ (C)Copyright \\ Queeny Shaath, 2014
}


The undersigned hereby recommends to the

Faculty of Graduate and Postdoctoral Affairs acceptance of the thesis

\title{
Analysis of Infusion Pump Data from CHEO
}

\author{
submitted by Queeny Shaath, B. Eng. \\ in partial fulfillment of the requirements for the degree of \\ Master of Applied Science in Biomedical Engineering
}

Professor Adrian D. C. Chan, Thesis Co-supervisor

Professor Doron Nussbaum, Thesis Co-supervisor

Kim Greenwood, Thesis Co-supervisor

Professors Hilmi Dajani and James Green, Readers

Professor Roshdy Hafez, Chair,

Department of Systems and Computer Engineering

Ottawa-Carleton Institute for Biomedical Engineering

Department of Systems and Computer Engineering

Carleton University

April, 2014 


\begin{abstract}
The thesis objective is to analyze smart infusion pump data from the Children's Hospital of Eastern Ontario to learn about pump usage. Trends in compliance and hard limit events were shown to be potentially associated with drug library updates, and can be used to assess library changes. In a case study for the drug gentamicin, selection errors were the cause of more than $50 \%$ of hard limit events. A human computer interaction issue was identified as the problem, where gentamicin has two library entries that are named in a manner that may be confusing. Decision trees were used to determine factors that are associated with hard limits. Results showed that higher hard limit event rates were associated with the NICU and Emergency profiles, and with patients who weighed over $48 \mathrm{~kg}$; these factors can be used to target training and research. This thesis contributes to research in medication errors and patient safety.
\end{abstract}




\section{Acknowledgments}

I would like to offer my sincerest gratitude to my three co-supervisors: Dr.Adrian Chan for his constant support, encouragement and guidance throughout this research, Kim Greenwood for his invaluable mentorship and support, and Dr.Doron Nussbaum for his insightful feedback and contributions. Thank you for always being available to answer all my questions.

This work would not have been possible without the help, feedback, and insight from several CHEO members: Dr.Regis Vaillancourt, Mary MacNeil, Carmen Ma, Dr.Antoinette Corvo, Mark Asbil, Rachel Zhang and Marie-Ange Janvier. Thank you for your trust and support in my work.

A complete understanding of the pump and the PharmGuard database would not have been possible without the help and support from the Smiths Medical team and especially from Jessica Hagg. Thank you for taking the time to promptly and thoroughly answer all of my e-mails.

My time as a Master's student would not have been the same without my friends and colleagues on the 6th floor of Canal building. I appreciate all your encouragement and help. Alicia Gal, Daphne Ong, Justin Wagenaar, and Sarah Girgrah: thank you for taking the time to help me practice for my defence presentation- it would not have been as successful without you.

To my parents, thank you for encouraging me to do my best, for all your patience and trust in me. To my brother and sister, thank you for your love and inspiration. To my best friends Anjali and James, thank you for your positivity, and constant presence. 


\section{Table of Contents}

Abstract

Acknowledgments $\quad$ iv

Table of Contents $\quad$ v

List of Tables $\quad$ viii

List of Figures $\quad$ ix

Nomenclature $\quad$ xi

1 Introduction $\quad 1$

1.1 Background . . . . . . . . . . . . . . . . . . . . 1

1.2 Motivation ....................... . . 4

1.3 Thesis objectives $\ldots \ldots \ldots \ldots \ldots \ldots \ldots \ldots \ldots$

1.4 Contributions . . . . . . . . . . . . . . . . . 6

1.5 Thesis organization $\ldots \ldots \ldots \ldots \ldots \ldots \ldots$

2 Background $\quad 10$

2.1 Introduction to medical errors . . . . . . . . . . . . . . 10

2.1 .1 Infusion pumps . . . . . . . . . . . . . . . . . . 13

2.1.2 Medication errors and infusion pumps . . . . . . . . . . 16

2.1.3 Medication errors and smart pumps . . . . . . . . . . . . . 17

2.1.4 Drug library research . . . . . . . . . . . . . . . . . 21

2.1.5 Proposed solutions for reducing infusion pump errors in research 22

2.1.6 Regulatory activity . . . . . . . . . . . . . . . . 22

2.2 Introduction to data mining . . . . . . . . . . . . . . . . . 24

2.2 .1 Mushroom data . . . . . . . . . . . . . . . . . . 26

2.2 .2 Decision tree methodology . . . . . . . . . . . . . . . . . 29

2.2 .3 Split criterion . . . . . . . . . . . . . . . . . . 33

2.2 .4 Stop criterion . . . . . . . . . . . . . . . 36

2.2.5 Method for applying the decision tree technique to mushroom data . . . . . . . . . . . . . . . 37 
2.2.6 Decision tree results and discussion using mushroom data . . . 39

2.2.7 Conclusions . . . . . . . . . . . . . . . . . 42

3 Analysis of Smart Pump Usage at CHEO 43

3.1 Introduction . . . . . . . . . . . . . . . . . 43

3.2 The Children's Hospital of Eastern Ontario . . . . . . . . . . . . . . . 44

3.3 Smart pumps at CHEO . . . . . . . . . . . . . . . . . 46

3.3.1 Drug library updates . . . . . . . . . . . . . . . 47

3.3.2 Prescriptions . . . . . . . . . . . . . . . . . . 47

3.3.3 Profiles and profile categories . . . . . . . . . . . . . 48

3.4 Smiths Medical's Medfusion 4000 syringe infusion pump . . . . . . . 49

3.4.1 Compliance . . . . . . . . . . . . . . . 50

3.4.2 Drug library updates . . . . . . . . . . . . . . . . . . . . . 52

3.4.3 Programming an infusion . . . . . . . . . . . . 53

3.4.4 The Event History report . . . . . . . . . . . . . . . . . . 60

3.5 Stakeholders . . . . . . . . . . . . . . . . . 62

3.6 Examining compliance and hard limit trends at $\mathrm{CHEO}$. . . . . . . 64

3.6.1 Methodology . . . . . . . . . . . . . . 64

3.6.2 Results and discussion . . . . . . . . . . . . . . . . . 66

3.6.3 Conclusions ....................... 79

4 Gentamicin Case Study $\quad 81$

4.1 Introduction . . . . . . . . . . . . . . . . . . . 81

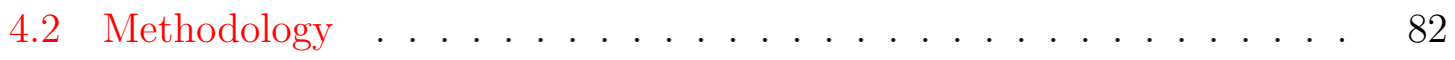

4.2.1 Hard limit event follow-up actions . . . . . . . . . . . . . 83

4.2.2 Hard limit event error . . . . . . . . . . . . . . . 84

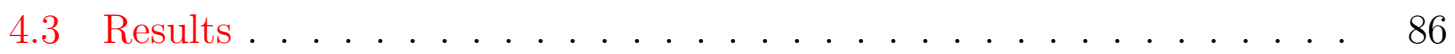

4.4 Discussion . . . . . . . . . . . . . . . . . . . . . . 87

4.5 Conclusions . . . . . . . . . . . . . . . . . . . . 90

5 Analyzing Infusion Data Using Decision Trees 91

5.1 Introduction to the infusion data research question . . . . . . . . . . 92

5.2 Objective . . . . . . . . . . . . . . . . . . . 92

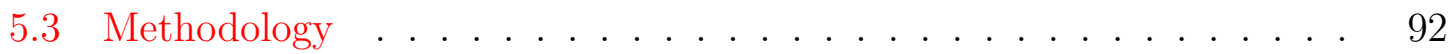

5.3.1 Infusion data description and criteria . . . . . . . . . . . . . . 93

5.3.2 Infusion data decision tree criteria . . . . . . . . . . . . 96

5.4 Decision tree results for infusion data . . . . . . . . . . . . . . 97

5.5 Discussion . . . . . . . . . . . . . . . . . . 102

5.5.1 Analysis of errors in the emergency department . . . . . . . . 104

5.5.2 Analysis of errors in the neonatal intensive care unit . . . . . 105

5.5.3 Contribution to trigger-based research . . . . . . . . . . 106

5.6 Conclusions . . . . . . . . . . . . . . . . 106 
6 Summary 108

6.1 Conclusions . . . . . . . . . . . . . . . . . . . . . . . . 108

6.2 Future work and recommendations . . . . . . . . . . . 110

$\begin{array}{ll}\text { List of References } & 116\end{array}$

Appendix A Event History Report Events 124 


\section{List of Tables}

2.1 List of 21 variables and possible values for mushroom data set. . . . .

2.2 Impurity measures calculated using gini and deviance split criteria for the branches in Figure 2.5 . . . . . . . . . . . . . . . . 36

3.1 Profiles in the CHEO drug library with description . . . . . . . . . . 49

3.2 Hard limit event and infusion statistics by month: total infusions, $I_{T}$; PharmGuard infusions, $I_{P G}$; non-PharmGuard infusions, $I_{n P G}$; total hard limit events, $H L_{T}$; total hard limit events followed by a non-

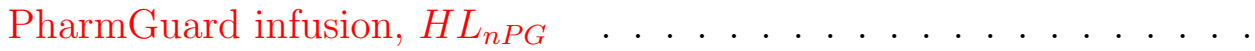

3.4 Hard limit event and infusion statistics by profile: total infusions, $I_{T}$; PharmGuard infusions, $I_{P G}$; non-PharmGuard infusions, $I_{n P G}$; total hard limit events, $H L_{T}$; total hard limit events followed by a nonPharmGuard infusion, $H L_{n P G}$. . . . . . . . . . . . . . . . .

3.5 Infusion statistics by month for the Hem/Onc profile from August 2011 to November 2012: total infusions, $I_{T}$; PharmGuard infusions, $I_{P G}$; non-PharmGuard infusions, $I_{n P G} \ldots \ldots$. . . . . . . . .

3.6 Infusion statistics by month for the Anesthesia CVS profile from August 2011 to August 2012: total infusions, $I_{T}$; PharmGuard infusions, $I_{P G}$; non-PharmGuard infusions, $I_{n P G} \ldots \ldots$. . . . . . . 70

4.1 Hard limit event follow up actions. . . . . . . . . . . . . . . 86

4.2 User errors. . . . . . . . . . . . . . . . . . . . . . . . 87

5.1 Infusion parameters. . . . . . . . . . . . . . . . . . 94

5.2 Inclusion and exclusion criteria for infusion data. . . . . . . . . . . 96

5.3 Infusion data variables used to build infusion decision trees for NoHL vs. HL infusions. . . . . . . . . . . . . . . . . . . . . . . 96

5.4 Infusion gini tree class count, percent of total and percent of node calculated at each node . . . . . . . . . . . . . . . . . 99

5.5 Infusion deviance tree class count, percent of total and percent of node calculated at each node . . . . . . . . . . . . . . . . . . . 100 


\section{List of Figures}

1.1 Medfusion 4000 syring infusion pump . . . . . . . . . . . . 3

2.1 Basic infusion pump and components: 1) fluid reservoir, 2) catheter system, and 3) electronic interface. . . . . . . . . . . . . . 14

2.2 Classification rules for classifying poisonous and edible mushrooms presented in chart form. . . . . . . . . . . . . . . . . .

2.3 All the possible splits for a categorical color variable with level values of blue and red . . . . . . . . . . . . . . . . 32

2.4 Gini and deviance curves for different fractions of $X_{1} \ldots \ldots \ldots$

2.5 Decision tree splits with gini criterion (a) and deviance criterion (b) .

2.6 Mushroom classification rules converted into a binary univariate decision tree . . . . . . . . . . . . . . . . . . 38

2.7 Mushroom tree built with the gini criterion . . . . . . . . . 40

2.8 Mushroom tree built with the deviance criterion . . . . . . . . . 41

3.1 Medfusion 4000 Pump . . . . . . . . . . . . . . . . . . . . . . 44

3.2 Pump programming steps 2-6: Images taken of the Medfusion 4000 for programming a "gentamicin $5 \mathrm{mg} / \mathrm{mL}$ once daily" infusion in the General Peds/Surg profile . . . . . . . . . . . . . . . . . .

3.3 Pump programming steps 7-9: Images taken of the Medfusion 4000 for programming a "gentamicin $5 \mathrm{mg} / \mathrm{mL}$ once daily" infusion in the General Peds/Surg profile . . . . . . . . . . . . . . . . . .

3.4 Pump programming steps 4-7: Images taken of the Medfusion 4000 for programming a Volume-over-Time infusion . . . . . . . . . . . .

3.5 Example of hard limit event when entering infusion time. A hard limit event is the event where a clinician enters a value outside the Hard limit events in the drug library. . . . . . . . . . . . . . .

3.6 Example of Soft limit event override event when entering dose. A soft limit event is the event where a clinician enters a value that is outside of the soft limit but inside of the hard limits in the drug library. . . .

3.7 The percent of hard limit events over all infusions, $\left(\frac{H L_{T}}{I_{T}}\right)$, and the percent of hard limit events followed by a non-PharmGuard infusion over all hard limit events, $\left(\frac{H L_{n P G}}{H L_{T}}\right)$, for every month starting from August 2011 to August 2012 . . . . . . . . . . . . . . . . . . . . 
3.8 The percent of Hard limit events followed by a non-PharmGuard infusion, $\left(\frac{H L_{n P G}}{I_{T}}\right)$, and the percent of non-PharmGuard infusions over all infusions, $\left(\frac{I_{n P G}}{I_{T}}\right)$, from August 2011 to August $2012 \ldots \ldots \ldots \ldots . .74$

3.9 The percent of non-PG infusions over all infusion, $\left(\frac{I_{n P G}}{I_{T}}\right)$, and the percent of non-PG infusions that followed a Hard limit event, $\left(\frac{H L_{n P G}}{I_{n P G}}\right)$,for nine CHEO drug library profiles from August 2011 to August 2012. .

3.10 The percent of non-PharmGuard infusions over all infusions, $\left(\frac{I_{n P G}}{I_{T}}\right)$, by month for the Hem/Onc profile from August 2011 to November 201277

3.11 The percent of non-PharmGuard infusions over all infusions, $\left(\frac{I_{n P G}}{I_{T}}\right)$, by month for the Anesthesia CVS profile from August 2011 to August

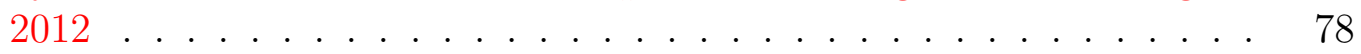

5.1 Infusion gini tree: Infusion tree built with the gini criterion . . . . . 97

5.2 Infusion deviance tree: Infusion tree built with the deviance criterion 98 


\section{Nomenclature}

\begin{tabular}{ll}
\hline Acronyms & Definition \\
\hline \hline General Peds/Surg & General pediatrics and surgery \\
Hem/onc & Hematology and oncology \\
Ane & Anesthesia \\
CVS & Cardiovascular surgery \\
Emerg & Emergency \\
MDU & Medical day unit \\
NICU & Neonatal intensive care unit \\
PICU & Pediatric intensive care unit \\
HL & Hard limit \\
NoHL & No hard limit \\
PG & PharmGuard \\
non-PG & Non-PharmGuard \\
\hline
\end{tabular}




\section{Chapter 1}

\section{Introduction}

\section{$1.1 \quad$ Background}

Errors that occur in the health care system place an unwanted risk on the patients involved. An error may occur that is never noticed because it has no effect on the patient; however, errors can also be quite serious, adding extra complications to a patient's condition, and may even lead to death. A recent study reviewed 523 death reports between 2007 and 2012 in four Canadian provinces that were suspected to be caused by a medication incident [1]. Of the 523 reviewed incidents, 115 were determined to have contributed to or resulted in the patient's death. Medical errors can be caused by device errors (e.g., defective equipment), system errors (e.g., discrepancies

in standards and hospital policies), medication errors (e.g., prescription errors), and user errors (e.g., problems with operating medical devices). Medical errors also have the effect of reducing satisfaction for both patient and care provider, and reducing the public's trust in the health care system.

Medication errors have the potential of occurring due to user errors when operating an infusion pump. (Figure 1.1 is a photo of a syringe volume pump). An infusion pump is a medical device designed to automate delivery of specific amounts of liquid 
drugs or other liquids into a patient over a period of time. To setup an infusion, a container holding the liquid (e.g., syringe or bag) must be appropriately installed on the infusion pump, and a catheter system must be properly setup to transfer the liquid from the container to the patient. Then, the medical user (nurse or doctor) must program the pump by entering a combination of dose, time and/or rate in order to administer the prescribed medication. A medication error may occur when the user makes a mistake when entering any one of the prescription variables into the pump. The following is an example of an actual pump programming error [2]:

A patient admitted in the emergency department was diagnosed with dyspnea, or difficulty breathing. The patient was a 19-year old woman who recently had a cesarean section birth, leading the doctor to suspect a pulmonary embolism (e.g., obstruction in an artery of the lungs). The patient was prescribed a 5000 unit bolus dose and 1000 units/hour (equal to $20 \mathrm{~mL} /$ hour) continuous infusion dose of IV heparin, a drug targeting blood clotting factors. The nurse administered the bolus dose, then programmed the pump for $1000 \mathrm{~mL} /$ hour (instead of units/hour) , fifty times more than the prescribed dose. The error was detected in less than hour, and had caused the patient's aPTT value, a measure of a part of the blood clotting system, to rise to 240 seconds (IV heparin treatment aims for a value between 45 to 70 seconds). Fortunately, the patient did not experience adverse bleeding.

Smart infusion pumps are the latest generation infusion pumps. These devices guide users in programming the pump, based on drug libraries, to help reduce drug programming errors. A drug library specifies infusion parameter ranges (e.g., dose, rate, time) based on hospital drug standards. Typically, two sets of limits are programmed: soft limits and hard limits. Soft limits define the range for normal use but may be exceeded if the clinical user confirms the entered infusion value and overrides 


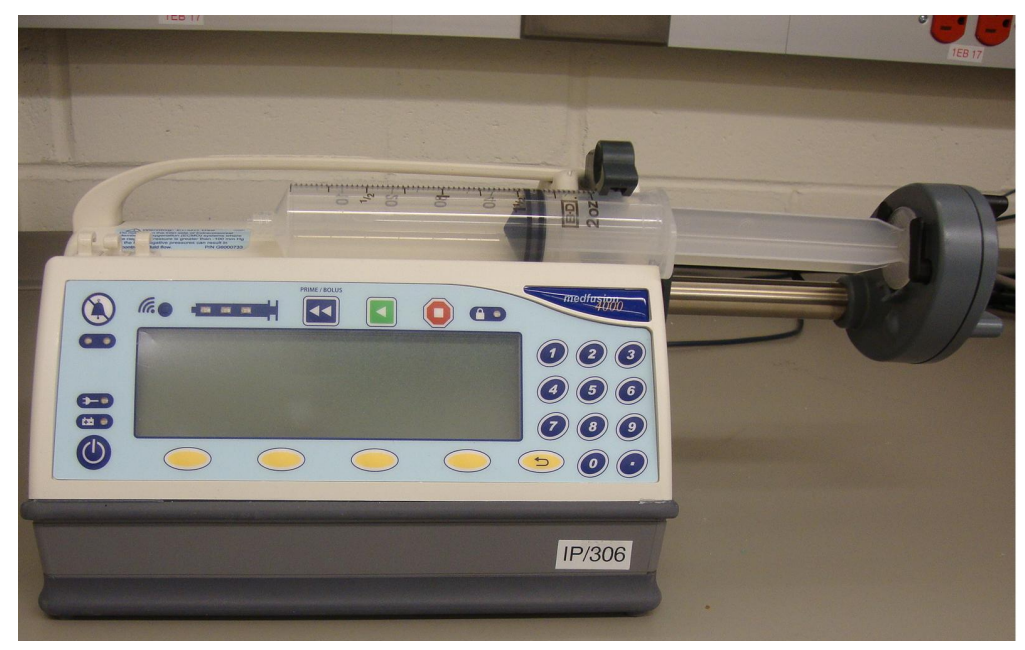

Figure 1.1: Medfusion 4000 syring infusion pump

the soft limit alert. Hard limits are limits that cannot be exceeded. Entering a value outside of the hard limit range is not accepted by the pump and the infusion will not be delivered until a value within the limits is re-entered. Whenever a value is entered that exceeds a hard or soft limit, a limit event saves the infusion parameters in a history log. Setting up an infusion outside of the drug library can be done by using the pump as a traditional pump, generically programming volume, rate and/or time; however, this bypasses the pump safety features that detect programming errors. Compliance is the use of the drug library when programming an infusion on a smart pump. Compliance and limit events are basic indicators of how the pumps are being used in a hospital. A medical error occurring because of any aspect of the infusion pump system (e.g., design, training, drug libraries) has the potential to affect a large number of patients in a hospital. 


\subsection{Motivation}

Data collected from smart infusion pumps give researchers the opportunity to study a medical device that a large number of people will be exposed to. Research and improvement of infusion pump systems will improve the services offered by the hospital by having a direct positive impact on the health and safety of all patients admitted into the hospital; this in turn will reduce health care costs resulting from negative outcomes of medication errors and allow reallocation of resources to improve other parts of the health care system and services.

The advancement of recent technologies in data collection, transfer and storage has allowed users from various industries, including the health care industry, to gather and save large amounts of data from various systems and processes [3]. The existence of this large amount of data, widely known as big data, has created a demand in the research and development of advanced data analytic methods to process the data and generate meaningful results that are easily interpreted. These results can be used to aid in the advancement of the system that generated the data in the first place [4]. Extracting useful information from big data manually is becoming impractical, if not impossible, which is instigating research in new automatic and semi-automatic methods. Smart infusion pumps are a relatively new technology in the health care industry that provide an opportunity for exploring data analytic methods as they generate a large amount of data that are potentially useful. Current research of the analysis of smart pump data has been limited, and the aim of this research is to contribute to this work. 


\subsection{Thesis objectives}

The main objective of this thesis is to analyze smart pump data from the Children's Hospital of Eastern Ontario (CHEO). Research outcomes could lead to new methods for data analytics for smart pump data to identify usability issues and usage issues

(e.g., compliance), to provide evidence on the value of the infusion pumps in detecting user errors, and to provide a means of performance evaluation (e.g., allows a feedback mechanism to evaluate the effectiveness of personnel training, can identify compliance issues by profile to focus training on problem profiles). In the long term, this could lead to more efficient use of smart pumps, improve patient safety by reducing drug errors, reduce costs and resources (from increased efficiency and lower drug error rate), and increase satisfaction for both patients and health care providers.

The thesis has been split into three sub-objectives. The first sub-objective of the thesis is to show how a basic analysis of trends in compliance and Hard limit events can be studied and linked to training and library updates. The second sub-objective is to show through a case study how information from the smart infusion pump can be used for designing better infusion pumps, improving drug libraries and improving training opportunities. The third sub-objective is to show how decision trees can be employed to discover factors associated with different infusion events. Identifying discerning factors can help target further research, library development, and training. In this research, decision trees are constructed to separate infusion types (infusions hitting hard limits and those that do not) and then analysis of the tree is used to identify factors favouring infusions associated with Hard limit events. Results can be used to improve the efficiency of smart infusion usage. 


\subsection{Contributions}

The contributions of this thesis are summarized below:

\section{Demonstrated how smart infusion pump data can be used to determine factors that influence compliance and to quantify the efficacy of compliance training}

Non-compliance rates were observed and compared for the different hospital departments (each hospital department is associated with one drug library profile). Non-compliance trends were also observed for one department, the hematology/oncology department, over a 16-month period. Comparing non-compliance rates of different departments showed that the hematology/oncology department and the cardiovascular surgery anesthesia department had the highest non-compliance rates during the study period. Though high non-compliance is a negative indicator, anesthesia is a unique practice where non-compliance may be more effective and does not put a patient at risk. When observing the non-compliance trends for the hematology/oncology department, non-compliance increased over a period of nearly a year, which is probably what instigated a training and information session in July 2012. Results of the trend in the following 4 months showed that noncompliance decreased after the training by nearly $20 \%$, indicating a positive outcome. This type of analysis provides the opportunity of evidence-based practice that can identify the need for intervention and that allows for an evaluation of the performance of interventions.

2. Demonstrated that basic infusion parameters such as compliance and Hard limit event rates can be used as a tool for assessing drug library update effectiveness when analyzed over time and for different profiles. 
Relative trends of compliance and Hard limit events were observed, as well as changes after drug library updates. Relative trends of compliance and Hard limit events followed by a non-compliant (non-PG) infusion were also observed. Comparison of trends of compliance, Hard limit events, and Hard limit events followed by a non-PG infusion was a medium for hypothesis synthesis and future research directions. Observing the trends can help in discovering effects of drug library updates to determine whether the library should be revised due to high Hard limit rates or if it is helping to reduce Hard limit events along with increasing compliance. A decrease of Hard limit events combined with a decrease of compliance is an indicator that the drug library is being bypassed and is a practice that shows the smart pump is not being used as intended. Future work in this area may include applying similar analysis over a period of two years or more to find any seasonal trends, and applying the monthly analysis for only one specific profile.

\section{Discovered potential human-computer interaction issue with regards to drug labels and nomenclature in drug library}

In this case study, Hard limit events for the gentamicin drug in the general pediatrics and surgery department were identified. Events following each Hard limit event were analyzed to find what follow-up actions were taken afterwards. The follow-up actions were used to classify events into two types of user errors: programming (order entry) errors and selection errors (errors where the wrong gentamicin drug option was selected). Results showed that more than $50 \%$ of Hard limit events were caused by a selection error. One of the follow-up actions after a selection error is the setup of a non-PharmGuard infusion. The gentamicin case study is a representative case sample of how 
factors of human-computer interaction, such as infusion pump design and drug library labels, are important factors in infusion pump usage influencing compliance and Hard limit events. Specifically, this research showed the value of a smart pump system by identifying order entry errors, and identifying a new type of user error that increased smart pump Hard limit events from drug library usage: drug selection error.

\section{Described a decision tree method for infusion pump data analysis.} The method was applied to identify factors that have a stronger association with infusions that hit a Hard limit event than infusions that are not associated with a Hard limit event.

A set of infusion data was created with two classes: one class that was associated with a Hard limit event and one class that was not. The data set was used to build a decision tree. Factors that had a split that separated $66 \%$ or more of the infusion cases associated with a Hard limit event were identified as indicators that favour Hard limit events. The first split in the decision tree, identified two units at CHEO, the emergency department and the neonatal intensive care unit (NICU). The two departments had twice as many Hard limit events as other departments for the data set of infusions tested. A literature review of medical error and patient safety research in these two departments confirmed that these units have potential for all types of clinical errors as both areas have very specific challenges in providing patient care. A previous study on prescription errors at $\mathrm{CHEO}$ showed that the NICU had the highest rate of prescription discrepancies. The decision tree technique results suggest that Hard limit events are more common in these areas, which could be an indicator that other medical errors found in previous research could be also present in these units and that further research and attention should be paid for those 
areas. The second significant factor identified was patient weight: a weight over $48 \mathrm{~kg}$ had double the Hard limit event rate than infusions with a weight

below $48 \mathrm{~kg}$. Decision tree analysis could be applied using different types of smart pump data (e.g., alarms), and not just infusion data.

The work on the gentamicin case study (contribution 3) was disseminated in the publication: A case study: gentamicin hard limit events and follow-up actions in smart infusion pumps in the Canadian Medical and Biological Engineering Conference (CMBEC) in May 2013 [5].

\subsection{Thesis organization}

Chapter 2 in this thesis presents background information on clinical errors, and infusion pumps; the research findings on medication errors associated with infusion pumps; and current research on smart infusion pumps and drug libraries. Chapter 3 provides an overview of CHEO in terms of its facilities, services, patient population, and the process of medication prescriptions from doctor to patient. This chapter also describes the Medfusion 4000, its operation, the drug library, the stakeholders, and the results from the analysis of compliance and Hard limit event trends. Chapter 4 presents a case study done to analyze follow-up actions taken by clinicians after a Hard limit event during a gentamicin drug delivery in one of CHEO's departments that has a high Hard limit event rate for the drug. Chapter 5 describes the decision tree technique, and applies it to a known data set for testing the technique and assessing the validity of the information presented in the tree. Chapter 6 applies the decision tree technique to infusion pump data to find prominent factors differentiating between infusions that hit a Hard limit event and those that do not. Chapter 7 is the conclusion chapter; it concludes our research findings and recommends future work. 


\section{Chapter 2}

\section{Background}

This chapter is divided into two major parts. The first part, 2.1, presents a literature review on medical errors and infusion pump errors. The second part of this chapter, 2.2, describes the data mining technique applied in this thesis.

\subsection{Introduction to medical errors}

The Institute of Medicine raised public awareness on errors in health care through the publication of two widely cited reports: To Err is Human: Building a Safer Health System in 1999 [6] and Crossing the Quality Chasm - A New Health System for the 21st Century in 2001 [7]. There have been numerous studies researching the occurrence and causes of medical errors. To name a few recent studies: research on intravenous medication errors found $69.7 \%(396 / 568)$ of intravenous administrations to have at least one clinical error, $25 \%$ of which were serious and likely to cause harm [8]; research on errors in long-term residential care found that older patients were more prone to errors [9]; research in a pediatrics emergency department investigating incident reports found that $19 \%$ (597) of reports corresponded to medication errors from a yearlong study [10]. A recent study was conducted by the collaboration of the Office 
of Chief Medical examiners or Chief Coroners of four provinces with the Institute of Safe Medication Practices (ISMP) Canada to identify deaths caused by medication incidents [1]. A total of 523 death reports between 2007 and 2012 that were suspected to be caused by a potential medication incident were reviewed. The review team made up of three pharmacists, one registered nurse, and a physician experienced as a coroner determined that 115 of the medication incidents were incidents that contributed to or resulted in a patient's death. System and human errors in the health care system place an unwanted risk in the health care environment, compromising patient safety, a fundamental element of high quality health care [11]. Medical errors also incur additional unwanted costs to the health care system. In the case where an error affects a patient negatively, there is potential of requiring additional care and extending the patient's length of stay at the hospital. Based on the Agency of Healthcare Research and Quality (AHRQ), an accidental overdose costs on average $\$ 6000$ per incident [12]. A cost study done by the Journal of the American Medical Association (JAMA), estimated that a preventable adverse drug event caused by errors costs $\$ 4585$ and results in an extra 4.6 days in hospital on average [13]. Through research, an increased understanding of issues leading to errors is gained and aids in reducing and eliminating errors with the aim to improve patient safety and reduce health care costs. Medical errors can be categorized in the following manner [14]:

\section{- Diagnostic error:}

- Error or delay in diagnosis

- Failure to employ indicated tests

- Use of outmoded tests or therapy

- Failure to act on results of monitoring or testing

\section{- Treatment error:}


- Error in the performance of an operation, procedure, or test

- Error in administering the treatment

- Error in the dose or method of using a drug

- Avoidable delay in treatment or in responding to an abnormal test

- Inappropriate (not indicated) care

\section{- Prevention error:}

- Failure to provide prophylactic treatment

- Inadequate monitoring or follow-up of treatment

\section{- Other error:}

- Failure of communication

- Equipment failure

- Other system failure

Medication errors, which represent a large portion of medical errors, can occur at any of the following medication administration stages [6]:

- Ordering stage: The stage where the nurse or doctor decides on the medication treatment prescribed to the patient. Possible errors in this stage are wrong dosage, or wrong choice of drug.

- Transcribing stage: The stage where the nurse or doctor transcribes the ordered drug, and necessary information (e.g., dose, frequency, rate). Possible errors in this stage are incorrect frequency of drug administration, or missed dose because a medication is not transcribed. 
- Dispensing stage: The stage where drug is retrieved and formulated if need be (e.g., in proper concentration). Possible errors are when the drug is not sent in time to be administered at the time ordered, wrong drug retrieved, wrong dose collected by pharmacist.

- Administering stage: The stage where the medication is delivered to the patient in appropriate form and via appropriate methods. Possible errors are also wrong dose of drug administered, and wrong technique used to administer the drug.

- Monitoring stage: The stage where the patient is monitored for expected therapeutic effects and side effects. Possible error is not noting the effects of the given medication.

As a device used for medication administration, infusion pump errors fall under errors in the administering stage. Infusion pumps are described in detail in the next section.

\subsubsection{Infusion pumps}

Medications in liquid form can be delivered in a controlled manner to patients with the use of infusions pumps [15]. An infusion pump is a medical device designed to enable programming to automatically administer set amounts of liquids into a patient over a specific period of time [16]. A basic infusion pump has three main components (Figure 2.1):

1. a fluid reservoir, a container holding the liquid (e.g. syringe or bag) and connected to the pump; 


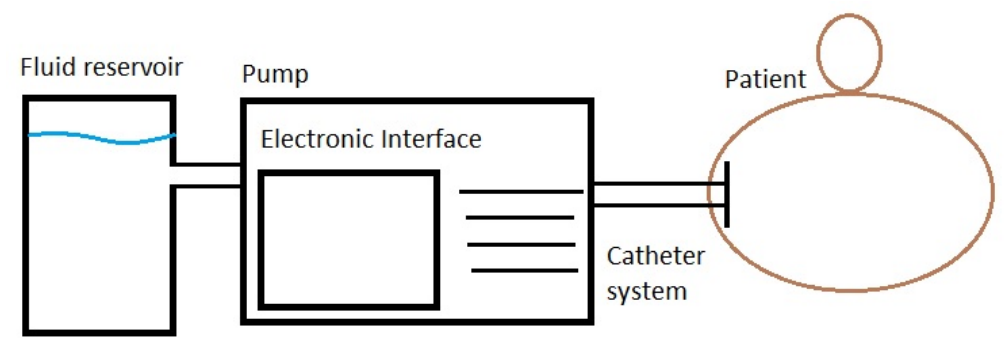

Figure 2.1: Basic infusion pump and components: 1) fluid reservoir, 2) catheter system, and 3) electronic interface.

2. a catheter system for transferring the liquids from the reservoir to the patient, and

3. an electronic interface, to generate and regulate flow, controlled by a humancomputer interface for programming the rate, infusion time or dose of the prescribed drug [16].

Problems with infusion pumps related to insertion of the catheter system are a major issue with the use of a pump; however, there are several benefits for its use (e.g., allows for continuous infusion over long periods of time, maintains constant blood levels) $[17,18]$.

When research into medical errors became a growing field in health care and studies found errors related to human-computer interaction issues with medical device use, infusion pump manufacturers began designing infusion pumps able to detect errors in programming/setting infusion pumps. More recent infusion pumps, called smart pumps, guide users in programming the pump based on drug libraries. A drug library is a customizable list of drugs with limits on dosage, delivery time and/or delivery rates for every drug. A drug library specifies drug dose, time and rate ranges that cannot be exceeded (hard limits) and ranges that are not recommended for normal use but may be administered if the user confirms the entered infusion values 
and overrides the alert (soft limits). In addition, all smart pumps have an event log or database where all events or actions occurring on the pump are recorded. Some examples of recorded events are:

- beginning of an infusion

- end of an infusion

- pump turned on

- pump turned off

- mechanical alarms

- change of drug library

- hard limit exceeded

- soft limit exceeded

The event/history log provides details on how the pump was programmed, relevant alarms, and infusion delivery status which allows retrospective analysis on any pump related issue and activity.

Most smart pumps give the option of setting or programming an infusion outside of the library. Namely, the infusion is set up by entering a combination of dose, time and rate. When an infusion is programmed outside of the drug library, errors cannot be detected. A well designed drug library will have several infusion programming steps to discourage bypassing the drug library. If the option to bypass a pump is permitted too early, then it would be too easy to bypass the drug library when it is not necessary to do so. Compliance is the use of smart pumps with the drug library and is presented as a percentage of the number of infusions setup in the drug library 
over the total number of infusions. A high compliance indicates high usage of the drug library when setting up an infusion. There may be clinically valid reasons for setting an infusion outside of the library (e.g., library does not include the drug or special patient requiring doses outside of the library range).

A study on nurses' experience with pump implementation, use, and acceptance revealed overall positive feedback on acceptance of the technology [19] .

\subsubsection{Medication errors and infusion pumps}

Research with direct evidence linking medication errors to infusion pump programming is difficult to find for infusion pumps with no event log or tracking databases. Most of the research available on this topic is performed on smart pumps; however, two organizations, the Institute for Safe Medication Practices (ISMP) Canada, an independent national non-profit organization [20], and MEDMARX, an American adverse drug event reporting registry [21], did publish research using voluntary medication error reports that linked medication errors with infusion pumps. In a study done in partnership with the Canadian Association of Paediatric Health Centres (CAPHC), 11 CAPHC member organizations submitted records to ISMP on medication incidents from October 2005 to June 2008 [22] . The purpose of the study was to analyze the medication incidents of the top five drugs found in medication incident reports in paediatric patients. A total of 320 medications were found in the 294 incidents examined (11 reports involving either adverse drug reactions or unclear information were excluded). After the five most frequently found drugs were identified (morphine, potassium chloride, insulin, fentanyl, and salbutamol), their respective qualitative incident reports were further examined. In three of the 176 reports on morphine, pump programming was a factor, where the wrong units were entered. For insulin, when infusion problems were involved, the main factor was IV line mix-up. Several of the 
30 fentanyl reports were overdoses involving pump programming. The main finding from the CAPHC study is that infusion pump programming is a factor in medication incidents involved in the top five drugs in pediatrics, which emphasizes the importance of research on infusion pumps.

MEDMARX is an adverse drug event reporting registry that is the largest in the U.S. with over 400 health care facility members [21]. Its goal is to provide resources to address errors and adverse drug events, to mitigate strategies and interventions to prevent errors and better understand them. In a study performing a secondary analysis on MEDMARX data for errors reported by the PACU (post-anesthetic care unit) between the period of August 1998-March 2002, there were 32/645 reports caused by improper use of the infusion pump [23]. Case reports show decimal error discrepancies in flow or rate inputs, which suggests errors were directly related to infusion pump programming.

The Food and Drug Administration (FDA) received nearly 56000 incident reports on infusion pumps between 2005 and 2009, the highest number of reports for one medical device [15]. Errors of all types were reported in incidents: human factors issues, alarm errors, and software errors.

\subsubsection{Medication errors and smart pumps}

Several studies have been conducted on smart pumps to evaluate smart pump impact and benefit of technology; namely, to prove the reduction of medication errors associated with infusion pumps. Research conducted by Sandlin et al. show that errors associated with pediatric medications are a significant cause of preventable adverse events in the health care arena, and smart infusion pumps with drug libraries are recommended for reducing the amount of errors [24]. 
A study from October 2003 to January 2005 observed smart pump data for anticoagulants and found that a total of 863 alerts were generated for $7.3 \%$ (268) of the 3,674 patients [25]. Of the 863 library alerts for anticoagulants, $43.1 \%$ resulted in reprograms, $46.5 \%$ were cancelled and $10.4 \%$ were continued. Further examination of the 372 reprograms revealed that the library alerts prevented 10-fold potential overdoses in 40 patients $(28.5 \%)$, 100-fold potential overdoses in 40 patients $(28.5 \%)$, and $>100$-fold potential overdoses in 10 patients (6.5\%). One-hundred-fold potential under-doses were intercepted in 39 patients $(25.2 \%)$, and 10-fold potential underdoses were intercepted in 20 patients $(16.8 \%)$. Another study revealing positive results; showed that there were 494 critical catches within the 9-month study period. The study, however, did not reveal any details on errors, and whether the pump was linked to patients or prescriptions. It is unsure whether or not errors were due to prescriptions or order entry [26]. A study over a 5-month period using data from 14 US pediatric hospitals assessed smart pump error prevention rate and $83 \%$ of alerts that were overridden, $10 \%$ were re-programmed and $14 \%$ were cancelled [27]. Two studies ( $[28,29])$ investigating smart pumps conclude that smart pumps are efficient in detecting programming errors; however low compliance is a prevalent issue in the use of drug libraries which ensure patient safety. In [29], researchers implemented an IV infusion safety initiative which included smart pump implementation as part of the new system (along with other technologies, standardizations, and a culture of safety) that allowed detection of input errors and tracking of infusion pump use which led to an overall safer environment.

In a case study of a medication error incident at a facility using smart pumps, an analysis revealed that the error was caused by library bypass; that is, non-compliance to the library, and that the library would have prevented the incident [30]. The case led to a prospective initiative and study on improving compliance at the institution. 
The study revealed that education on the purpose of smart pumps and risks associated with not using one was a factor in improving compliance from $28 \%$ to $85 \%$ over the study period. The study also included other steps taken to improve compliance and suggestions. User awareness of the benefit of technology, specifically positive impact of drug library, can motivate clinicians to comply to the smart pump.

Certain studies however, reveal findings showing that drug libraries only eliminate a small portion of infusion pump errors. A study by Husch et al. compared medication drug, dose, rate and patient in the prescription and recorded in the infusion pump event logs. Results found that 3 of the 389 errors appeared to be order entry (e.g. typo errors). 37 of the 389 errors detected had 1) a different rate in the prescription and in the infusion log, 2) a different weight, or 3) an incorrectly calculated weight-based dose. Further analysis of those 37 cases showed that only 1 of the 389 errors would have been caught by smart pump technology because it was the only case where the deviation was outside of the standard limits [31]. The conclusion to these findings is that though drug libraries are necessary for patient safety and avoiding serious problems, they only eliminate a small portion of infusion pump errors; therefore they are only a part of the solution. The study found that common errors were in the ordering stage (order errors), dispensing stage (labelling errors) and administration stages (patient identification); programming errors were not among the common errors. A study by Nuckols et al. compared preventable adverse drug events before and after smart pump implementation found that 4 out of 100 errors identified were detectable by the drug library [32]. The library did not have limits appropriate for bolus type infusions (infusions given over a short period of time in relatively larger dosages), dosing ranges were too wide for certain patients and monitoring problems were more common than administration problems. Suggestions made by Nuckols et 
al. on improving smart pumps are interfacing with electronic medical records, computerized prescriber order entry, bar coded administration systems, and pharmacy information systems.

\section{Example 1: Infusion pump error}

This example is from a recent article from the Ontario Critical Incident Learning journal issued on February 2014 [33]. In this case, a smart infusion pump was used to set up a propofol prescription with a concentration of $1000 \mathrm{mg} / \mathrm{mL}$ for a critically ill patient. The drug library did not have a standard concentration for propofol; therefore, the nurse had to enter the concentration manually, outside of the drug library. The nurse entered $10 \mathrm{mg} / \mathrm{mL}$, which was the wrong concentration by 100fold. The error was only noticed after the entire propofol bottle was administered in only 20 minutes; the patient died as a result of the overdose. This example is a case that supports the use of drug libraries and standard concentration and dosing practices. Other factors contributing to the error was that the propofol bottle was purchased from a manufacturer that labelled their bottles differently than the usual manufacturer (e.g., drug information in different locations on the label), and that the bottle hangs upside down when connected to the pump, making the label harder to read. This example also shows how a series of minor events can lead to an error.

\section{Example 2: Smart infusion pump error}

This example is from an incident reported to the Food and Drug Administration [34]. In this case, the nurse was setting up an infusion for parenteral nutrition on a smart pump that displayed a list of rates as options. The nurse selected $457 \mathrm{~mL} / \mathrm{hr}$ instead of $45.7 \mathrm{~mL} / \mathrm{hr}$, a ten-fold error. This value generated a soft limit alarm which the nurse overrode. The error was noticed two hours later and the patient was sent to 
the intensive care unit for suffering from acute hyperglycemia. This is an example of issues with drug library limits, and smart pump design.

\subsubsection{Drug library research}

Developing the initial deployed drug library and updating the drug library are crucial for having an effective smart pump system. Hospital researchers have published papers on their process for developing drug libraries which centers newly adopting this technology can benefit from [35]. Alaris Medical Systems, Inc., a developer of smart pumps, sponsored in August 2002 for its first smart pump the creation of a starter drug library. By October 2002 it was evident that the drug library needed to be modified by different hospitals to meet the various practices in each institute [36]. Manrique-Rodriguez et al. also published a paper on alarm analysis to assess the practical significance of alarms and implications towards drug library and hospital protocols [37]. In their paper, data were analyzed over four months after the deployment of their first library (first version) in the pediatric intensive care unit. The analysis identified inconsistencies between library limits and practice at the hospital, and allowed them to modify accordingly. A key to ensuring that library and practice are in line is communication between pump users (clinicians), clinical engineers and the pharmacist responsible for the drug library [38].

Researchers studied the interface of a smart pump and steps taken to program the infusion pump [39]. The study stated the complexity of the interface was due to the limited number of keys that served more than one function depending on the state of the pump. For several tasks, subjects entered unnecessary steps to complete the task. The study did not find a correlation between experience with the pump and the percent of goal oriented strokes taken towards completing the required task, suggesting that the interface was too complex for users to learn with experience alone. 
Additionally, the extra unnecessary steps taken to complete an infusion confirmed in this study are the reason why it is complex to analyze events from infusion logs directly or automatically.

\subsubsection{Proposed solutions for reducing infusion pump errors in research}

One proposed solution is a virtual infusion pump training module proposed, implemented and tested in [40]. The training module could be used to test the pump design, test the drug library updates, and be used for training. A study measured improvements in a nurse's ability to administer medication to pediatric patients with and without intense simulation training, and showed that large improvements were made with this form of training [41].

Computerized provider order entry (CPOE) is a technological alternative to handwritten orders where the prescription is completed in an electronic form with fields that perform automatic calculations, and give standard concentration or dose options [42]. This technology has the potential to reduce prescription errors and infusion pump errors caused by misreading prescriptions and dose miscalculations.

\subsubsection{Regulatory activity}

Research in infusion pumps and associated errors has led to the start of several initiatives towards creating standards and guidelines for manufacturers and hospitals on infusion pump systems. In 2010, the FDA started the "infusion pump improvement initiative" as a response to the large number of reported incidents [43]. The FDA's initiative has several components, starting with the creation of the FDA infusion pump website in order to raise awareness on the infusion pump problems. In April 
2010, a guideline document for pump manufacturers on additional information to be added in premarket submission reports, and a letter sent to manufacturers describing the scope of the problem and the general types of errors being reported for considerations in redesigns. In May 2010, an infusion pump workshop was held gathering stakeholders to discuss the issues, future work, and the improvement of regulatory process among other things. The FDA started a Generic Pump Project in collaboration with researchers from the University of Pennsylvania in Philadelphia and the Fraunhofer Center for Experimental Software Engineering in College Park, MD, to design an infusion pump model and reference specifications for designing safe pump software, and software testing.

In October 2010, the Association for the Advancement of Medical Instrumentation (AAMI) and the FDA held an infusion device summit, inviting manufacturers, researchers, pharmacists, doctors and nurses to create a list of priority issues for improving new infusion pumps systems [44]. Among the priority issues focused on were user error mitigation; standardization of processes for reporting, aggregating, and analyzing infusion pump incidents; and integration of infusion pumps with information systems and drug libraries.

In 2009, ISMP held an infusion device summit for developing guidelines on safe use and implementation of smart infusion pumps. The smart pump infusion pump system is different than the previous infusion pump technology, requiring the involvement of new departments (e.g., information technology or clinical informatics) and a commitment to a medication safety program that may necessitate cultural change in the hospital in order to succeed. Smart pumps work effectively only with the use of drug libraries, which need to be designed using standard medication dosing; a lack of dosing standards will limit the use of the drug library and therefore, smart pumps would not be used to its full advantage. 
In conclusion, the development and implementation of smart infusion pump technology is progressing in the right direction towards improving patient safety and reducing mediation errors. There are many opportunities for technology advancement in the smart pump industry for manufacturers, researchers and clinical engineers.

\section{$2.2 \quad$ Introduction to data mining}

Data mining techniques are used to gain knowledge in order to predict outcomes, discover associations and reveal patterns not obvious to the human observer [45]. Mining techniques are especially useful for large data sets where it is a challenge to process and extract useful data. In the health care field, data mining techniques are being applied to medical data in order to gain knowledge and information that is different from information gained from traditional data analysis techniques- statistics or observational analyses- and that can be more meaningful.

A study compared four data mining techniques to predict patient outcome from coronary surgery to provide better patient management [46]. In one study, association mining was used to replace unknown parameters in patient problem lists found in electronic health records where missing data are commonly found [47]. The techniques decision trees and neural networks were applied to improve diagnosis of skin diseases, including skin cancer [48]. Clustering and association mining methods were also used for gaining knowledge about HIV/AIDS to improve prevention and treatment management [49]. Prediction models were applied to schedule emergency room physicians based on previous data [50]. Haemodialysis patient outcome was predicted using mining techniques to reduce hospitalization [51]. These works are only a small set of examples of data mining applied to medical data.

A data mining problem formulation requires a data set with one or more variables, 
called a feature vector, to represent each unique case or sample in the data set. The selection of the data mining technique is made based on the research question and the available data. Knowledge of the data types of the variables in the data set is needed for selecting a suitable data mining technique. Different techniques will give more meaningful results for data sets with specific data types. A large group of data mining techniques are designed for data classification, which are used for categorizing, and grouping the samples in the data set.

Current studies analyzing smart pump data apply manual data analysis techniques requiring the researchers to acquire knowledge by manually filtering through large amounts of data. In a literature review of smart pump research no papers were found to be published on the application of data mining techniques to infusion pump data. The data mining technique used for analyzing the infusion pump data in this thesis is the decision tree method, a popular classification technique. The decision tree technique creates a set of rules using variables in the feature vector that are used for classifying the data. Decision trees can be used in many ways to examine a data set, the most popular being to determine the class of a sample/case or object where the class was previously unknown. Decision trees are also applied in research to identify factors influencing data classification and differentiation, and this is how decision trees will be used in this thesis. The interest of this part of the research will be to investigate the use of decision trees in identifying the factors that differentiate between different infusion types; rather than classifying the data, making a decision or discovering the rules. These factors identified may reveal patterns that can be used to make a decision on how to improve the drug libraries, or target training. The factors available are the infusion parameter variables extracted from the PharmGuard reports and organized into the feature vector.

Decision trees have been used in several applications to identify factors influencing 
data classification and differentiation. A study identified factors for classifying types of car accidents (based on severity) that could be used to implement priority actions based on accident type [52]. A study used decision trees to classify eye data taken before laser eye surgery to ultimately find if a condition occurring after surgery can be predicted before the surgery by looking at the tree's classification factors [53]. To assess decision trees in discovering patterns in the infusion data and to validate meaning and interpretation of infusion data results, decision trees are firstly applied to a mushroom data set; a smaller data set described by categorical (non-numerical) data, similar to the infusion data, that has known rules. The mushroom data set is described in the next section.

\subsubsection{Mushroom data}

The mushroom data set is a publicly available dataset from the Stanford data mining repository [54]. The dataset is made up of 8124 samples of data with 21 categorical variables (see Table 2.1) that divide the data into two classes: 3916 poisonous or 4208 edible. At the time of public availability of the mushroom data online in 1997, the simplest set of classification rules consisted of a series of four logical rules using six attributes that correctly classify $100 \%$ of the data:

Table 2.1: List of 21 variables and possible values for mushroom data set.

\begin{tabular}{|c|l|}
\hline Attributes & Possible Values \\
\hline \hline cap shape & bell, conical, complex, flat, knobbed, sunken \\
\hline cap surface & fibrous, grooves, scaly, smooth \\
\hline \multicolumn{2}{c}{ Continued on next page } \\
\hline
\end{tabular}


Table 2.1 - continued from previous page

\begin{tabular}{|c|c|}
\hline Attributes & Possible Values \\
\hline cap color & $\begin{array}{l}\text { brown, buff, cinnamon, gray, green, pink, purple, red } \\
\text { white, yellow }\end{array}$ \\
\hline bruises & yes, no \\
\hline odor & almond, anise, creosote, fishy, foul, musty, none, pungent \\
\hline gill attachment & attached, descending, free, notched \\
\hline gill spacing & close, crowded, distant \\
\hline gill size & broad, narrow \\
\hline gill color & $\begin{array}{l}\text { black, brown, buff, chocolate, gray, green, orange, pink } \\
\text { purple, red, white, yellow }\end{array}$ \\
\hline stalk shape & enlarging, tapering \\
\hline stalk root & bulbous, club, cup equal, rhizmorphs, rooted, missing \\
\hline stalk surface above ring & fibrous, scaly, silky, smooth \\
\hline stalk surface below ring & fibrous, scaly, silky, smooth \\
\hline stalk color above ring & $\begin{array}{l}\text { brown, buff, cinnamon, gray, orange, pink, red, white } \\
\text { yellow }\end{array}$ \\
\hline & Continued on next page \\
\hline
\end{tabular}


Table 2.1 - continued from previous page

\begin{tabular}{|c|l|}
\hline \multicolumn{1}{|c|}{ Attributes } & Possible Values \\
\hline \hline stalk color below ring & brown, buff, cinnamon, gray, orange, pink, red, white \\
& yellow \\
\hline veil type & partial, universal \\
\hline veil color & brown, orange, white, yellow \\
\hline ring number & one, two, none \\
\hline ring type & cobwebby, evanescent, flaring, large, none, pendant \\
& sheathing, zone \\
\hline spore print color & black, brown, buff, chocolate, green, orange, purple, white \\
& yellow \\
\hline population & abundant, clustered, numerous, scattered, several, solitary, \\
\hline habitat & grasses, leaves, meadows, paths, urban, waste, woods \\
\hline \hline
\end{tabular}

A mushroom is poisonous:

1. if odor $=\operatorname{NOT}($ almond or anise or none), then poisonous. 120 poisonous cases missed.

2. else if spore-print-color $=$ green, then poisonous. 48 poisonous cases remaining. 
3. else if odor $=$ none AND stalk-surface-below-ring = scaly AND stalk-colorabove-ring $=$ NOT brown, then poisonous. 8 poisonous cases missed.

4. else if (habitat $=$ leaves $\mathrm{OR}$ population $=$ clustered $)$ AND cap-color $=$ white, then poisonous.

A mushroom that does not meet the criteria above is not poisonous.

Over $98 \%$ of poisonous mushrooms can be classified by excluding mushrooms with an almond odor, anise odor or no odor at all. From this, we can conclude that odor is a factor with a large influence in determining whether a mushroom is poisonous or edible. The decision tree facilitates this discovery as important factors (such as odor) appear at the top of the tree.

\subsubsection{Decision tree methodology}

A decision tree is a structure that splits a dataset into smaller and smaller subsets, based on the value of one or more variables at every split, until the final subsets of data are grouped by class $[55,56]$. Figure 2.2 is an example of a decision tree created by the mushroom data classification rules. The decision tree technique used to create a decision tree begins with a root node where all the cases are assigned. From there, a recursive algorithm is applied to the root node and to each newly created nodes. A parent node is any node that is divided into two or more nodes that are called children nodes. Leaf nodes are the final nodes that are not divided and are assigned a class label: poisonous or edible in the case of the mushroom data set. All nodes are children nodes, except for the root node, and all nodes are parent nodes except for the leaf nodes. If a random mushroom from the data set is selected, we can determine if it is a poisonous or edible mushroom by starting from the top at the root node and moving down the tree by comparing the values in the mushroom's feature vector to 


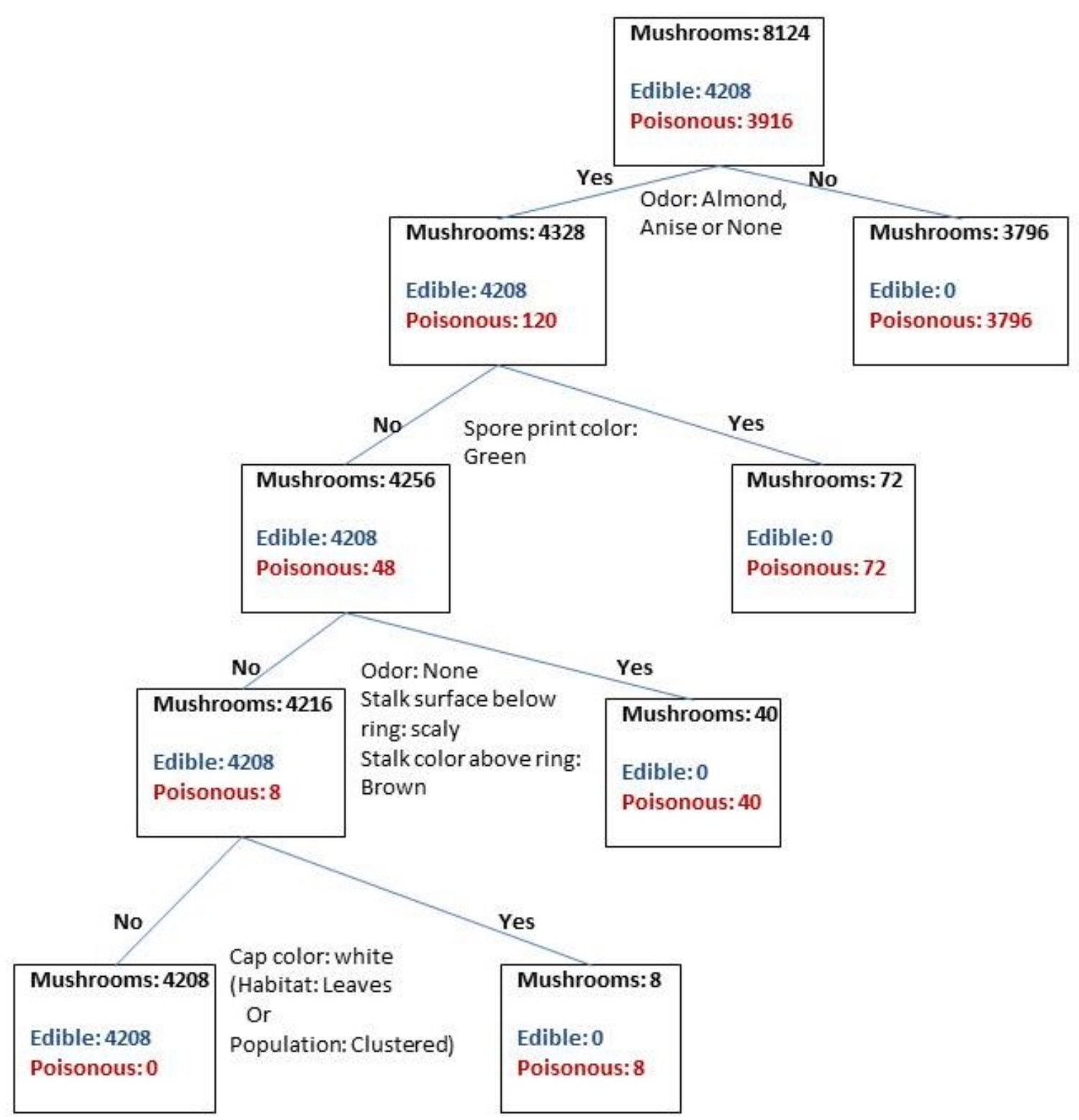

Figure 2.2: Classification rules for classifying poisonous and edible mushrooms presented in chart form. 
the variable(s) at every split. If the mushroom sample reaches a leaf node that is labelled poisonous, then the mushroom is poisonous, if it reaches a leaf node that is labelled edible, then the mushroom is edible. After the initial tree is built, the tree can be used to classify new mushrooms that are not in the original data set to be poisonous or edible.

There are a few different types of decision trees and decision tree algorithms for building the trees; only the method used for this thesis is described in this section. A univariate binary decision tree algorithm splits each node into only two nodes based on one variable only [56]. To create the tree, starting at the root node, for each variable, impurity (2.1) for the children nodes is computed for every possible split, and the split with the lowest impurity measure is chosen. The ideal split is a split that creates pure children nodes, nodes that have samples of only one class, and the impurity is a measure of how close the nodes are to being pure [55,57]. The lower the impurity measure, the closer the nodes are to being pure. In information gain theory, the split with the lowest impurity measure will have the highest information gain when comparing the impurity measure of the children nodes to that of the current node being tested based on Eqn.2.2, where Impurity(Parent) is the impurity of the current node being split (future parent) and Impurity(Children) is the average impurity measure of the two future children (from Eqn.2.1). Normally, when testing different splits, the Impurity(Parent) factor is a constant; therefore, only the Impurity(Children) factor is computed and compared. For testing splits of countinous numerical variables, all possible variable values (based on the data set) are listed in order, and a split is tested halfway between any two adjacent unique values. The set of possible values that a categorical variable can hold are called levels in some literature. For example, colour is a categorical variable and two possible levels are blue and red. For a categorical variable with $L$ levels, there are $2^{L-1}-1$ possible 
binary splits. $L$ values can be assigned to the left and right nodes in $2^{L}$ ways: for the color example, the possible assignment options are presented in Figure 2.3. Because the split is a binary split, two of the possible splits will have an empty child node; therefore two splits are always eliminated. The remaining possible splits are found in identical pairs with the left and right children nodes switched; therefore, the final number of splits is divided by two.
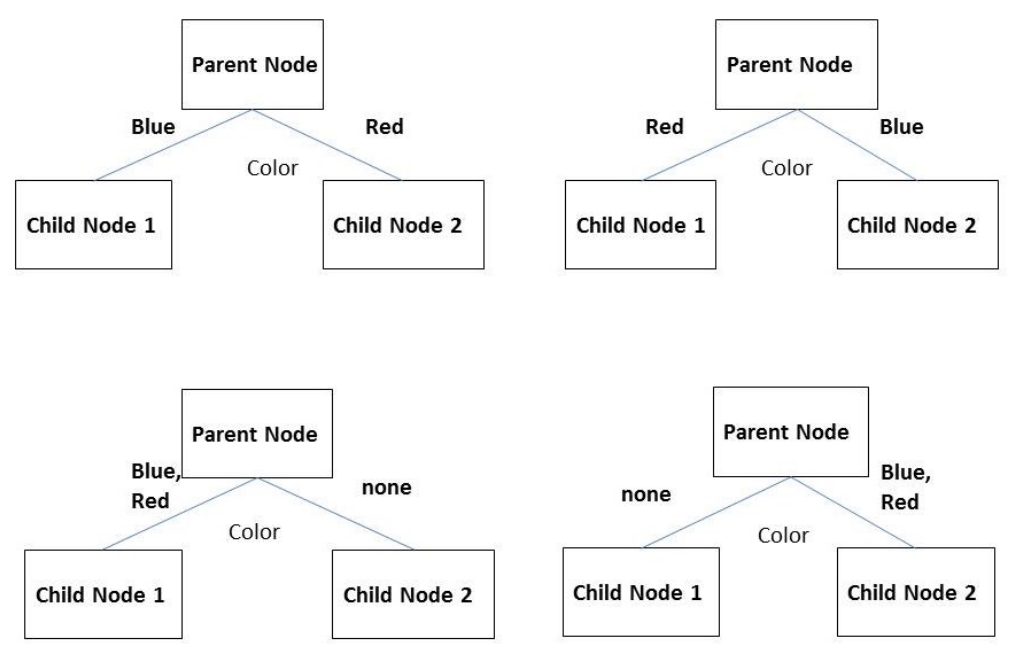

Figure 2.3: All the possible splits for a categorical color variable with level values of blue and red

\section{Average impurity measure of a split made of two children nodes:}

$$
\operatorname{Impurity}(\text { Children })=F_{L} \times I_{L}+F_{R} \times I_{R}
$$

where $F_{L}$ is the fraction of data assigned from the parent node to the left node, $F_{R}$ is the fraction of data assigned from the parent node to the right node, $I_{L}$ is the impurity measure from the left node, and $I_{R}$ is the impurity measure from the right node. 


\section{Information gain of a split:}

$$
\text { InformationGain }=\text { Impurity }(\text { Parent })-\operatorname{Impurity}(\text { Children })
$$

\subsubsection{Split criterion}

The split criterion is the decision tree element used to define the optimal split for each node. The optimal split may vary based on the split criteria; therefore, different criteria may produce slightly different trees. The most informative tree based on split criteria is dependent on application because different trees may use different factors in determining final data classification.

Two common split criteria are: 1) gini (2.3), and 2) deviance (2.4), also referred to as entropy. Both gini and deviance are impurity measures that quantify the impurity created by a node. For a pure node, the gini and deviance criteria are both equal to zero, otherwise, both are equal to a positive integer. The gini criterion seeks a split which increases the purity of one child node even if the number of cases in the children nodes are imbalanced; on the other hand, the deviance criteria seeks a split which will also balance the children nodes more or less [58]. For a data set with imbalanced classes, the gini index is the preferred criteria because the children nodes will not need to be balanced.

\section{Gini Split Criteria}

$$
I_{X}=1-X_{1}^{2}-X_{2}^{2}
$$

and 


\section{Deviance Split Criteria:}

$$
I_{X}=-X_{1} \times \log \left(X_{1}\right)-X_{2} \times \log \left(X_{2}\right)
$$

where, $X_{1}$ is the fraction of class 1 in node $X, X_{2}=$ fraction of class 2 in node $X$. For the left node $X=L$ and for the right node $X=R$.

Figure 2.4 represents the gini and deviance curves for different fractions of cases belonging to one class. The plots are for a two class data set. For both plots, the impurity measure is 0 when the node is pure (has only class 1 cases, at $\mathrm{x}=0$ or has only class 2 cases at $\mathrm{x}=1$ ), and positive when the node is not pure. The figure shows that the value for the deviance criteria is always greater than the gini criteria for the same proportion of class 1 to class 2 cases; therefore, the difference in the impurity measure using both criteria will be affected by the proportion of cases in the children nodes $F_{L}$ and $F_{R}$.

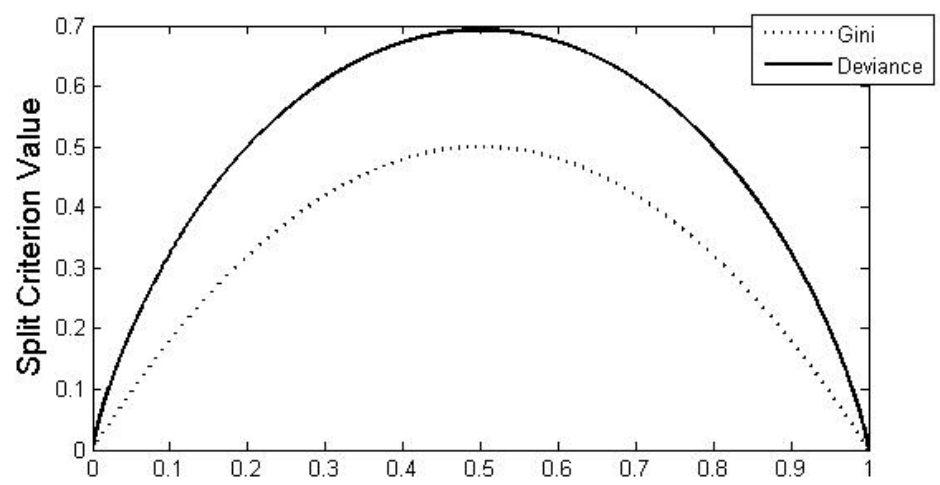

Figure 2.4: Gini and deviance curves for different fractions of $X_{1}$

The following example will demonstrate the difference between the two split criteria (or impurity measures) in a situation where there is a clear dominance of one class in the sample set (i.e., class imbalance); the gini split criterion will 'favour' a split 
that will lead to a pure node for the minority class despite having fewer cases in the child node, while the deviance criterion will favour a split that appears to maximize the number of cases isolated from the minority class despite errors in misclassifying the dominant class. In the first tree (a) of Figure 2.5, the predictor with values 1 and 9 are highlighted, while only value 9 is highlighted in the second tree (b).

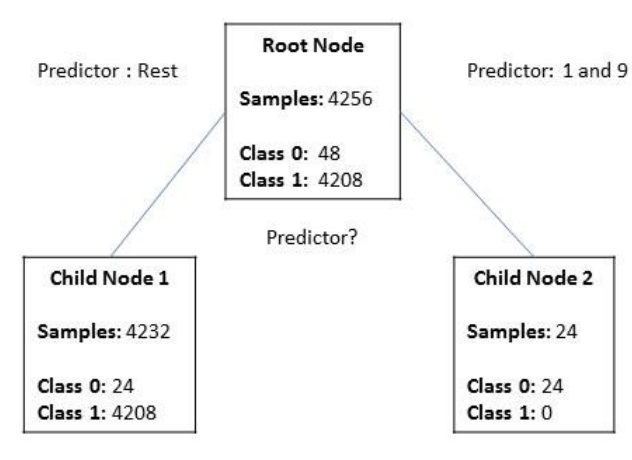

(a) Gini criterion

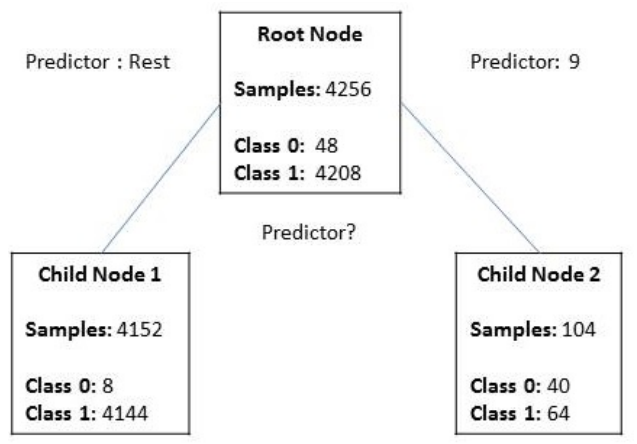

(b) Deviance criterion

Figure 2.5: Decision tree splits with gini criterion (a) and deviance criterion (b)

Sample calculations for calculating the Impurity for the trees in Figure 2.5 are shown for both the gini and deviance criteria. Recall that impurity(Parent) is a constant; therefore, only impurity(Children) need to be calculated to simplify the steps.

Calculation of impurity using the gini criterion:

$$
\begin{aligned}
& I=F_{L} \times I_{L}+F_{R} \times I_{R} \\
& =\left(\frac{4232}{4256}\right)\left(1-\left(\frac{4208}{4232}\right) \times\left(\frac{4208}{4232}\right)-\left(\frac{24}{4232}\right) \times\left(\frac{24}{4232}\right)\right)+ \\
& \left(\frac{24}{4256}\right)\left(1-\left(\frac{0}{24}\right) \times\left(\frac{0}{24}\right)-\left(\frac{24}{24}\right) \times\left(\frac{24}{24}\right)\right) \\
& =0.994 \times(0.0112)+0.0056 \times(0) \\
& =0.0112
\end{aligned}
$$


Calculation of impurity using the deviance criterion:

$$
\begin{aligned}
& I=F_{L} \times I_{L}+F_{R} \times I_{R} \\
& =\left(\frac{4232}{4256}\right)\left(-\left(\frac{4208}{4232}\right) \log \left(\frac{4208}{4232}\right)-\left(\frac{24}{4232}\right) \times\left(\frac{24}{4232}\right)\right)+ \\
& \left(\frac{24}{4256}\right)\left(-\left(\frac{0}{24}\right) \times \log \left(\frac{0}{24}\right)-\left(\frac{24}{24}\right) \times \log \left(\frac{24}{24}\right)^{1}\right. \\
& =0.994 \times(0.0350)+0.0056 \times(0) \\
& =0.0348
\end{aligned}
$$

The impurity values for tree (b) are 0.0153 (gini) and 0.0299 (deviance). 0.0112 is lower than 0.0153, and that is why tree (a) is the optimal tree based on gini. Similarly, 0.0299 is smaller than 0.0348 , and that is why tree (b) was the optimal tree based on deviance. The computed impurity measures are summarized in Table 2.2.

Table 2.2: Impurity measures calculated using gini and deviance split criteria for the branches in Figure 2.5

\section{Gini criterion Deviance criterion}

\begin{tabular}{lll}
\hline \hline Tree (a) & 0.0112 & 0.0348 \\
\hline Tree (b) & 0.0153 & 0.0299 \\
\hline
\end{tabular}

\subsubsection{Stop criterion}

The tree is built by splitting every node until a pre-defined stop criterion is met. The stopping criterion is the criterion that determines if a node should be divided further into children nodes. Possible stop criteria are minimum samples in parent node and minimum samples in leaf node. To provide a complete picture of the variations in the

\footnotetext{
${ }^{1}$ here, $0 \times \log (0)=0$
} 
data set, the stop criterion can be when all nodes are pure, containing cases of only one class; however this may result in a tree that is over-fitted, meaning is specific to the data set used to create the tree which may increase misclassification for new cases, or branches that are redundant and do not provide much more information than the top branches of the tree when analyzing the tree for classification factors.

\subsubsection{Method for applying the decision tree technique to mushroom data}

Before applying the decision tree technique to infusion data, it is first applied to the mushroom data set to find important factors that differentiate between poisonous and edible mushrooms. Influential factors towards determining mushroom classification will be compared with factors in the known decision tree in Figure 2.2. The decision tree is created with the mushroom data set using a built-in function in MATLAB.

For this analysis, the variable stalk root was eliminated because it had missing values. There are a small number of variables in the mushroom data set; therefore, stopping when all nodes are pure-that is all observations belong to one class- is a feasible option and is ideal for the mushroom data set and viewing the whole tree would provide the most amount of information. Stopping based on any other criteria would not necessarily ensure that all nodes are pure (e.g., using minimum number of cases in a leaf node); though it may take less time and memory. These are considerations to be considered when selecting a stop criterion.

Two trees were created; one using the gini criteria as impurity measure and another using the deviance criteria as impurity measure. To compare the two built trees with the known mushroom rules, the tree in Figure 2.2 is turned into a binary univariate tree in Figure 2.6. To create the new tree with the initial rules, the rules were applied 
to each node in the order that they appear in the rules. The final tree did not have pure leaves, and required the use of an extra variable, the bruises variable, to create two pure leaf nodes. This new binary, univariate tree has seven splits with six variables.

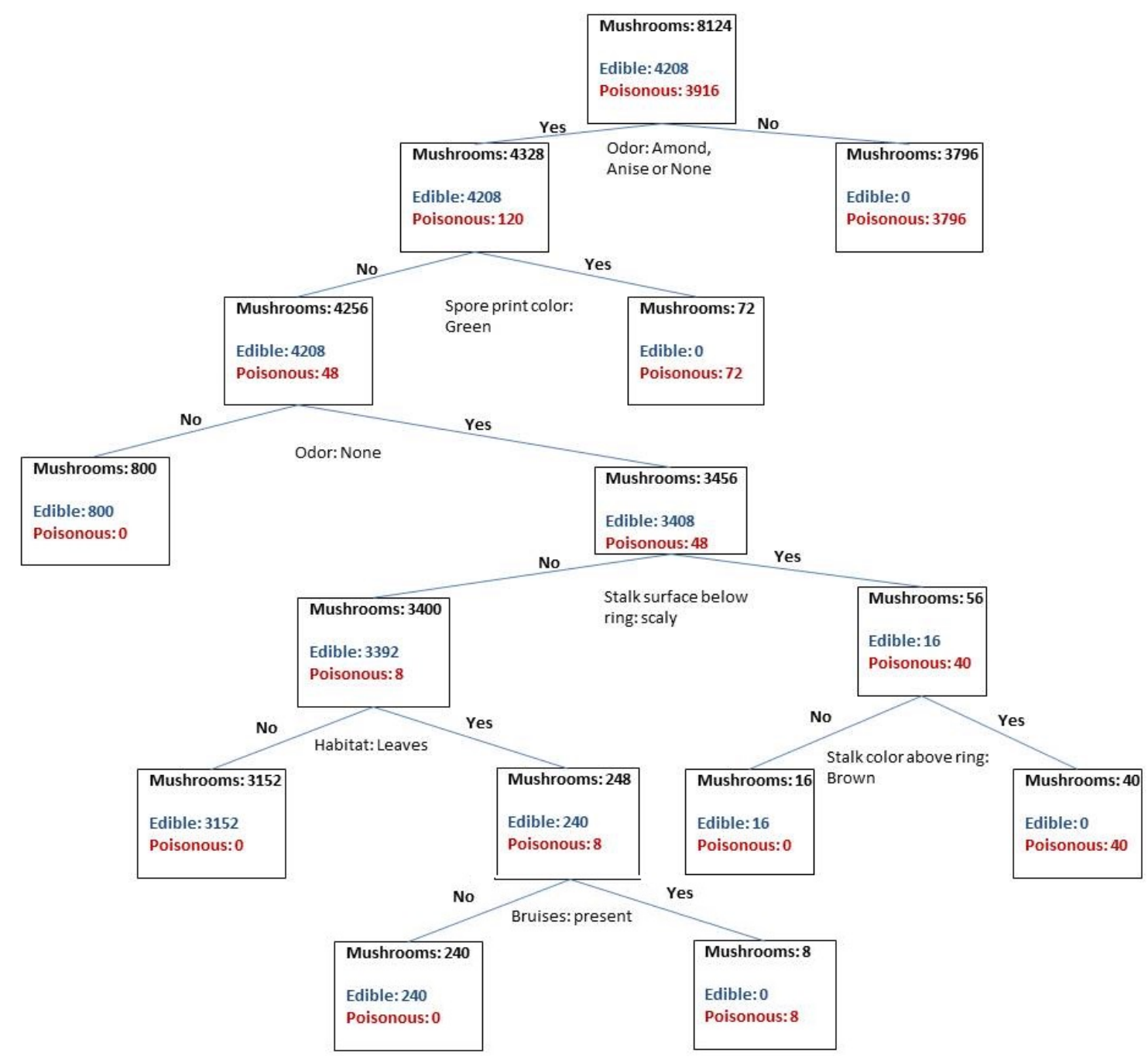

Figure 2.6: Mushroom classification rules converted into a binary univariate decision tree 


\subsubsection{Decision tree results and discussion using mushroom data}

Figures 2.7 and 2.8 show the decision tree built with the gini split criterion and the deviance split criterion respectively. The decision tree technique built using the gini criterion reveals that nine splits that can be represented in nine steps or logical rules and eight attributes are necessary for classifying mushroom data at the end nodes. The decision tree technique built using the deviance criterion classifies the mushroom data with eight splits and seven variables.

In both trees, the first four splits use the same variables odor, spore print color and stalk color below; indicating that they are the four most influential factors. The odor variable is the most influential variable in classifying the mushroom data, consistent with the optimal tree. Both trees have two splits that use the variable stalk color below, which is why each tree uses less variables than splits. The first observed difference in the mushroom data fitted trees is in the third split for the stalk color below variable. The gini criterion splits the cases based on stalk color below ring $=$ brown and not brown; while the deviance criterion splits the cases based on brown or yellow and neither brown nor yellow (everything else). Figure 2.5 in Section 2.2.3 was an example derived from the mushroom trees and highlights this difference between the two trees; it shows the decision tree split for the stalk color below variable; it is split by one value in gini and by two values in deviance. The second observed difference between the two trees is in the number of steps and variables used in each case: gini uses eight variables, while deviance uses seven variables. This may be a consequence of the different third split, which results in a different data set for the fourth split. The third observation is that there are three common variables that are 


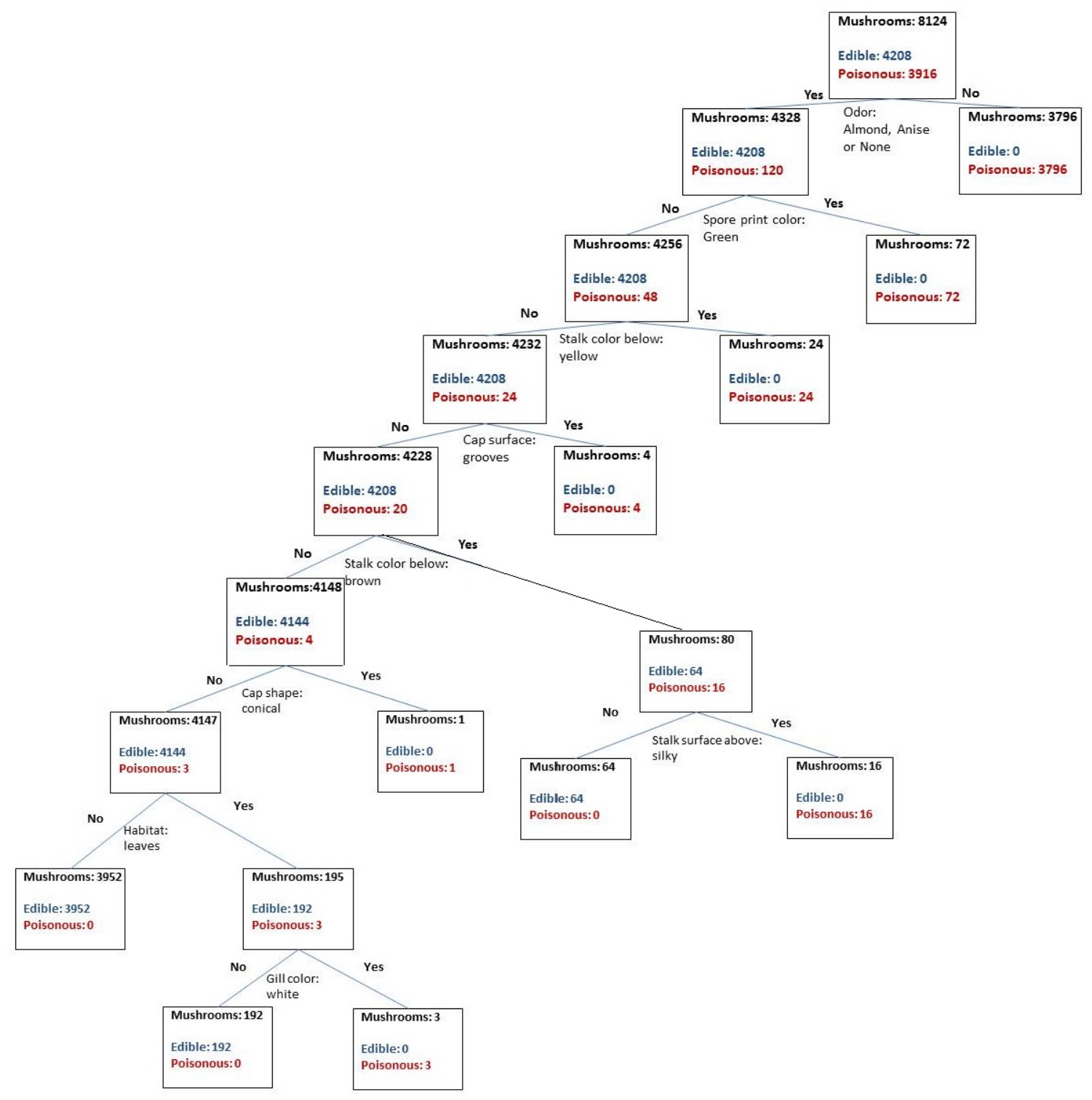

Figure 2.7: Mushroom tree built with the gini criterion 


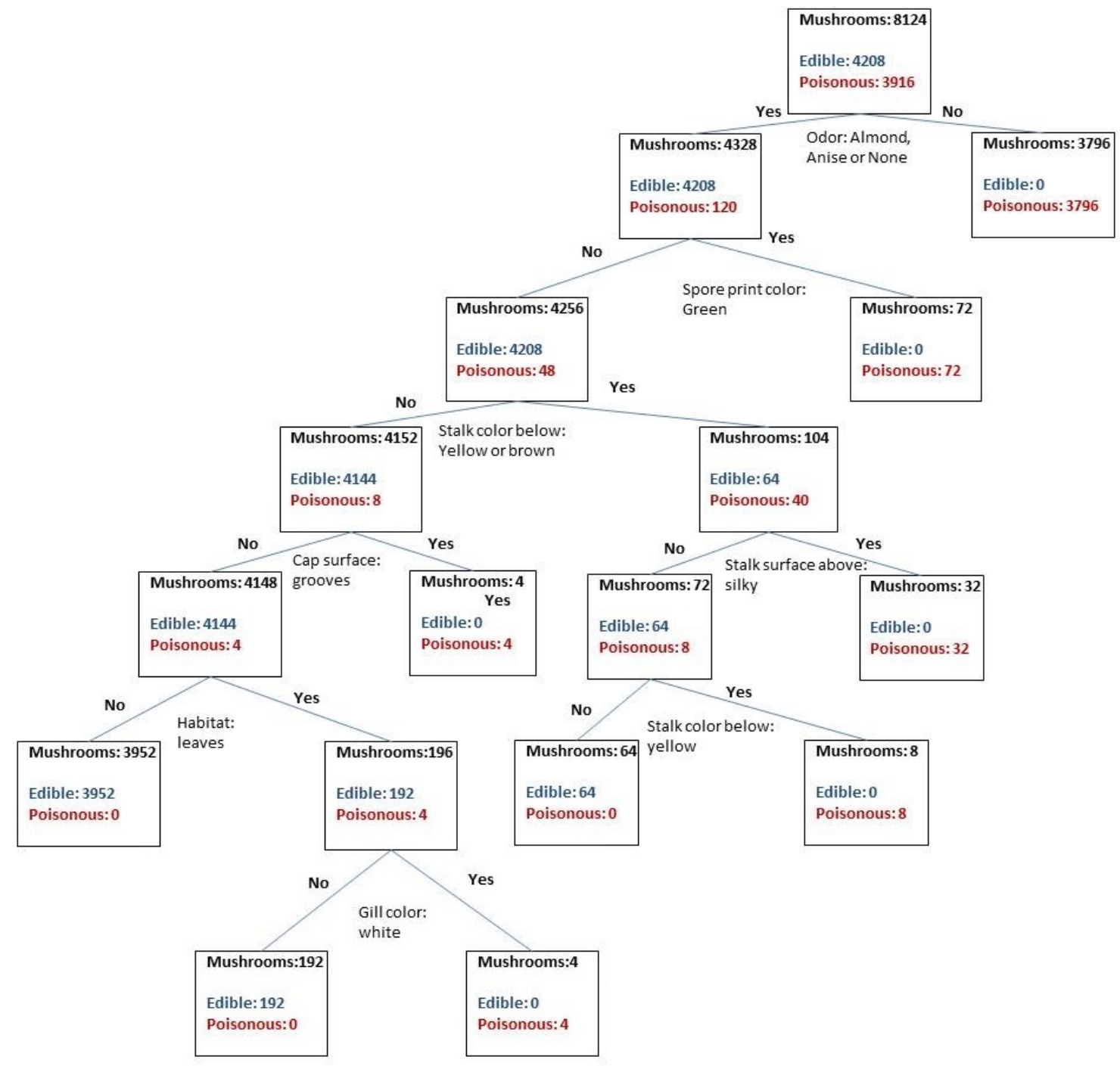

Figure 2.8: Mushroom tree built with the deviance criterion 
also the top three most influential based on optimal rules: odor, spore-print-color and stalk color below ring.

\subsubsection{Conclusions}

The advantage of the decision tree is that it is a white-box method, where the logical steps towards determining the class of a sample from the data set is clearly displayed to the researcher in a visual representation. The decision tree is capable of classifying based on categorical variables in the feature vector; all variables for the mushroom data are categorical and a significant number of variables used for the classification of the infusion data are presented as categorical data as well. The decision tree was appropriate for viewing different variations of classification steps for the mushroom data, in order to highlight the variables influential in classifying the data. For the mushroom data, the deviance criterion gave a smaller tree with one less fewer splits and variables; the optimal binary tree remained the optimal tree, even when the bruises variable was added to create an extra split. In conclusion, based on the information derived from the mushroom decision trees, the characteristics of a decision tree, and similar work by other researchers, the decision tree method is a practical option for using on infusion data. 


\section{Chapter 3}

\section{Analysis of Smart Pump Usage at CHEO}

\subsection{Introduction}

The smart pump research in this thesis was carried out with smart pump data from the Children's Hospital of Eastern Ontario (CHEO) Ottawa, ON, Canada. CHEO is a pediatric health and research center that provides family-centered patient care, breakthrough research and training for future health care practitioners [59].This research is based on CHEO's Medfusion 4000 syringe infusion pump (Smiths Medical, St Paul MN, USA; Figure 3.1) from CHEO. CHEO is the first site in Canada to adopt the Medfusion 4000 pumps and has deployed 185 pumps since December 2010. The Medfusion 4000 is a smart infusion pump with a customizable drug library and device server system that collects therapy data (e.g., drug information entered for each infusion) and alarm data (e.g., empty syringe and hard limit alarms) [60,61]. The data collected are traceable to individual infusion pumps; however, data are not traceable or linked to any patient, patient ID, or clinician. This research was approved by the CHEO Research Ethics Board and Carleton University Research Ethics Board. Data presented in this thesis were extracted from August 2011 to August 2012. 


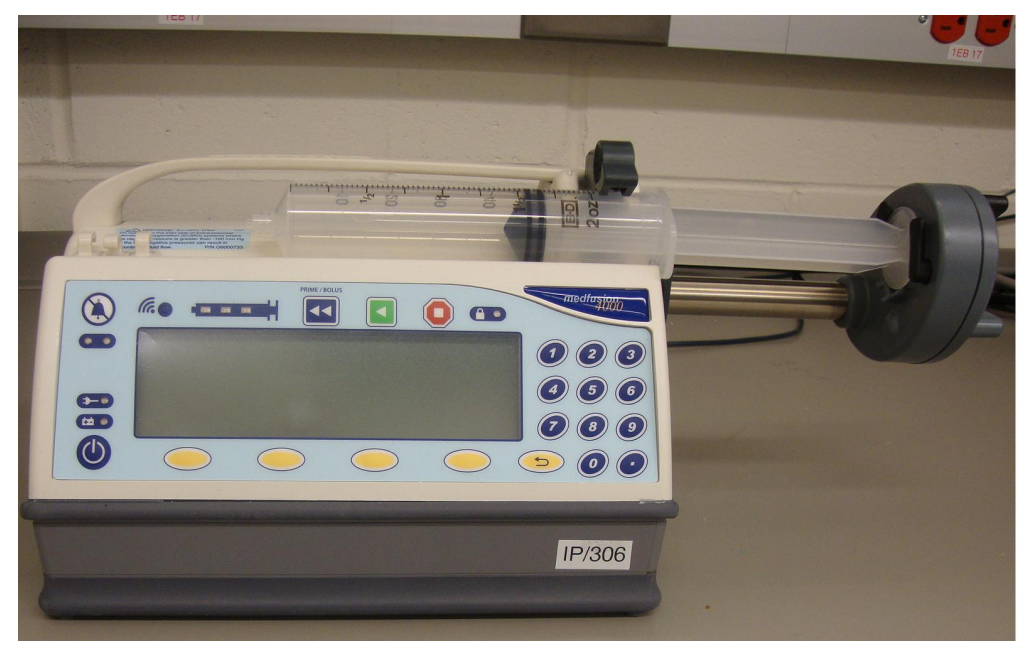

Figure 3.1: Medfusion 4000 Pump

\subsection{The Children's Hospital of Eastern Ontario}

CHEO services the regions of eastern Ontario, parts of northern Ontario, western Quebec, and Nunavut and has an inpatient capacity of 167 beds. CHEO is a pediatric hospital that admits patients as young as pre-mature, newborns and as old as teenagers until their eighteenth birthday. The hospital is also an academic health science center providing education to 2300 future pediatricians, nurses and health professionals, and a research institute that conducts research on issues such as cancer, diabetes, obesity, and genetic disorders that affect the children's population. CHEO has an annual revenue of $\$ 230$ million, conducts $\$ 27$ million of research and employs more than 2500 doctors, nurses and other staff including 250 researchers.

Patients that are treated at CHEO and any children's hospital present challenges unique to the pediatric environment. Patient care at children's hospitals is typically family-oriented. The philosophy is that the well-being of the parents and family is just as important as the patient's well-being because it is the parents who will take care of the patient when they are released. Proper follow-up care is important to ensure proper healing and to avoid hospital revisits [62]. Communicating levels 
of pain can be a challenge between patient and clinicians, especially for children not old enough to speak [63]. Children's hospitals need to acquire equipment for patients of all sizes, e.g., beds, imaging equipment, respiratory masks [63]. Birth rates impact the population of potential patients admitted into the hospital (children have a different disease epidemiology than in the adult population). Children are susceptible to unique risks and more severe forms of certain diseases; for example, birth defects and respiratory illnesses respectively [64].

Prescribing medication to the children's population is also a greater challenge than for the adult population because of varying pharmacokinetic and pharmacodynamics factors in the children's population $[65,66]$. Pharmacokinetics is the study of the passage of drugs through the body. Pharmacodynamics studies the effect of drug dosage on an organ or system of organs. Pharmacokinetics and pharmacodynamics are affected by factors that change with age in a normal developing child: body composition, weight, size and physiology. The large number of factors that influence the effect of drug dosing on the pediatric patient creates more opportunity for errors when prescribing medication, and additionally places a higher risk of harm in the case of an error, because of overall increased sensitivity to dosing amounts. For patient safety purposes, standard dosing is created within health care institutes for clinicians to follow when prescribing medication to patients based on knowledge of those factors. The large portion of pediatric standard dosing is either based on patient weight or based on patient body surface area. Drugs are prescribed based on desired effects and outcomes on the patient's body, and there may be patient cases where the desired outcome requires dosing that exceeds set standards. This creates challenges when using and designing a smart pump drug library that uses standard dosing as starting points for creating the limits in the library.

At CHEO, there is an internal safety reporting system that all personnel have 
access to for reporting medication events or any other safety events that have harmed a patient at CHEO or had the potential to cause harm. Only key personnel have access to viewing all reports for analysis. Director of Pharmacy, Dr. Regis Vaillancourt, receives all medication related incidents causing harm or with potential to cause harm, and Elaine Wong, key actor for patient safety at CHEO, receives all incidents for assessment. Medication incident reports must indicate whether or not there was actual harm or that the event was intercepted, and the level of harm (e.g., no harm, minor harm, major harm, or death). A medication incident committee meets regularly to discuss reported incidents at CHEO.

A patient admitted at $\mathrm{CHEO}$ will have a medical record file which consists of two parts. First, a BPMH (Best Possible Medication History) containing information on all medications and allergies that the patient has, and second, the AMO (Admission Medication Orders) containing information on all medications administered to the patient during their hospital stay.

\subsection{Smart pumps at CHEO}

CHEO has a total of 465 pumps: 185 syringe pumps and 280 large volume pumps. Anesthesia patients undergoing surgery will be receiving medication via at least one infusion pump; and it is not abnormal for patients in the intensive care units to be connected to numerous (at times up to 13) infusion pumps. Syringe pumps are heavily used in children's hospitals because of their ability to deliver small precise amounts of medication. The following sub-sections describe the procedures for drug library updates (Section 3.3.1), verifying patient prescriptions (Section 3.3.2) and the organization of the drug library (Section 3.3.3). 


\subsubsection{Drug library updates}

At CHEO, hospital policy dictates that, at a minimum, drug libraries should be reviewed and updated with pharmacy approved changes at least three times during each year. These library changes take into account new drug additions to the formulary and clinician suggestions. Carmen Ma, a CHEO pharmacist, is the drug library manager and is responsible for maintaining and reviewing the drug library for the Medfusion 4000.

During the study period from August 2011 to August 2012, drug libraries were updated three times on September 22, 2011; March 13, 2012; and on June 21, 2012.

\subsubsection{Prescriptions}

At CHEO, a pharmacist reviews all medication orders for hospitalized patients to verify therapeutic appropriateness. Non-standard prescriptions are discussed between both the medical doctor and the pharmacist. Pharmacists review the patients medical and medication history, allergy status, dosing, dosage form, drug interactions, and side effects of each new medication. Pharmacists also play an active role during multi-disciplinary rounds where the medication plan for each patient is discussed with a team of doctors, nurses and allied health professionals. Pharmacy must also be notified of patients requiring special treatment and other than standard treatments and prescriptions. When a clinician programs an infusion of a prescription outside of the drug library limits, a consultation with a medical doctor is required for programming an infusion outside of the drug library. 


\subsubsection{Profiles and profile categories}

The drug library at CHEO categorizes all drugs into nine separate profiles, largely based on the departments in the hospital (see Table 3.1). There is also a set of profiles used for training and testing that are not used when delivering infusions to actual patients and are not considered in this research. Profiles make it easier for a clinician (nurse or doctor) to find the drug setting when using an infusion pump. Profiles also allow pharmacists to set different limits for the same drug in different profiles; for example, narrower drug dosing range limits are programmed for the neonatal intensive care unit. (e.g., 'ampicillin $100 \mathrm{mg} / \mathrm{mL}$ ' for patients below $40 \mathrm{~kg}$ has a hard limit range of $22 \mathrm{mg} / \mathrm{kg}$ to $105 \mathrm{mg} / \mathrm{kg}$ in the General Peds/Surg profile, and a range of $45 \mathrm{mg} / \mathrm{kg}$ to $110 \mathrm{mg} / \mathrm{kg}$ in the NICU profile. The lower hard limit is larger for the NICU profile because ampicillin is used due to a different indication in that patient unit that would require a larger dose that is given three times a day rather than a smaller dose four times a day.). Profiles are further divided into categories so that the user does not need to scroll down a long list of drugs to find the drug they are delivering. Profile categories divide the drugs in the profile based on alphabetical order. Other categories included for each profile are the Flush and Volume-over-Time categories. The Flush category is designed to empty the infusion lines at a specified rate, and should take a short amount of time to complete. The Volume-over-Time category is designed to delivery an infusion for a drug that is not in the drug library or for a non-standard prescription, and only needs volume and time to be entered. 
Table 3.1: Profiles in the CHEO drug library with description

\begin{tabular}{|c|l||}
\hline Profile & Description \\
\hline \hline Emergency & Profile used in the emergency department \\
\hline PICU & Profile used in the pediatric intensive care unit \\
\hline NICU & Profile used in the neonatal intensive care unit \\
\hline Anesthesia & Profile used for patients undergoing anesthesia \\
\hline Anesthesia CVS & Profile used for patients undergoing anesthesia for \\
Hem/Onc & cardiovascular surgery \\
\hline General Peds/Surg & Profile used for patients in hematology and \\
\hline
\end{tabular}

\subsection{Smiths Medical's Medfusion 4000 syringe in- fusion pump}

The Medfusion 4000 pump is a battery-powered, portable and wireless device. The pump has the option of also being hardwired to the network and/or being plugged-in. The pump's device server is part of Smiths Medical's PharmGuard Server software and has report generating capabilities that provide information to clinicians on the 
usage of the pumps [60]. Drug library updates are sent wirelessly to the pump, and data collected by the pump are sent wirelessly from the pump to the server. Data are accessible through a user interface from the PharmGuard device server. Smart pump data analysis in this thesis is done with data collected from reports generated from the device server. That is, only data presented in PharmGuard reports were analyzed and raw data transferred from the pump to the server were not accessed. Several reports can be generated for presenting collected data in graphical or table formats that are easy to understand. Some of the reports viewed for this thesis are the safety event reports, event history reports, and compliance reports. Safety event reports give information on when soft or hard limits are exceeded for every drug in the library. Event history reports present all pump events (e.g., hard and soft limit events, alarms, library updates, and infusions) in chronological order with details associated with the event (e.g., infusion dose, infusion time, and alarm triggers). The event history report allows a review of pump programming including programming that led to a hard limit. Follow up interventions are also tracked in the event history report. Compliance reports present the number of infusions started in the drug library, and the number of infusions started outside of the drug library.

\subsubsection{Compliance}

An indicator of compliance as computed by the PharmGuard Server software is the ratio between the number of manual mode infusions and the total number of infusions. Manual mode infusions are infusions completed outside of the library and are referred to as non-PharmGuard Infusions. A common non-PharmGuard infusion is a Volumeover-Time infusion, which allows users to enter a volume between 0.1 and $500 \mathrm{~mL}$ and a time between 1 minute and 10 hours for any drug or other solution in the syringe. The ranges are very large and are not specific to the drug or patient's weight, as a 
result, the smart pump is not able to detect order entry mistakes.

Based on individual reports from clinicians, there are other workarounds within the drug library to enter doses or rates that fall outside of the limits. Workarounds are not malicious (i.e., there is no intent of harm) and are reported to be used when limits are too strict and prevent medication delivery to patients that require unique prescriptions. Such workarounds are examples of non-compliance settings that are not detected in the PharmGuard compliance rates, but must also be considered. Known workarounds include:

1. Entering a different patient weight. When limits are weight dependent, falsely increasing the entered patient weight could increase the limits.

2. Entering the drug from a different drug library list. In the PharmGuard system, the different drug lists are referred to as profiles and are used to categorize drugs. Different profiles may have the same drug with different soft and hard limit ranges, and

3. Entering a different drug program in the list. In the CHEO drug library, one drug may have different soft and hard limits based on several factors, such as the number times it is administered in the day. Therefore, it will be listed as two separate drug programs with an indication of the difference at the end of the name (e.g., gentamicin traditional, which is administered three times a day, and gentamicin once daily).

Increase in non-compliance resulting from such workarounds is a concern since the pump depends on the user to use it in the way it was intended so that the drug library can help reduce drug errors. The device server may contain false data if an improper weight, drug or profile is entered. Turning off the safety features or entering false data 
to the pump to get around library limitations are issues that should be addressed in training along with the potential risks.

\subsubsection{Drug library updates}

When a new library is ready to go, the deployment of the old version is stopped on the server and the deployment of the new version is started. There are three states for the library update that the server logs by date and time for each pump. The first state is the notification by the server to the pump that there is an update or new library. The second state is the transfer of the library file to the pump, and the third state is the validation back to the server confirming that the new library is active and in use on the pump.

When a pump is first powered on and there is a wireless connection, it takes approximately ten minutes for the notification and transfer states to occur for pumps that require the update. At this point, the pump is still used with the old library and is used for however long it is required by the user. The actual library update on the pump only takes place at the next power off. When a power off is initiated, the user is prompted "update available. Select yes to enable update or no to power off"; if the user is in a rush and still needs the pump, they can bypass the update and power off and on and continue to use the old library. If the user selects yes to the library update, the pump updates and turns off; at this point, the third state is still not confirmed. The final state is only confirmed at the next power on (within ten minutes on with wireless).

A deployment is always enabled and active for the server to catch outlier pumps that might not have been powered on or had wireless activity. It typically takes one week for approximately $95 \%$ of pumps to be updated with the new library at CHEO. This delay between a new library update and an update being used on the pump 
means that there are discrepancies when analyzing data before and after the date of a new library deployment, because not all pumps will necessarily be using the new library directly after.

\subsubsection{Programming an infusion}

In general, programming a smart infusion pump takes more time than a traditional pump when using a drug library. To setup any infusion (PharmGuard or nonPharmGuard), the profile is selected first. Setting up a smart pump includes the selection of the drug and entering the patient's weight or body surface area twice in the cases where the drug needs a weight-based dosing. Not only does setting up an infusion pump take more time because of the higher number of steps required, but it also can also introduce additional errors at every step where a selection needs to be made (e.g., profile or drug selection) and/or where a value needs to be entered (e.g., weight, dose, time or rate). The steps below give a more detailed explanation of the programming steps and the example in Figures 3.2 and 3.3 show the pump programming steps for "gentamicin $5 \mathrm{mg} / \mathrm{mL}$ once daily" in the General Peds/Surg Profile using the Medfusion 4000.

\section{Step 1: Turn pump ON}

Step 2: Place syringe in holder The pump is designed to hold a syringe size of 1, 3, 5,10, 20, 30 and $60 \mathrm{~mL}$ that clips onto the pump in a specific manner. (Figure 3.2 a)

Step 3: Select profile and profile category The profile is selected, then the profile category is selected. The profile is further divided into alphabetical categories for finding drugs easier and quicker. (Figure $3.2 \mathrm{~b}$ and $\mathrm{c}$ ) 
Step 4: Select drug (Figure 3.2 d)

Step 5: Confirm drug selection The purpose of confirming the drug is to ensure that the wrong drug was not selected accidentally. (Figure $3.2 \mathrm{e}$ )

Step 5: Enter weight (twice) Certain drugs have dosing limits based on patient weight or body surface area. These variables are entered before the dose is entered if that is the case. The weight parameter has hard limits from 0 to 250 $\mathrm{kg}$. Because of the large variation in patient weight, the weight needs to be entered twice to confirm correct entry. (Figure $3.2 \mathrm{f}$ and $\mathrm{g}$ )

Step 6: Enter dose Dose prescribed is entered. (Figure $3.2 \mathrm{~h}$ )

Step 7: The infusion time is entered Length of infusion is entered. There are also hard limits for time for certain drugs. (Figure $3.3 \mathrm{i}$ )

Step 8: Confirm infusion parameters Infusion parameters are displayed on screen for the clinician to double check and confirm. (Figure $3.2 \mathrm{j}$ and $\mathrm{k}$ )

Step 9: Start infusion Once again, infusion parameters are displayed and the pump prompts the user to enter begin. The screen displays infusion parameters and time left in minutes during the infusion. (Figure 3.2 l) 


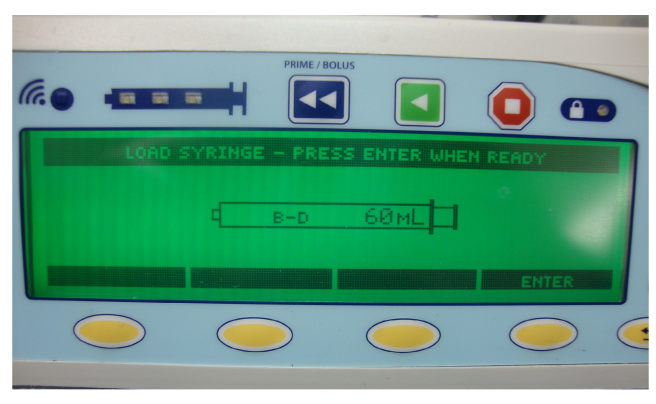

a.

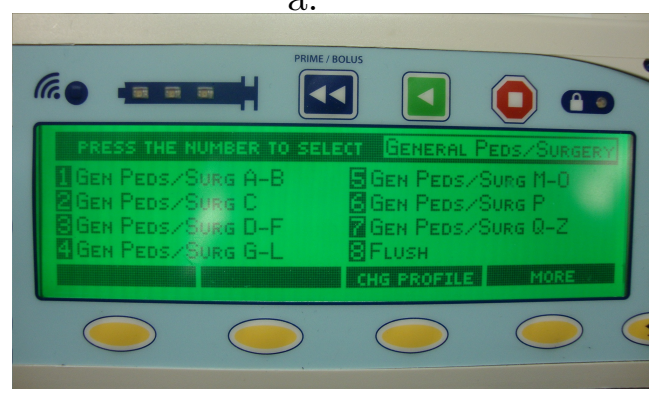

c.

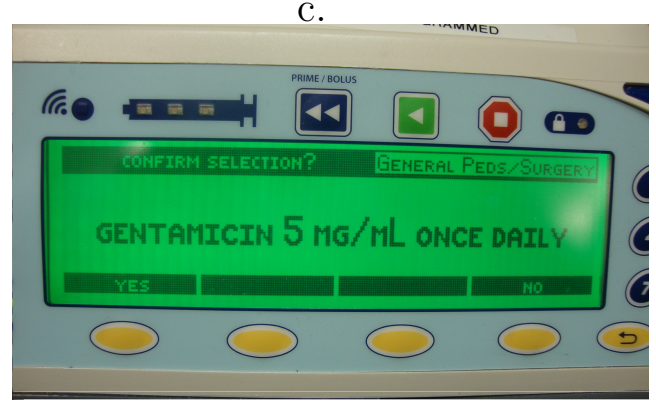

e.

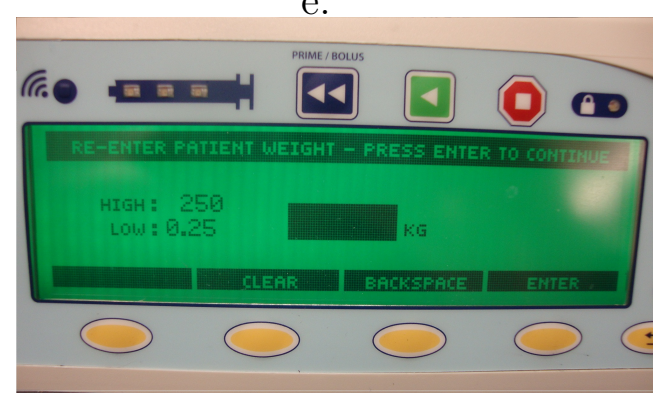

g.

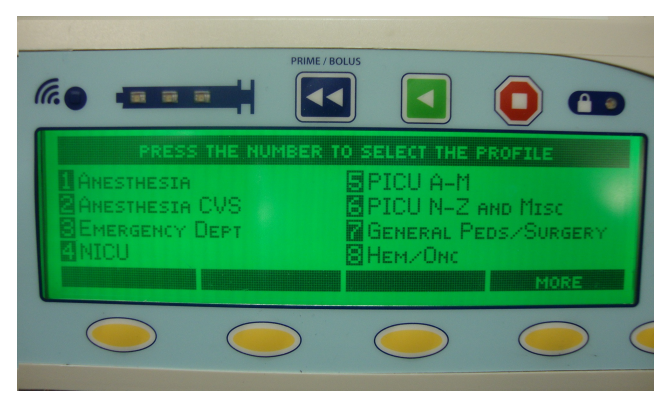

b.

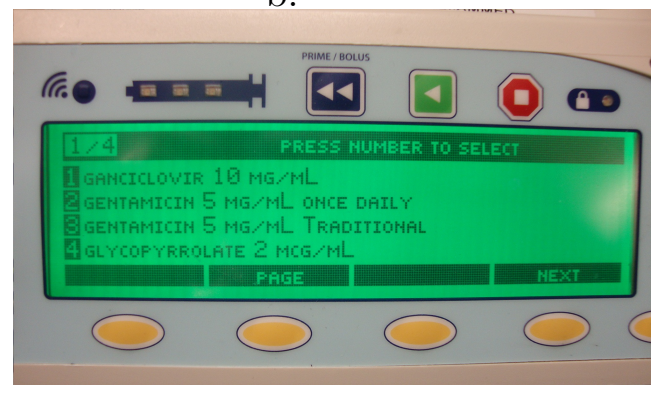

d.

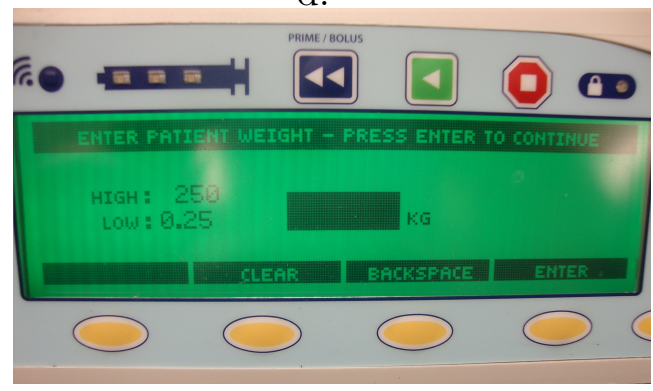

f.

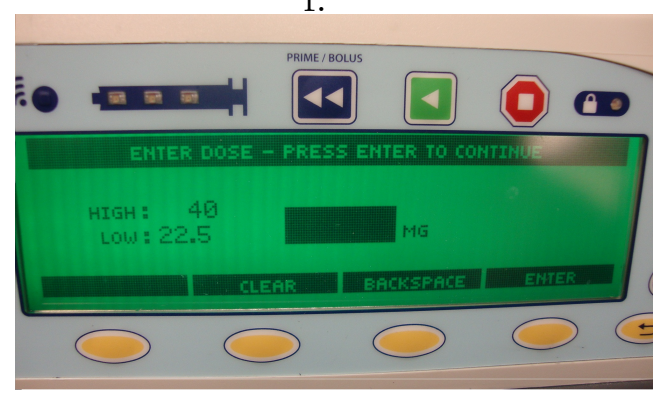

h.

Figure 3.2: Pump programming steps 2-6: Images taken of the Medfusion 4000 for programming a "gentamicin $5 \mathrm{mg} / \mathrm{mL}$ once daily" infusion in the General Peds/Surg profile 


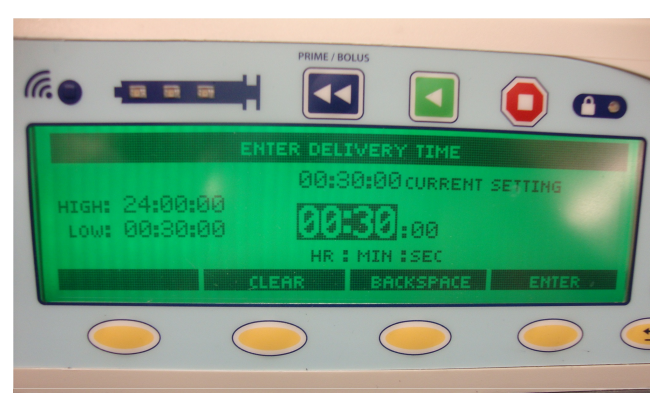

i.

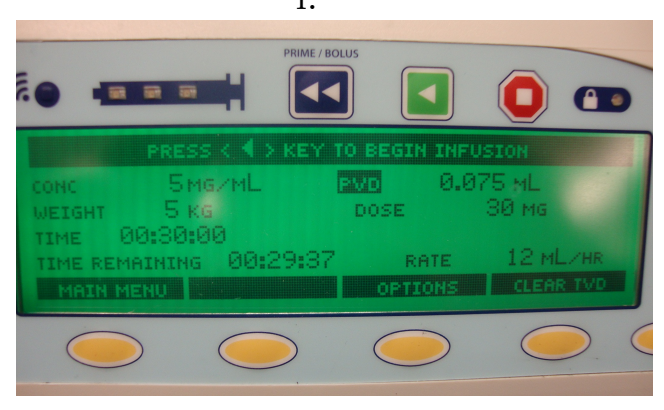

$\mathrm{k}$.
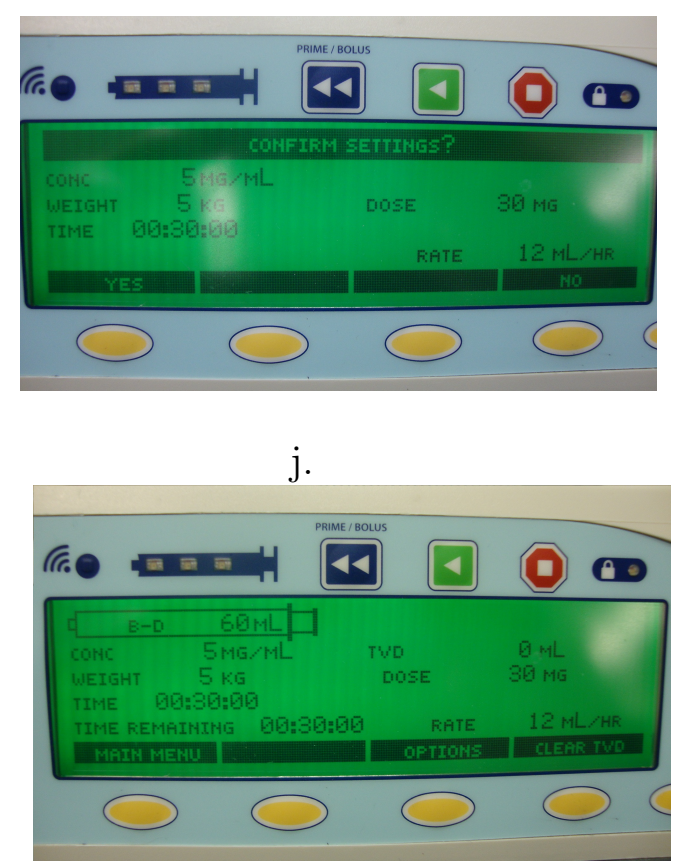

1.

Figure 3.3: Pump programming steps 7-9: Images taken of the Medfusion 4000 for programming a "gentamicin $5 \mathrm{mg} / \mathrm{mL}$ once daily" infusion in the General Peds/Surg profile

For programming a Volume-over-Time infusion, a type of non-PharmGuard infusion option in the General Peds/Surg profile, steps 1 to 3 are identical except that the Volume-over-Time category is selected instead of a profile category that would direct the user to a drug list. From there, the steps are different and are shown below:

Step 4: Enter infusion volume This value also has programmable limits, but the range is very wide, making it difficult to detect programming errors.

Step 5: Enter infusion time Just like volume, the value has programmable limits with a very wide range (minutes to hours).

Step 6: Confirm infusion parameters Infusion parameters are displayed on screen for the clinician to double check and confirm. (Figure $3.4 \mathrm{j}$ ) 
Step 7: Start infusion Once again, infusion parameters are displayed and the pump prompts the user to enter begin. The screen displays infusion parameters and time left in minutes during the infusion.

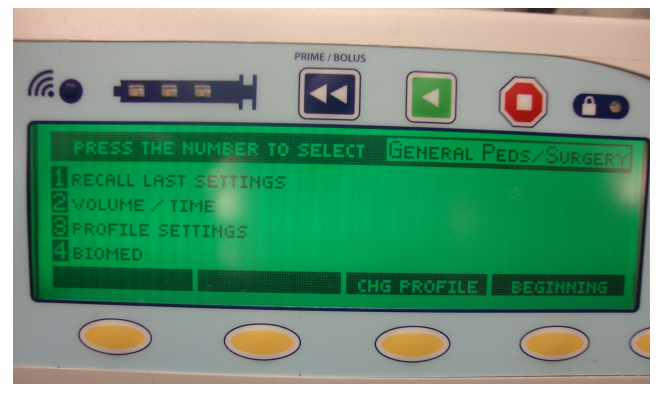

a.

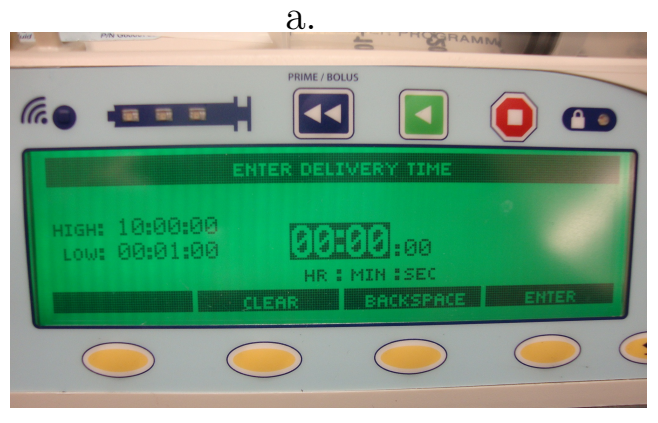

c.

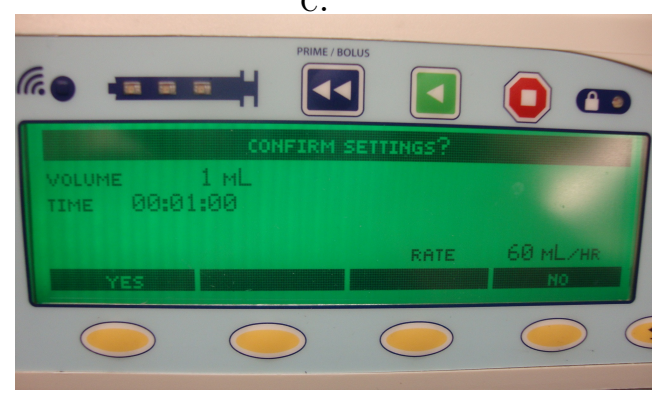

e.

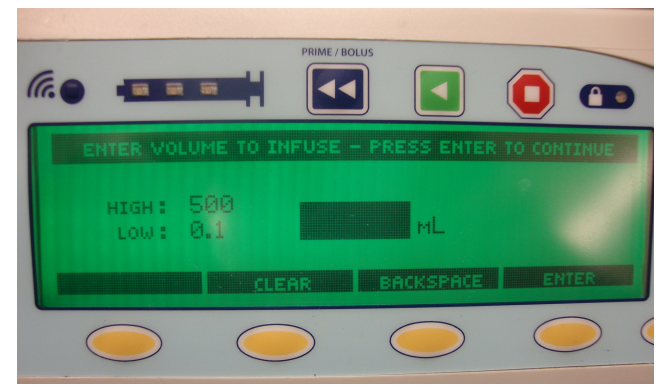

b.

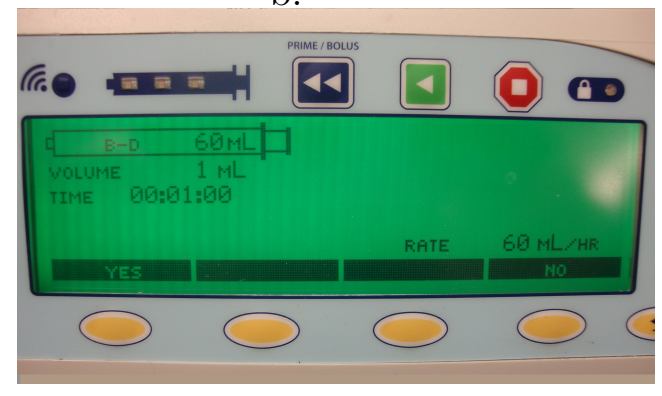

d.

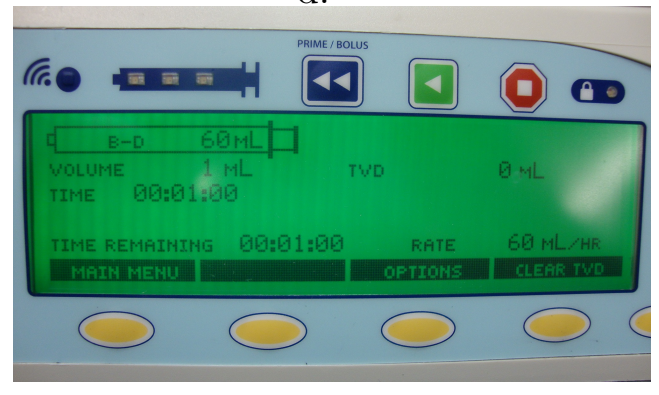

f.

Figure 3.4: Pump programming steps 4-7: Images taken of the Medfusion 4000 for programming a Volume-over-Time infusion

It takes a minimum of 9 steps to program a gentamicin infusion and a minimum of 7 steps to program a Volume-over-Time infusion. If a soft or hard limit was hit, then it would take an extra step for re-entering the value or overriding the warning 
(in the case of a soft limit override). When a hard limit is hit (Figure 3.5), the pump does not go to the next setup step screen until a value is entered within the limits displayed. In Figure 3.5, the soft limits are represented as "low" and "high" values, and the hard limits are represented as "min" and "max" values. In this figure, the soft and hard limits are the same: from 30 minutes to 24 hours. When soft limits are equal to the hard limits, that means that there are basically no soft limits. The value of the default delivery time for gentamicin is 30 minutes and is displayed at the center top, over the entry field. In this case, a delivery time of 15 minutes was attempted, but was below the lower hard limit of 30 minutes. Other than the soft and hard limits being displayed on the left of the screen, there is no other visual cue or message that the limit was exceeded- it is up to the user to double check the entered value with the limits. When a soft limit is hit (Figure 3.6), an alarm is sounded and the pump displays the soft limits and hard limits as well, and asks the user if they want to confirm the limit override. To enter a different value, the user must answer "no" and enter the new value on a different screen. To keep the entered value, the user must answer "yes". 


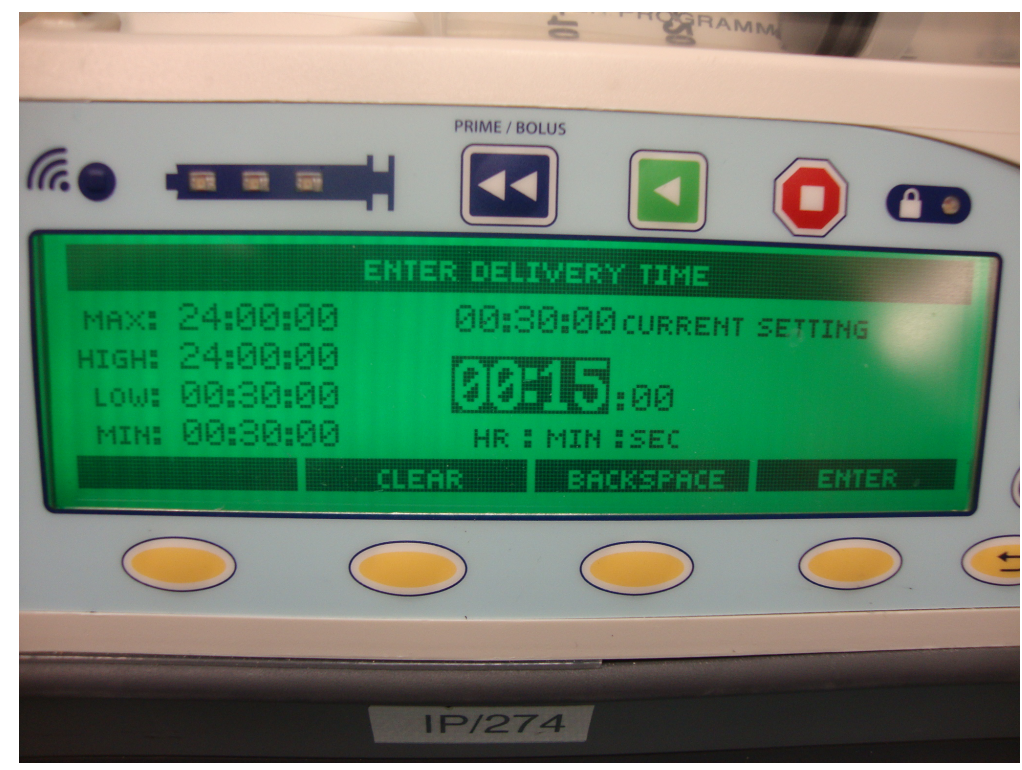

Figure 3.5: Example of hard limit event when entering infusion time. A hard limit event is the event where a clinician enters a value outside the Hard limit events in the drug library.

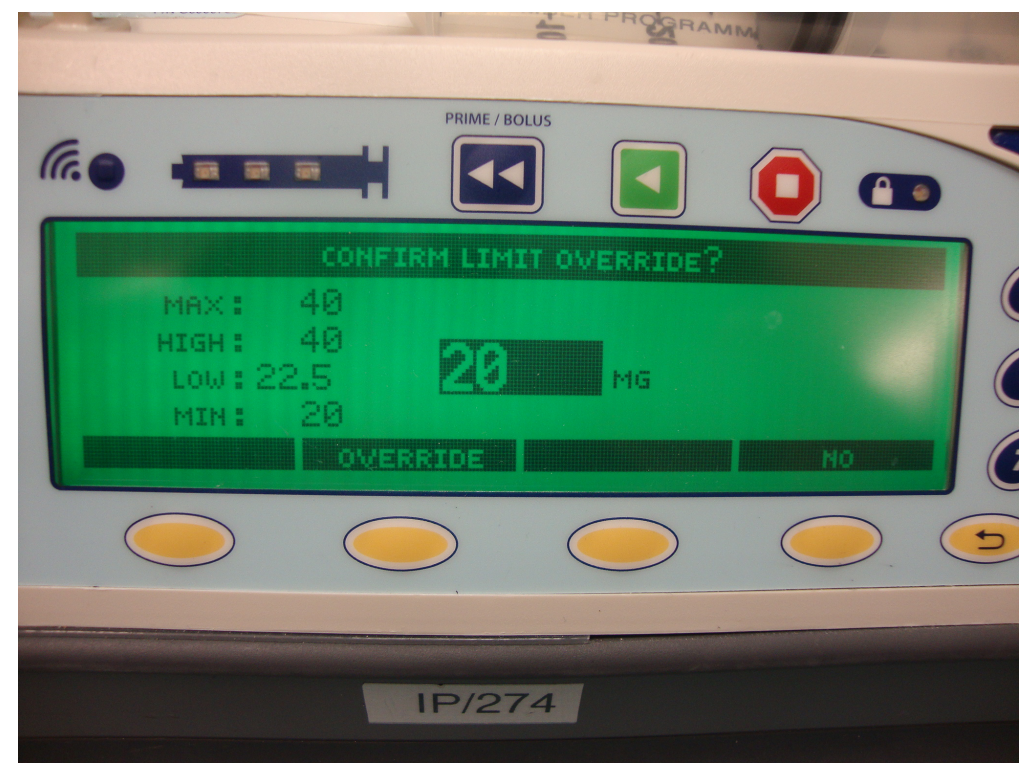

Figure 3.6: Example of Soft limit event override event when entering dose. A soft limit event is the event where a clinician enters a value that is outside of the soft limit but inside of the hard limits in the drug library. 


\subsubsection{The Event History report}

The event history report is used to collect data for this thesis. The events most used in this thesis are recorded are described in this section along with relevant variables of interest in this thesis. Examples of the events can be found in Appendix A. Event reports have six columns:

1. Serial number: The serial number of the pump

2. Date and Time: The date and time of the event

3. Event type: A description of the type of event (e.g., power)

4. Trigger: A description of the event type (e.g., for an event type "power", trigger will be equal to "on" or "off")

5. Message: A message giving more insight on the event. (e.g., "Normal power up complete")

6. Details: A list of variables that give information on the infusion attempted to being delivered when the event occurred. (e.g., power source is a variable that can be equal to "AC" or "Battery")

\section{Therapy-Setup event}

The Setup event is saved after profile and category are selected, but before infusion values such as weight, dose and time are entered. The Setup event will have a "profile' variable, an 'infusion type' variable and a 'category' variable. A PharmGuard infusion will also have a 'drug' variable, which is the main variable identifying if the infusion is PharmGuard or non-PharmGuard. Normally, if the values of the infusion parameters are entered within the drug library limits and no other issues or alarms are encountered, the event coming after the Setup event is the Begin event. 


\section{Therapy-Begin event}

The Begin event is saved at the start of an infusion. Variables saved in this event are relevant to the type of infusion programmed. For instance, a non-PharmGuard infusion will not have a 'drug' variable. A bolus infusion will have a 'bolus dose' variable, a loading infusion will have a 'loading dose', and a continuous infusion will only have a 'dose' variable. Also, the 'weight' and 'body surface area' variables may or may not appear depending on the drug selected. The Begin event does not have a 'profile' or 'category' variable.

\section{Therapy change-Hard limit event}

The Hard limit event as referred to in this thesis is saved as a therapy change event with "Hardlimit" as the trigger variable after the first step taken after a hard limit value is exceeded. The Hard limit event saves the 'profile' variable, the 'drug' variable, the 'upper' and 'lower' hard limits, the parameter that was entered (e.g., weight, dose, time or other), the 'original value' variable, the 'entered value' variable, and the 'final value' variable. The 'original value' is the default value for that parameter; a default value is not always pre-set and can be equal to zero. The 'entered value' is the value which triggered the hard limit event. The 'final value' is the new value that was entered, and is normally a value within the hard limit range. If the user entered the 'backspace' button, either to restart the infusion setup or to return to re-enter the value in the previous screen, a value of zero is saved in the 'final value' variable.

\section{Therapy-Warning and Therapy-Override events}

A Therapy Change event with "Warning" as the trigger variable is when a soft limit is exceeded and a different value is entered when a warning is presented to the user on screen. A Therapy Change event with "Override" as the trigger variable is when 
a soft limit is exceeded and the warning is overridden so that the original value is set and unchanged. The parameters in these events are identical to those for the Hard limit event, but the hard limit 'upper' and 'lower' variables are replaced by the soft limit 'upper' and 'lower' variables.

\subsection{Stakeholders}

The deployment, implementation, and maintenance of smart infusion pumps involves several stakeholders. The stakeholders are the pump users, pharmacists, clinical engineers, biomedical engineering technologists, information technologists, pump manufacturers, and patient.

Pump user: A clinician, who is a nurse or doctor, that sets up the pump and program it for an infusion.

Educator: Nurses and clinical engineers who are responsible for training pump users on operation and educating them on its benefit.

Drug library manager: The drug library manager, usually a pharmacist, creates and updates the drug libraries. He/she also monitors the usage of drug library by reviewing compliance, limit events and other infusion data.

Clinical engineer: A clinical engineer is involved in researching and purchasing of smart infusion pumps based on needs expressed by pump users and patient safety standards. Also keeps up to date with smart pump research and usage in the hospital.

Biomedical engineering technologist: A biomedical technologist maintains and repairs smart pumps. 
Information technical analyst: An information technical analyst maintains wireless infrastructure, and ensures that pump and server software are secure and updated on a continuous basis.

Smart pump company: A company that develops, manufactures or sells smart pumps.

Patient: A patient from CHEO between the age of 0 to 18 years that is administered medication or nutrition via the smart pump.

Unlike traditional pumps, smart pump systems require collaboration and active participation of several stakeholders. Smart pump systems are a recent technology that are being implemented in health care institutions; therefore, collaboration and communication between manufacturers and clinical engineers are necessary for successful implementation and continuous improvement of the system. Manufacturers must provide thorough training to key educators on how to use the pump, to the drug library manager on how to create and update the drug library, and to clinicians using the server database for viewing pump data. This is followed by training and education by educators to clinicians on the importance of compliance in improving patient safety. Presenting cases and reports that show when the drug library prevented an error at nurse meetings or in weekly/monthly newsletters are useful education tools for motivating the use of smart pumps and increasing compliance. Creation of the drug library requires collaboration between clinicians and pharmacists on establishing standard drug dosing limits for all drugs delivered with the infusion pump. Procedures should be established between clinicians and pharmacists for prescribing a drug outside of the standard dosing limits to avoid and/or bypass hard limit alarms. In the case of any issues with interpreting data and reports from pump servers, a contact with the manufacturer should be available to help in understanding the data. Drug 
library changes and updates should be announced to raise awareness on the changes made to avoid confusion when the pump works differently than usual.

\subsection{Examining compliance and hard limit trends at CHEO}

The purpose of this preliminary work is to analyze compliance rate changes over time and Hard limit event rate changes which lead to inefficient use of the infusion pump. The Medfusion database allows such retrospective analysis through the extraction of PharmGuard report data. This initial work is the first step to better understand the usage of the drug library.

\subsubsection{Methodology}

Event History reports were generated from the PharmGuard (PG) database server from August 2011 to August 2012 (thirteen months of data). First, MSExcel was used to extract the Hard limit (HL) events, which are recorded when a hard limit is reached from PharmGuard Event History reports. Second, MATLAB was used to identify Hard limit events that were directly followed by a non-PharmGuard (nonPG) infusion from the reports. Compliance rates, HL event rates, and rates for HL events followed by a non-PG infusion over all infusions and over all HL events were calculated for every month of the study period.

Non-compliance rates over the study period and three months beyond the study period (August 2011 to November 2012) for the Hem/Onc profile were analyzed due to the observed high rates of non-compliance. Non-compliance rates over the study period were also plotted for the Anesthesia profile due to the observed high rates 
of non-compliance. Specifically, a comparison of the rate following a compliance reminder in July 2012 given to nurses at a nurse meeting in the hematology and oncology department is observed to assess effect of training on the compliance. Abbreviations and formulas used in the analysis of this section are listed below.

$I_{T}=$ Total number of infusions

$I_{P G}=$ Total number of PharmGuard infusions

$I_{n P G}=$ Total number of non-PharmGuard infusions

$H L_{T}=$ Total number of hard limit events

$H L_{n P G}=$ Total number of hard limit events followed by a non-PG infusion

Compliance rate $=\frac{I_{P G}}{I_{T}} \times 100$

Non-compliance rate $=\frac{I_{n P G}}{I_{T}} \times 100=1-\frac{I_{P G}}{I_{T}} \times 100$

Hard limit event rate $=\frac{H L_{T}}{I_{T}} \times 100$ 
Rate of infusions where a HL event is followed by a non-PG infusion $=$

$\frac{H L_{n P G}}{I_{T}} \times 100$

Rate of HL events that are HL followed by a non-PG infusion = $\frac{H L_{n P G}}{H L_{T}} \times 100$

Rate of non-PG infusions that are non-PG infusion preceded by a HL event=

$\frac{H L_{n P G}}{I_{n P G}} \times 100$

\subsubsection{Results and discussion}

Tables 3.2 and 3.4 summarize the gathered statistics by month and profile, respectively. The analysis revealed that there were a total of $H L_{T}=3624 \mathrm{HL}$ events for dose, time, weight, body surface area or rate.; 3316 of which were for PG infusions (within the drug library profiles in Table 3.1), and 308 from non-PG infusions. Recall that the Volume-over-Time option, a non-PG infusion option, had limits for volume and time. $H L_{n P G}=213$ of the HL events (about $6 \%$ ) were followed by a non-PG infusion begin event. Compliance reports showed that for the 13 month study period, CHEO's compliance rates were around $\frac{I_{P G}}{I_{T}}=85 \%$ overall. Graphs are presented and 
Table 3.2: Hard limit event and infusion statistics by month: total infusions, $I_{T}$; PharmGuard infusions, $I_{P G}$; non-PharmGuard infusions, $I_{n P G}$; total hard limit events, $H L_{T}$; total hard limit events followed by a non-PharmGuard infusion, $H L_{n P G}$

\begin{tabular}{|l|l|l|l|l|c|}
\hline Month & $I_{T}$ & $I_{P G}$ & $I_{n P G}$ & $H L_{T}$ & $H L_{n P G}$ \\
\hline \hline August 2011 & 4105 & 3967 & 138 & 239 & 6 \\
September 2011 & 4965 & 4767 & 198 & 287 & 2 \\
October 2011 & 6447 & 6159 & 288 & 336 & 9 \\
November 2011 & 5874 & 5526 & 348 & 282 & 10 \\
December 2011 & 6125 & 5330 & 795 & 249 & 11 \\
January 2012 & 6694 & 6136 & 558 & 292 & 18 \\
February 2012 & 6474 & 5889 & 585 & 284 & 24 \\
March 2012 & 6103 & 5413 & 690 & 277 & 20 \\
April 2012 & 6102 & 5184 & 918 & 287 & 28 \\
May 2012 & 6898 & 5634 & 1264 & 263 & 29 \\
June 2012 & 6353 & 5552 & 801 & 300 & 24 \\
July 2012 & 4932 & 4391 & 541 & 220 & 12 \\
August 2012 & 5197 & 4623 & 574 & 308 & 20 \\
\hline Total & 76269 & 68571 & 7698 & 3624 & 213 \\
\hline
\end{tabular}

discussed in the following order: Plot 1 (a graph of $\frac{H L_{T}}{I_{T}}$ and $\frac{H L_{n P G}}{H L_{T}}$ by month), plot 2 (a graph of $\frac{I_{n P G}}{I_{T}}$ and $\frac{H L_{T}}{I_{T}}$ by month), plot 3 a graph of $\frac{I_{n P G}}{I_{T}}$ by profile, plot 4 , a graph of $\frac{I_{n P G}}{I_{T}}$ for the Hem/Onc profile by month over a 16-month period, and plot 5 , a graph of $\frac{I_{n P G}}{I_{T}}$ for the Anesthesia profile. 
Table 3.4: Hard limit event and infusion statistics by profile: total infusions, $I_{T}$; PharmGuard infusions, $I_{P G}$; non-PharmGuard infusions, $I_{n P G}$; total hard limit events, $H L_{T}$; total hard limit events followed by a non-PharmGuard infusion, $H L_{n P G}$

\begin{tabular}{|l|l|l|l|l|c|}
\hline Profile & $I_{T}$ & $I_{P G}$ & $I_{n P G}$ & $H L_{T}$ & $H L_{n P G}$ \\
\hline \hline Hem/Onc & 15794 & 11085 & 4709 & 446 & 49 \\
Anesthesia (Ane) & 2024 & 1942 & 82 & 99 & 0 \\
Anesthesia CVS (AneCVS) & 199 & 137 & 62 & 24 & 0 \\
NICU & 6794 & 5774 & 1020 & 459 & 45 \\
PICU & 10997 & 10488 & 509 & 608 & 13 \\
MDU & 81 & 75 & 6 & 24 & 0 \\
Emergency (Emerg) & 2773 & 2510 & 263 & 254 & 13 \\
General Peds/Surg (Gen) & 37607 & 36560 & 1047 & 1402 & 93 \\
\hline Total & 76269 & 68571 & 7698 & 3316 & 213 \\
\hline
\end{tabular}

Table 3.5: Infusion statistics by month for the Hem/Onc profile from August 2011 to November 2012: total infusions, $I_{T}$; PharmGuard infusions, $I_{P G}$; nonPharmGuard infusions, $I_{n P G}$

\begin{tabular}{|l|l|l|l|c|}
\hline Month & $I_{T}$ & $I_{P G}$ & $I_{n P G}$ & (\%) $\frac{I_{n P G}}{I_{T}}$ \\
\hline \hline August 2011 & 403 & 382 & 21 & 5.2 \\
September 2011 & 413 & 390 & 23 & 5.6 \\
October 2011 & 1090 & 1014 & 76 & 7.0 \\
November 2011 & 934 & 788 & 146 & 15.6 \\
December 2011 & 1733 & 1140 & 593 & 34.2 \\
\hline \multicolumn{5}{|c|}{ Continued on next page } \\
\hline
\end{tabular}


Table 3.5 - continued from previous page

\begin{tabular}{|l|l|l|l|c|}
\hline Month & $I_{T}$ & $I_{P G}$ & $I_{n P G}$ & (\%) $\frac{I_{n P G}}{I_{T}}$ \\
\hline \hline January 2012 & 1395 & 1096 & 299 & 21.4 \\
February 2012 & 1268 & 896 & 372 & 29.3 \\
March 2012 & 1502 & 1031 & 471 & 31.4 \\
April 2012 & 1462 & 900 & 562 & 38.4 \\
May 2012 & 2156 & 1203 & 953 & 44.2 \\
June 2012 & 1419 & 880 & 539 & 38.0 \\
July 2012 & 974 & 610 & 364 & 37.4 \\
August 2012 & 1045 & 755 & 290 & 27.8 \\
September 2012 & 899 & 636 & 263 & 29.3 \\
\hline October 2012 & 909 & 759 & 150 & 16.5 \\
\hline Total & 1084 & 904 & 180 & \\
\hline
\end{tabular}


Table 3.6: Infusion statistics by month for the Anesthesia CVS profile from August 2011 to August 2012: total infusions, $I_{T}$; PharmGuard infusions, $I_{P G}$; nonPharmGuard infusions, $I_{n P G}$

\begin{tabular}{|c|c|c|c|c|}
\hline Month & $I_{T}$ & $I_{P G}$ & $I_{n P G}$ & $(\%) \frac{I_{n P G}}{I_{T}}$ \\
\hline August 2011 & 16 & 12 & 4 & 25 \\
\hline September 2011 & 24 & 14 & 10 & 42 \\
\hline October 2011 & 18 & 12 & 6 & 33 \\
\hline November 2011 & 17 & 10 & 7 & 41 \\
\hline December 2011 & 8 & 6 & 2 & 25 \\
\hline January 2012 & 24 & 10 & 14 & 58 \\
\hline February 2012 & 9 & 4 & 5 & 56 \\
\hline March 2012 & 12 & 12 & 0 & 0 \\
\hline April 2012 & 16 & 12 & 4 & 25 \\
\hline May 2012 & 18 & 14 & 4 & 22 \\
\hline June 2012 & 15 & 13 & 2 & 13 \\
\hline July 2012 & 8 & 5 & 3 & 38 \\
\hline August 2012 & 14 & 13 & 1 & 7 \\
\hline Total & 199 & 137 & 62 & 31 \\
\hline
\end{tabular}


$\frac{H L_{T}}{I_{T}}$ and $\frac{H L_{n P G}}{H L_{T}}$ rate plots by month:

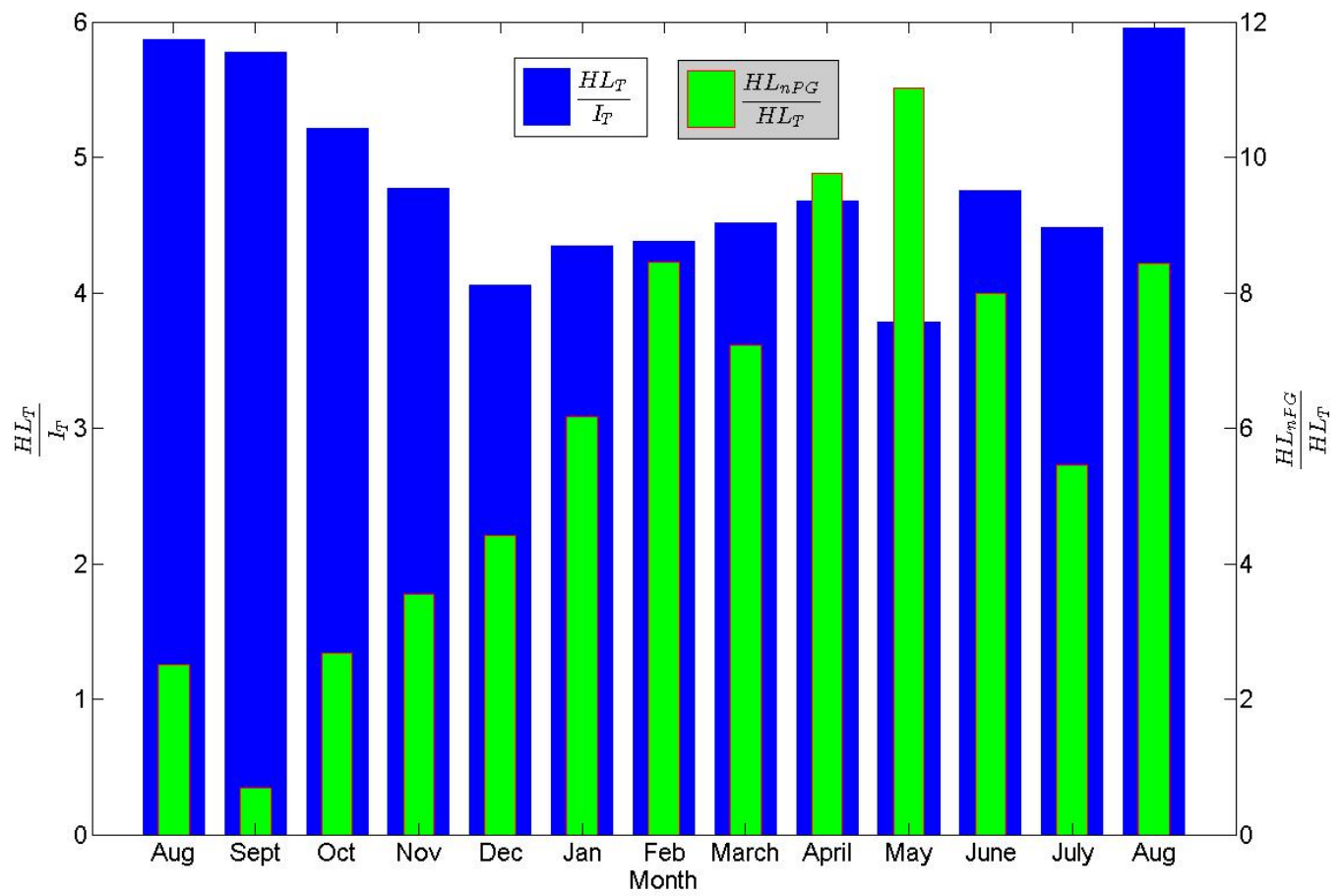

Figure 3.7: The percent of hard limit events over all infusions, $\left(\frac{H L_{T}}{I_{T}}\right)$, and the percent of hard limit events followed by a non-PharmGuard infusion over all hard limit events, $\left(\frac{H L_{n P G}}{H L_{T}}\right)$, for every month starting from August 2011 to August 2012

The general $\frac{H L_{n P G}}{H L_{T}}$ trend does not follow the plot $\frac{H L_{T}}{I_{T}}$ curve. The correlation coefficient for $\frac{H L_{T}}{I_{T}}$ and $\frac{H L_{n P G}}{H L_{T}}$ plots is 0.2551 , signifying a weak linear correlation. The $\frac{H L_{T}}{I_{T}}$ plot starts off high and decreases until December, then slightly increases until April. In May, $\frac{H L_{T}}{I_{T}}$ drops to its lowest and reaches its highest point in August 2012. The higher rates in August 2011, August 2012 and September 2011may suggest that this rate follows a seasonal trend that is higher in the late summer, but the study period is not long enough to make this conclusion. Plot $\frac{H L_{n P G}}{H L_{T}}$ increases in the month after the first two drug library updates (in September 222011 and March 13 2012); 
however decreases after the last update in June 212012.

A change in the drug library could be reflected in $\frac{H L_{T}}{I_{T}}$ and $\frac{H L_{n P G}}{H L_{T}}$ rates. A peak in the number of HL events following a drug library update may indicate a library with limits that are too strict; and a drop may indicate a library with ranges that are too wide or that is appropriate for the department. In Figure 3.7, the general increase in plot $\frac{H L_{n P G}}{H L_{T}}$ combined with the decrease in $\frac{H L_{T}}{I_{T}}$ in the first few months of the study period may indicate that nurses are learning the library and learning to use a non-PG infusion after hitting a hard limit as a shortcut rather than to set up a library infusion or that library limits are too strict such that the infusion cannot be setup in the library. Knowledge of the library may also account for the relatively low dips in September, March and June/July (dates corresponding to drug library updates) in $\frac{H L_{n P G}}{H L_{T}}$. The slight increase in the $\frac{H L_{T}}{I_{T}}$ in April (after a library update in March) followed by a decrease in May below the March levels could be indicating a learning curve: at first more hard limits are hit, but with time the clinicians adjust their practice to fit the library. A steady decrease in HL rate (plot $\frac{H L_{T}}{I_{T}}$ ) combined with a steady increase in plot $\frac{H L_{n P G}}{H L_{T}}$ as from September to December, could indicate that clincians are not well trained on the steps to take after a HL event which is why they setup a non-PG infusion more often after the event.

HL events may be caused by pump user errors (e.g., order entry errors), prescription errors and non-standard prescriptions. User errors, and prescription errors are unintentional actions that are therefore expected to occur at a steady rate unless a change is put in place (e.g., introduction of a new pump may increase pump user errors on all pumps or a system change aimed to reduce prescription errors will have a positive effect). Non-standard prescriptions may or may not be occurring at a steady rate, depending on patient needs, doctor prescribing habits and hospital policies. Overall, plot $\frac{H L_{T}}{I_{T}}$ is a fairly steady plot, ranging from 4 to $6 \%$ in the 13 month study 
period, as expected due to steady user and prescription error rates. Actions and steps taken by the user after hitting a HL event are intentional and this is reflected in the fluctuating plot $\frac{H L_{n} P G}{H L_{T}}$. A clinician may program a non-PG infusion after a HL event to enter a non-standard prescription outside of the library, or to setup up the infusion faster (due to frustration of the high number of steps or uncertainty of steps to take after hitting a hard limit) whether or not it was a standard prescription and the event signalled a user error.

Note that the number of cases for $H L_{n P G}$ is relatively low and results in low percentages when computing rates over all HL events and/or infusions. This accounts for the low percentage values for both plots in Figures 3.7 and 3.8. Note that $\frac{H L_{n P G}}{H L_{T}}$ and $\frac{H L_{T}}{I_{T}}$ have different scales and a variation in one plot is not necessarily equivalent to a variation in the other. 
$\frac{I_{n P G}}{I_{T}}$ and $\frac{H L_{T}}{I_{T}}$ rate plots by month:

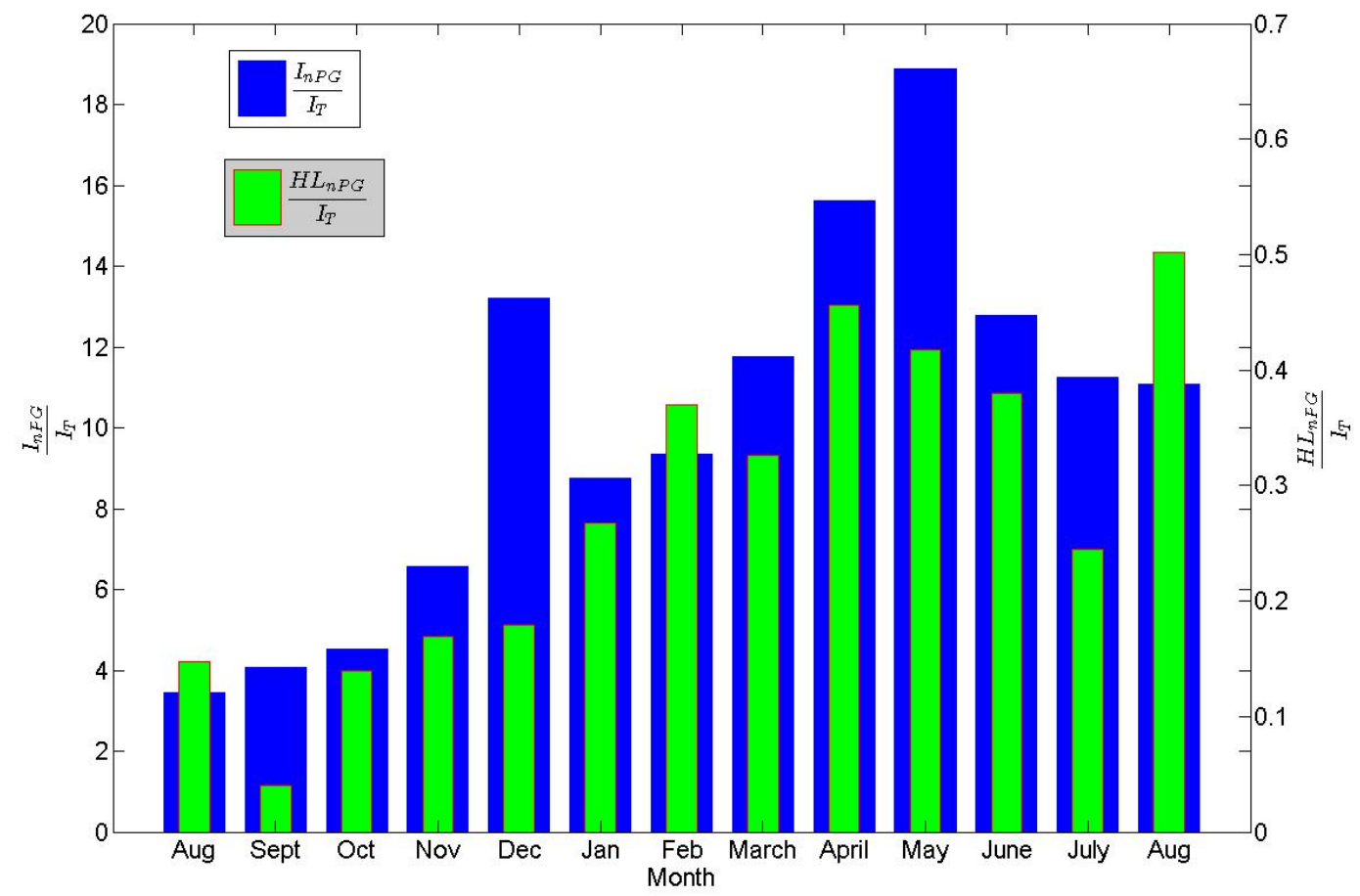

Figure 3.8: The percent of Hard limit events followed by a non-PharmGuard infusion, $\left(\frac{H L_{n P G}}{I_{T}}\right)$, and the percent of non-PharmGuard infusions over all infusions, $\left(\frac{I_{n P G}}{I_{T}}\right)$, from August 2011 to August 2012

Both plots $\frac{I_{n P G}}{I_{T}}$ and $\frac{H L_{n P G}}{I_{T}}$ are skewed to the right indicating the increase in $\frac{I_{n P G}}{I_{T}}$ and $\frac{H L_{n P G}}{I_{T}}$ trends until the last two months of the study, where both begin to decrease. The correlation coefficient for plots $\frac{I_{n P G}}{I_{T}}$ and $\frac{H L_{n P G}}{I_{T}}$ is 0.7478 , signifying a good linear correlation between both variables. Plot $\frac{I_{n P G}}{I_{T}}$ peaks in the month of May, two months after the second drug library update, and one month before the third drug library update. The Plot $\frac{I_{n P G}}{I_{T}}$ also peaks in December, and would be difficult to be linked to a drug library update. Plot $\frac{H L_{n P G}}{I_{T}}$ goes up for the first two drug library updates, and goes down for the last drug library update.

The strong correlation in plots $\frac{I_{n P G}}{I_{T}}$ and $\frac{H L_{n P G}}{I_{T}}$ is expected because a change in the 
number of non-PG infusions setup after a HL event will increase the overall number of non-PG infusions as well. An increase in HL events is an indicator that there may be issues with pump usage or the drug library. It is possible that the $\frac{I_{n P G}}{I_{T}}$ peak in May may have been what prompted the June 2012 library update. Both plots $\frac{I_{n P G}}{I_{T}}$ and $\frac{H L_{n P G}}{I_{T}}$ decrease in June and July which may be a positive indicator that the library updates deployed during March and June 2012 have helped to reduce the overall number of drug library workarounds and non-compliance. In August, the non-PG rate (plot $\left.\frac{I_{n P G}}{I_{T}}\right)$ remains the same while the $\frac{H L_{n P G}}{I_{T}}$ curve increases, suggesting that a larger fraction of non-PG infusions were prompted by a HL event, and reasons why this could be so should be investigated to determine if the library meets the needs of the clinicians and patients, if it is a workaround or acceptable practice. Setting up a non-PG infusion after a HL event is not proper pump use and is a case of bad practice if it is not done because of a non-standard prescription. Training on the significance of HL events and the steps to take after the event should be presented to educate clinicians on proper pump usage. If clinicians develop the habit of setting up a non-PG infusion after every HL event, then there is no way of knowing what the possible user error that the HL event caught was, if it was detected or corrected by the user. In time, this practice may also lead to clinicians directly setting up a non-PG infusion to avoid hitting a hard limit, which may be what would explain the drop in plot $\frac{H L_{n P G}}{I_{T}}$ and increase in plot $\frac{I_{n P G}}{I_{T}}$ (non-PG rate) in May.

Recall that the number of cases for $H L_{n P G}$ is relatively low and results in low percentages when computing rates over all HL events and/or infusions. Axis scales also vary for Figure 3.8 so that fluctuations in trends are not equivalent for both plots. 


\section{$\frac{I_{n P G}}{I_{T}}$ and $\frac{H L_{n P G}}{I_{n P G}}$ plot for drug library profiles:}

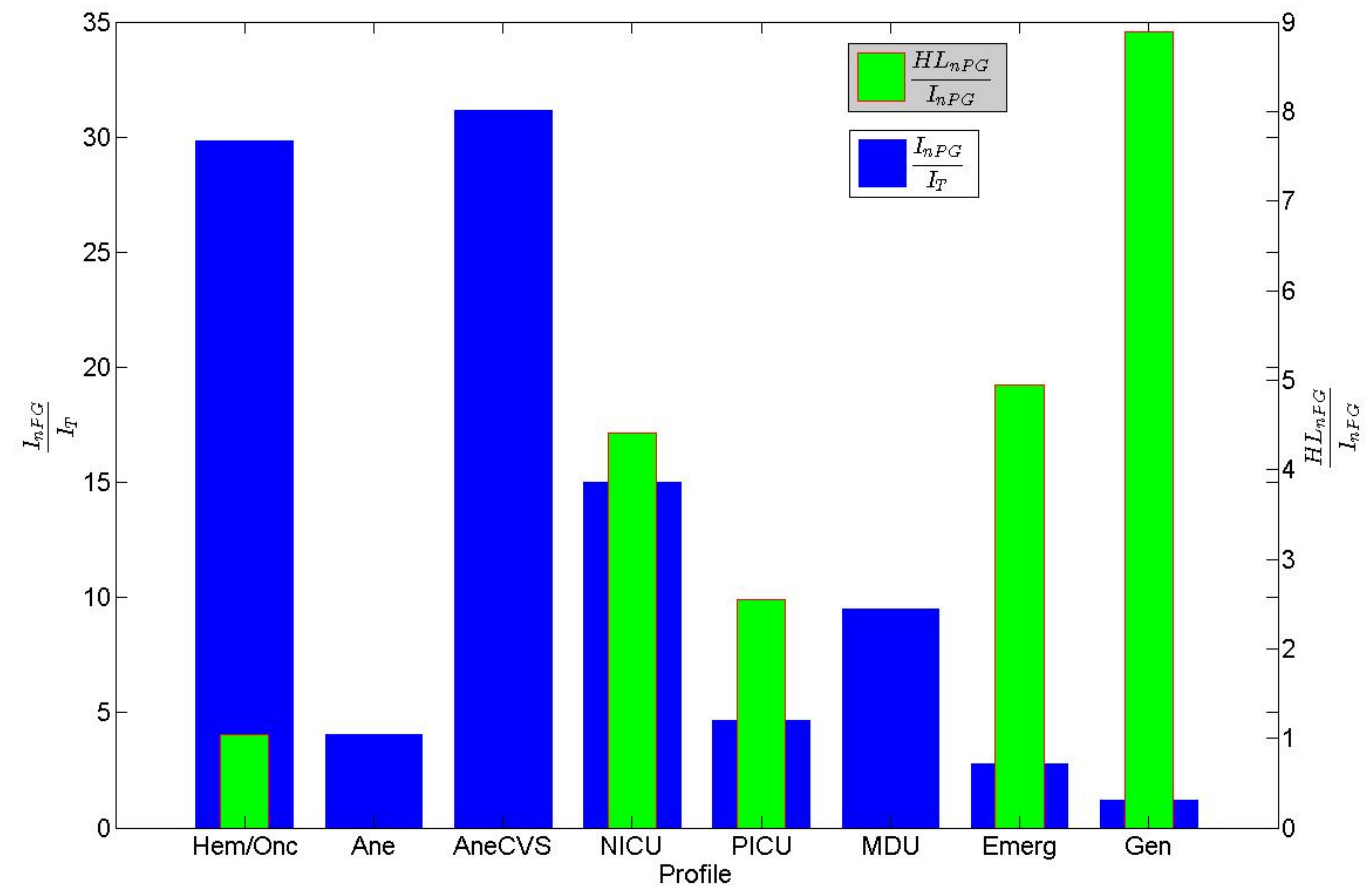

Figure 3.9: The percent of non-PG infusions over all infusion, $\left(\frac{I_{n P G}}{I_{T}}\right)$, and the percent of non-PG infusions that followed a Hard limit event, $\left(\frac{H L_{n P G}}{I_{n P G}}\right)$, for nine CHEO drug library profiles from August 2011 to August 2012.

Plot $\frac{H L_{n P G}}{I_{n P G}}$ shows that in only six out of nine profiles, a non-PG infusion is setup after a hard limit is reached, and that the General Peds/Surg profile has the most occurrences of this action.

In Figure 3.9, the General Peds/Surg (Gen) profile has the lowest rate of manual mode infusions. The Anaesthesia CVS (AneCVS) and Hem/Onc profiles have the highest rate of non-compliance, and therefore could be a good place to start for reducing overall non-compliance rates, first by reviewing library limits to find if libraries are insufficient and interviewing users of the library. Though the overall count for HL events followed by a non-PG infusion is low compared to other values, the 
profile plot shows where the $H L_{n P G}$ infusions are occurring, allowing to target an area of where this type of bypassing takes place.

\section{$\frac{I_{n P G}}{I_{T}}$ plot by month for the Hem/Onc profile}

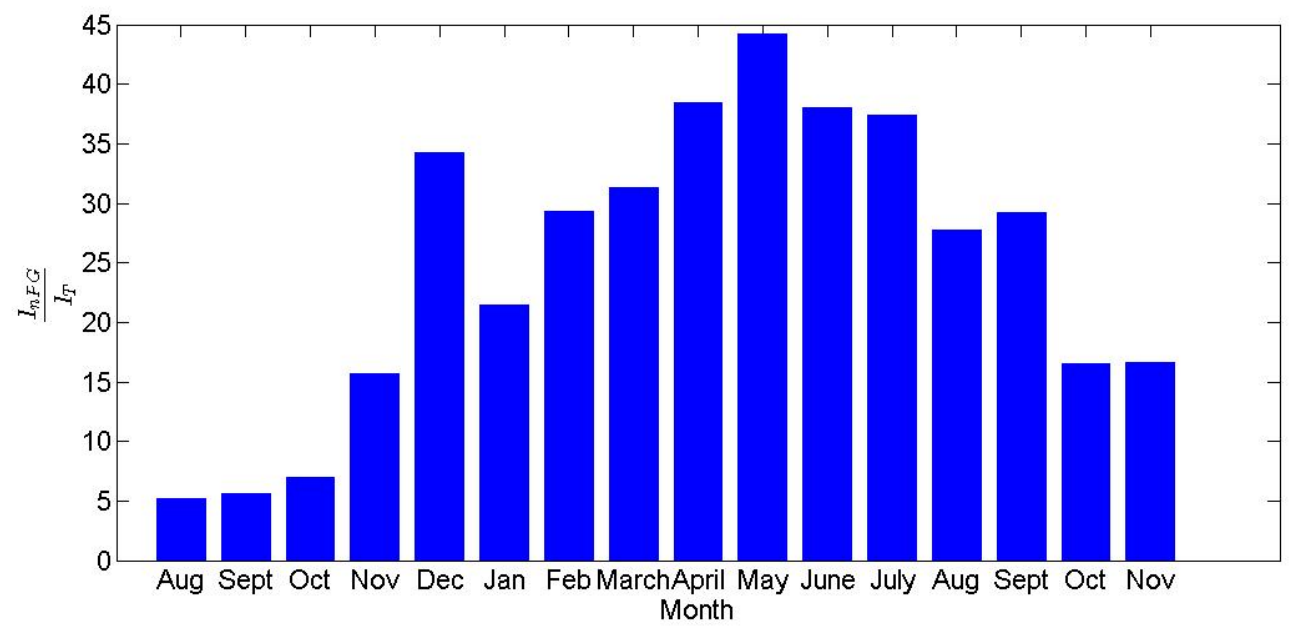

Figure 3.10: The percent of non-PharmGuard infusions over all infusions, $\left(\frac{I_{n P G}}{I_{T}}\right)$, by month for the Hem/Onc profile from August 2011 to November 2012

In Figure 3.10, it is apparent that the non-compliance trend increases for nearly every month until May. This increase was observed by the drug library manager at CHEO and discussed with the nurses at a nurse meeting in July. In August, a drop of $10 \%$ in non-compliance can be seen, then in October, another $10 \%$ drop. This is a positive indicator that shows evidence of the effectiveness of communication on compliance and infusion pump benefits. 


\section{$\frac{I_{n P G}}{I_{T}}$ plot by month for the Anesthesia CVS profile}

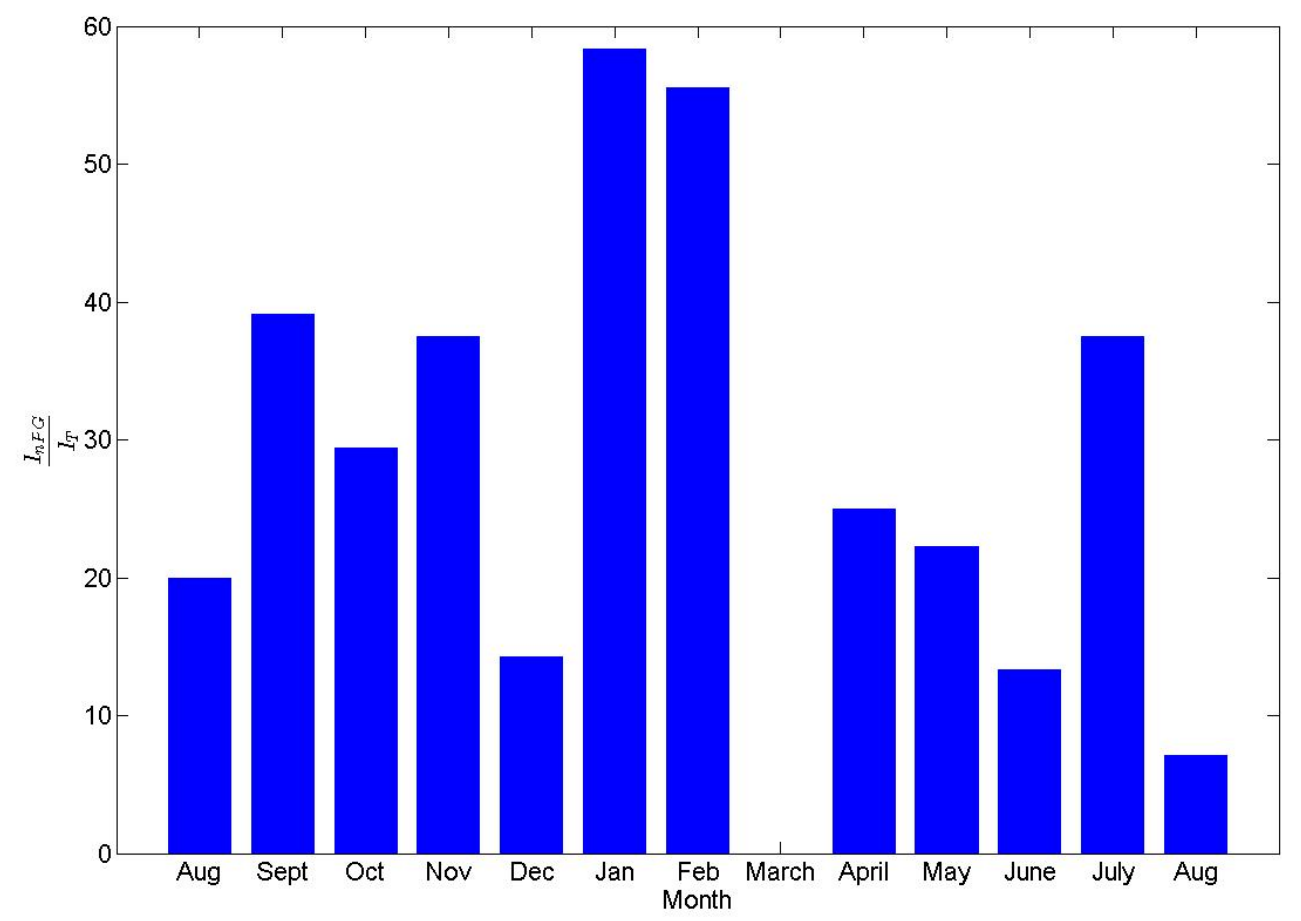

Figure 3.11: The percent of non-PharmGuard infusions over all infusions, $\left(\frac{I_{n P G}}{I_{T}}\right)$, by month for the Anesthesia CVS profile from August 2011 to August 2012

In Figure 3.11, the non-compliance ranges from $0 \%$ to around $58 \%$. In March, there were only 12 programmed infusions with that were all completed within the drug library, resulting in a 0\% non-compliance. The Anesthesia CVS high non-compliance values may be a results of the nature of practice applied by anesthesiologists. A unique feature of this profile is that the users of the pump are also the prescribers of the drugs. This is often not the case in other profiles and units. During surgery, the anesthesiologist is constantly monitoring the patient and accordingly adjusting medication levels based on patient responses. Non-compliance is an issue because it means that the pump is not able to detect pump programming errors; however, when a patient is being constantly monitored during surgery, any negative effects of 
an error would be apparent and detected by the clinicians in the operating room.

The Anesthesia CVS profile has been used by 3-4 anaesthesiologists at CHEO for the past few years. They were interviewed about the drop in compliance and any reasons for the change in rate and encouraged to offer any suggestions. The clinicians confirmed that there was no change in practice, no change in staff and no new medications in the drug library that would account for the fluctuation in the compliance rates. The compliance findings could not be explained.

\subsubsection{Conclusions}

Analysis of plots presented in the previous section led to the creation of several hypotheses that could lead to future work and research. A common factor in the hypotheses that could affect HL and non-PG trends plotted in the previous section are prescriptions made with non-standard dosing that would fall outside of drug library limits. Having a system in place that tracks non-standard prescriptions and when they are programmed in the pumps would greatly improve our analysis by giving a value to this unknown factor. More understanding of trends of non-standard prescriptions will enable focusing on analyzing hard limit events and non-PG infusions that are not associated with non-standard prescriptions and those that are separate groups. Ultimately, the aim in smart pump research is to increase compliance and eliminate hard limit events caused by intentional programming in order to improve patient safety. Other unknown factors and limitations are prescriptions and patient information. This would help identify the true hard limit event trigger, if it is a user error, prescription error or non-standard prescription.

The purpose of this section is to illustrate how a few measures from PharmGuard reports could be used to discover new hypotheses and research questions. In the data extracted, the number of HL events followed by a non-PG infusion is small (in Table 
3.4), and only represent a small fraction of HL events and infusions, which is a factor that does not allow us to make any definite claims, but only possible inferences with the collected data. 


\section{Chapter 4}

\section{Gentamicin Case Study}

\subsection{Introduction}

The objective of this chapter is to demonstrate how the smart pump database can be used to find ways to improve usability for reducing Hard limit events with quantitative analysis. Observing Hard limit event parameters and follow-up actions may give insight into factors that cause and lead to Hard limit events, which in turn can be used to revise pump training and drug libraries. A challenge in the analysis of infusion pump data is the large amount of data available. Drug usage differs for different drugs and different profiles because of the different library, users and patients; therefore, this chapter narrows down the data set to one drug and one profile. For this work, a case study was designed to follow one drug with a high hard limit rate, gentamicin, for a year (August 2011 to August 2012) in the General Peds/Surg profile.

For this case study, the General Peds/Surg profile was selected because it had the highest total infusions (1506) and total Hard limit event counts (132) for gentamicin. General Peds/Surg is the main profile used by CHEOs three largest inpatient units which encompass about $54 \%$ of the hospitals total inpatient bed capacity of 167 beds.

Gentamicin is an antibiotic used to treat respiratory, urinary tract, soft tissue or 
hard tissue infections [67]. The main risk of gentamicin administration is vestibular function damage leading to hearing loss and loss of balance. Renal function damage is also a risk, when combined with other drugs. Gentamicin is normally administered three times a day; however, a larger dose can be given once a day. Allowable dosage ranges are lower when it is administered multiple times a day. For this reason, gentamicin is listed twice in the library as: 1) "Gentamicin $5 \mathrm{mg} / \mathrm{mL}$ traditional" for up to three daily deliveries and 2) "Gentamicin $5 \mathrm{mg} / \mathrm{mL}$ once daily" for one delivery per day. Gentamicin is a weight-based drug that is prescribed based on patient weight. The limits for the traditional dose are $0.5 \mathrm{mg} / \mathrm{kg}$ to $3.5 \mathrm{mg} / \mathrm{kg}$, and are $4 \mathrm{mg} / \mathrm{kg}$ to $8 \mathrm{mg} / \mathrm{kg}$ for the once daily dose.

\subsection{Methodology}

For this case study, events that described one infusion were isolated from the Event History report in order to determine which actions were taken following a Hard limit event. Then, various types of actions that were taken following a Hard limit event were listed. Events describing one infusion were identified by grouping consecutive events that follow a logical pump setup sequence occurring on a single pump. A pump setup sequence will have a Setup event, Begin event, and an End event.

Infusion Details reports were generated from the PharmGuard database server for gentamicin in the General Peds/Surg profile, for the study period from August 2011 to August 2012. Hard limit events were isolated, using Microsoft Excel, along with the associated details on the pump serial number, date and time. For each Hard limit event listed in the Infusion Details report, an Event History report was generated for the associated pump on the day of the event to extract events following the Hard limit event. The events were categorized based on the actions following the Hard 
limit event and based on the type of error that caused the hard limit as determined based on the follow-up actions.

\subsubsection{Hard limit event follow-up actions}

The Hard limit events for gentamicin were found to be followed by several types of events that correspond to an action taken by the clinician:

1. Switched administration mode: Deliver an infusion with the other drug option. That is, if the hard limit was hit with the once daily option, the nurse reprogrammed the pump to infuse using the traditional option, and vice versa.

2. Manual mode: Deliver a non-PG infusion, by programming either the Volumeover-Time or flush category options for longer than 25 minutes from the General Peds/Surg profile. The flush option is designed to empty the infusion lines at a specified rate (usually at the same rate that the previous infusion was delivered), and should take a short amount of time to complete. The flush option can also be used to delivery a non-PG infusion, even if that is not its purpose or intended design.

3. Adjustment: To deliver the infusion, the dose value or weight were changed to be within the soft limits.

4. Adjustment with Soft limit override: Input a different value and triggered a soft limit event. The soft limit was overridden and the infusion proceeded.

5. Pump turned off: The pump was turned off.

6. Infusion of another drug: A different drug was infused. 


\subsubsection{Hard limit event error}

A major limitation in this study is the lack of information regarding patients and prescriptions; therefore, true error and any resulting patient harm cannot be determined from the data alone in this study. However, two types of user errors were inferred from analysis of the event details:

1. Selection error: The traditional option was selected instead of the once daily option or vice versa. If the wrong gentamicin option is selected, then the prescribed dose will result in a Hard limit event when entered.

2. Order entry error: The wrong key is entered, for example, a zero instead of a decimal point or vice versa.

3. Other: Not enough data to classify as selection or order entry error. Possibly not an error.

A selection error was found by comparing the dose value that exceeded the Hard limit event with the gentamicin dosing limits, for events classified under 'Switched administration mode', 'Manual mode' and 'Adjustment' actions where the weight was adjusted (rather than the dose) only. This required calculation of the limits based on patient weight entered. First, the weight was calculated based on the upper and lower hard limit values in the Hard limit event. Second, the limits were calculated for both the traditional and once daily options based on the calculated weight. Third, the dose that triggered the Hard limit event was compared to the newly calculated ranges to see what drug option was supposed to be delivered based on the entered weight and dose. If the Hard limit event happened for gentamicin traditional, and the dose was appropriate for a once daily infusion; or if the Hard limit event happened 
for a gentamicin once daily, and the dose was appropriate for a traditional infusion, then, the event was classified as a selection error. The following is an example:

A patient with a weight of $10 \mathrm{~kg}$ is prescribed $20 \mathrm{mg}$ of gentamicin three times a day. For a $10 \mathrm{~kg}$ patient, the limits for a three times daily dosing is $5 \mathrm{mg}$ to $35 \mathrm{mg}$, and for once daily dosing is $40 \mathrm{mg}$ to $80 \mathrm{mg}$. When a clinician programs the infusion and selects 'gentamicin 5mg/mL once daily', a Hard limit event is triggered. If the clinician goes back to select 'gentamicin $5 \mathrm{mg} / \mathrm{mL}$ traditional', to enter a new weight, or to program a manual mode infusion, then it is suggested that the clinician had initially selected the wrong drug option. The cases where the clinician goes back to enter a new weight in order to deliver the same dose entered are counted as selection errors in this study, but may be a situation where the wrong weight was initially entered or a workaround for delivering a dose outside of the limits.

An order entry error was found by comparing the dose value that triggered the hard limit alarm, and the re-entered value for events classified under 'Adjustment' and 'Adjustment with Soft limit override' actions. Cases when it appeared that there was a zero instead of a decimal point or vice versa, a decimal point in the wrong place or a missing/extra zero, then the event was classified as an order entry error.

Hard limit events classified in the 'other' category did not have enough data to classify as selection or order entry error. Some of these events may be selection errors that were not detected because the entered weight was different than the weight used to calculate the dosage. Some events may be a user error where a different number was entered instead of the dosage (e.g., unintentionally entering weight instead of dosage, or entering dosage from a different prescription order). Some of these events may account for the non-standard prescriptions ordered outside of the gentamicin hard limits. 
Analysis of consultations between the registered nurse, pharmacist or physician following a Hard limit event is beyond the scope of this review. Hard limit analysis in this report is evidence-based as it is leveraged by actual Medfusion data from CHEO.

\subsection{Results}

The total number of PharmGuard infusions (as determined by the number of PharmGuard infusion Begin events) in the General Peds/Surg profile within the study period was 36560. Table 4.1 presents the number of times each of the follow-up actions occurred. There were cases where the same dose that triggered the Hard limit event was re-entered, registering two, three, or four Hard limit events in a row; for these repeated Hard limit events, only the follow-up action for the last event of the series was used for classifying follow-up actions.

Table 4.1: Hard limit event follow up actions.

\begin{tabular}{cccc}
\hline Follow up actions & \multicolumn{3}{c}{ Gentamicin $5 \mathrm{mg} / \mathrm{mL}$} \\
& Traditional & Once daily & Total \\
\hline \hline Switch admin mode & 17 & 30 & $\mathbf{4 7}$ \\
Manual mode & 7 & 12 & $\mathbf{1 9}$ \\
Adjustment & 21 & 20 & $\mathbf{4 1}$ \\
Adjustment with soft limit override & 0 & 3 & $\mathbf{3}$ \\
Pump turned off & 2 & 2 & $\mathbf{4}$ \\
Infusion of other drug & 1 & 2 & $\mathbf{3}$ \\
\hline Total number of Hard limit events & 54 & 78 & $\mathbf{1 1 7}$ \\
Total number of Soft limit overrides & 0 & 17 & $\mathbf{1 7}$ \\
Total infusions & 836 & 670 & $\mathbf{1 5 0 6}$ \\
\hline
\end{tabular}

A look at the numbers in Table 4.1 reveals that the majority of hard limit cases 
Table 4.2: User errors.

\begin{tabular}{ccc}
\hline Error Type & Number of Cases & Percentage \\
\hline \hline Selection & 64 & $54 \%$ \\
Order Entry & 13 & $11 \%$ \\
Other & 40 & $34 \%$ \\
\hline Total & 117 & $100 \%$ \\
\hline
\end{tabular}

were Switch admin mode, Adjustment and Manual mode categories. The sum of the follow-up action cases in the table sum up to 117 Hard limit events, because of the 15 cases of repeated Hard limit events that were not counted. The total number of infusions corresponds to the number of gentamicin Begin events; it was not determined if the infusions were delivered.

Table 4.2 presents the number of user errors as suggested from analysis of the Hard limit events by considering dose and follow-up action.

All Switch admin mode cases were found to be selection errors, except for one case: a completed gentamicin infusion was followed by the Hard limit event and a three minute infusion; this suggests that the clinician set up the gentamicin infusion in the place of a flush to clear out the infusion lines. Thirteen out of nineteen manual mode cases, and five out of 41 adjustment cases appeared to be selection errors.

\subsection{Discussion}

Case study results show that when setting up a gentamicin infusion in the General Peds/Surg profile on the Medfusion 4000 pump, selecting the wrong drug option accounts for more than half of the Hard limit events (about $54 \%$ of the events). In the majority of those cases, 47 out of 64 , the clinician went back to select the appropriate 
drug option; however, in the remaining 17 cases, the clinician made a programming adjustment to deliver the dose. This finding is evidence that smart pumps introduce new types of errors not found in traditional pumps. The study results also show that $11 \%$ of the Hard limit events were order entry errors that the pump was able to catch, which is evidence of the value of the smart pump system. When all gentamicin infusions are considered (1506), a prevention rate of $0.9 \%(13 / 1506)$ can be estimated. The data would not have revealed prescribing errors, which may have contributed to Hard limit events.

Analysis of gentamicin Hard limit event follow-up actions and user errors showed that the drug library detected programming errors and that Hard limit events may be caused by wrong drug option selection for cases where the drug is listed several times under different labels (slightly modified labels). This finding emphasizes the need for the consideration of human-computer interaction and environmental factors when designing the pump as well as the drug library. A selection error may be intentional due to lack of training or negligence; the user did not know that there was a difference in the limits for both options, or the user did not know (or believe) that selecting the appropriate drug was necessary. A selection error may also be unintentional and caused by the confusion between the labels because they are nearly identical, and by the clinician failing to read the entire label.

Drug selection errors are a problem for a few reasons. Firstly, selection errors may not always lead to a Hard limit event if the dose limits overlap for the intended drug and the selected drug. In those cases, the Event History report will not have report with an accurate representation of the true events when what was infused to the patient compared to what drug was selected and recorded in the drug library. This will cause issues in retrospect when analyzing the data because there will be a mismatch between the true event parameters and the recorded parameters. Secondly, 
when a selection error is made, an order entry error may not be caught if the entered value falls within the limits of the selected drug and this puts the patient at risk. This is a problem if clinicians become too dependent on the drug library and do not double-check infusion parameters, or if they do a double-check but miss the error. Eliminating selection errors saves time because it will avoid extra steps after the Hard limit event caused by the error. Eliminating selection errors by improving labels, eliminate Hard limit events caused by wrong drug selection, and therefore reduce overall Hard limit events. The reduction of Hard limit events will reduce the number of steps for setting up an infusion and avoid frustrations of using the library due to poor usability; this in turn will have a positive impact on compliance. Issues with library usability, prolonged pump setup time, and the perception of meaningless Hard limit event alarms are all possible factors discouraging the use of drug libraries and leading to low compliance rates.

The remaining 40 gentamicin Hard limit events could be cases where the drug library was not sufficient. Further analysis of these cases would be required to discover the reasons that a hard limit was hit. These 40 cases may also be attributed to a few patients or a few clinicians only.

In Table 4.1, the four cases where the pump was turned off, and the three cases where another drug was infused, may be cases where the pump was being tested in the wrong profile. Training and testing of the pump is meant to be done in the Training profile. When training and testing is done outside of the Training profile, it is impossible to know based on the data. These cases represent a few of the possible infusions suspected to be training and testing scenarios.

To reduce Hard limit events, increased clinician training and education may be required for the pump with respect to selection of drug program options, such as, onscreen drug option interpretation and the importance of the selection of the proper 
drug option. Renaming the two drug delivery options is also a possibility to reduce confusion between drug program options that have the same drug name on the onscreen label (e.g., changing "Gentamicin $5 \mathrm{mg} / \mathrm{mL}$ traditional" to "Gentamicin 5 $\mathrm{mg} / \mathrm{mL}$ three times daily" or to "Traditional gentamicin $5 \mathrm{mg} / \mathrm{mL}$ "). This recommendation is relevant to other profiles and to other drugs in the library with more than one option (e.g., tobramycin). Training and testing for every library update that has a change of limits or drug names is an option for finding out if the change has an effect on usability of the pumps.

\subsection{Conclusions}

Overall, the case study on gentamicin Hard limit events shows the benefit of smart pumps and the importance of considering usability factors when designing a drug library to create an overall improved infusion system that maintains the benefits of error prevention while avoiding unwanted side effects like selection errors. Results show that smart pumps can catch order entry errors which are cases where drug errors could have occurred and were prevented. As a consequence, the smart pump also introduces other errors (e.g., selection errors) which cause additional time, effort and, ultimately, cost. Usability can potentially be a central issue that can prevent errors like selection errors, and may in turn improve compliance.

In this study, a mismatch between drug labels and drug intended to be infused, resulted in drug selection errors which generated a higher count of Hard limit events. This case study raises awareness towards drug selection errors, as well as other possible usability errors. The information gained supports that more similar case studies should be designed to gain more understanding of new smart pumps systems. 


\section{Chapter 5}

\section{Analyzing Infusion Data Using Decision Trees}

One of the goals of this research is to understand hard limit events and why they occur. The purpose of this chapter is to investigate the application of decision trees in identifying factors that are linked to infusions that are associated with a Hard limit event (HL infusions) and those that are not (NoHL infusions), not to find the actual cause. Identifying these factors may help to find characteristics of infusions associated with a hard limit event and leads for further investigating conditions under which they occur. Results show that the decision tree technique, as an automatic analysis method and knowledge discovery tool, seems to reveal information that appears sensible. Similar analysis can be done with other infusion types, other combinations of variables and other events such as device alarms. 


\subsection{Introduction to the infusion data research question}

Smart infusion pumps are designed to reduce user errors in programming by catching entered values that are lower or larger than limits pre-programmed by pharmacy. When this occurs for programmed hard limits, the user is required to enter a different value; the Hard limit event and the Begin event, detailing the infusion parameters (dose, drug, profile etc), are saved in the Events History report. The Event History report also saves infusion parameters for all infusions programmed into the pump. (For more information on the Event History report and events, see Section 3.4.4).

\section{$5.2 \quad$ Objective}

The purpose of analyzing infusion data using decision trees is not to find a root cause or classify infusions into the two HL and NoHL classes, but to discover important factors based on splits near the top of the tree. The decision tree is applied as a knowledge discovery tool in this chapter. In the previous chapter, Chapter 2.2, important factors (e.g., odor and spore print color) were found at the top of the tree; therefore, analyzing only the first few nodes at the top of the tree supports our goals for this chapter.

\subsection{Methodology}

To extract infusion pump data, Event History reports from August 2011 to August 2012 were generated from the PharmGuard software. Using MATLAB, the infusion parameters for HL and NoHL infusions were extracted and saved in a matrix. The 
events of interest are the Setup event which represents the initial step to programming an infusion, the Begin event, which represents the start of an infusion, and the Hard limit event which represents that a hard limit was exceeded. For all infusions, it was not checked that infusions were completed or delivered.

The infusion data set is used to build two decision trees using the MATLAB decision tree implementation; one using the gini criterion and one using the deviance criterion for making a split in the decision tree. The resulting trees are very large; therefore, only the first few nodes are analyzed in this section. The two trees were compared and the identified factors were analyzed to assess the possibility in contributing to a higher rate of Hard limit events.

\subsubsection{Infusion data description and criteria}

The infusion variables extracted from the Event History reports are listed in Table 5.3. The data type of the variables and details are also listed. There were two types of data variables in the data set: numerical variables and non-numerical or categorical variables.

The methodology for creating the final infusion data set that is used to build the decision tree is described in this section. To create the HL infusion sub data set, first, all Hard limit events were identified, resulting in a total of 3624 events, 3316 of which were PharmGuard infusions. The Hard limit events for non-PharmGuard infusions (308) were eliminated. Second, to identify the parameters of the infusion where the Hard limit event occurred, Hard limit events followed by a Begin event on the same pump were isolated. There were a total of 1898 hard limit events immediately followed by an infusion begin event (HL-begin event pairs). Hard limit events followed by other event types (e.g., other Hard limit events, any alarms, power off/on) were eliminated 
Table 5.1: Infusion parameters.

\begin{tabular}{|c|l|l|}
\hline Variables & Data Types & Details \\
\hline \hline Profile & categorical & Profiles based on hospital department \\
Drug & categorical & $300+$ drugs \\
Patient weight & numerical & Patient weight entered in kg \\
Dose & numerical & Dose value entered \\
Unit & categorical & Dose units \\
Rate & numerical & Rate as computed by the pump software \\
Time of day & numerical & Time of the day \\
Date & categorical & Day, month and year \\
Pump serial number & numerical & Pump identification number \\
\hline
\end{tabular}

from the data set, because finding the infusion parameters associated with those Hard limit events would require manual analysis of the excel reports.

To create the NoHL infusion sub data set, all Begin events were identified, resulting in a total of 103547 events. Begin events for non-PharmGuard infusions (1688 events) and that were not associated with a drug (10122 events) were eliminated. This reduced the NoHL infusion data set to 91737. Begin events do not save the profile parameter, so the profile information had to be extracted from the Setup event. Infusion Begin events with a Setup event right before it (on the same pump) were isolated so that the profile could be identified, this reduced the NoHL set to 58125 infusions identified (Setup-Begin event pairs). Again, finding the profile variable for Hard limit events that are not directly preceded by a Setup event would require more manual analysis of the data set. To make sure that the Setup-Begin infusion pairs were not part of an infusion that had a Hard limit event associated with it, cases were eliminated where there was a Hard limit event on the same pump ten minutes or less before the Setup event which reduced the NoHL set to 58005 cases. Ten minutes is 
assumed to be the shortest infusion delivered by a smart pump, but is not proven.

From the final subset of 1898 HL infusions and 58005 NoHL infusions, only continous infusions for weight-based drugs that had dose entered in mg were included for the final decision tree analysis. Excluded infusions were infusions that had dose saved in different units (3868) or no units (6168), bolus infusions (46), loading infusions (40), infusions that had body surface area instead of weight (353) or no weight (14747), and infusions from the training profile (15). The final data set used in decision tree analysis had 1269 HL infusions and 39771 NoHL infusions. Table 5.2 summarizes the inclusion and exclusion criteria.

In the final data set of filtered infusions, a total of 254 drugs and 184 infusion pumps were in the data set; therefore, drugs and serial numbers were eliminated as variables. Infusion pump serial numbers would not be beneficial as a factor because certain pumps will remain in one department, while others will migrate and one pump will have several users. The drug variable, as a categorical variable with many levels, would not reveal much information in the binary decision tree technique selected (e.g.,, having a split that divides the drugs into two groups does not give much insight considering the large number of drugs). The unit variable was eliminated as well, because only one common unit for dose was included in the final data set, which was the mg unit. Date and Time of day were variables eliminated as well to focus on drug and patient factors. 
Table 5.2: Inclusion and exclusion criteria for infusion data.

\section{Exclusion Criteria Inclusion Criteria}

1. Bolus infusions 1. Dose in $\mathrm{mg}$

2. Loading infusions 2. PG infusion with profile

3. Manual mode infusions 3. Weight-based drug

4. Training profile 4. Setup-Begin pairs and HL-Begin pairs only

\subsubsection{Infusion data decision tree criteria}

Two trees were built with the infusion data using the gini and deviance criteria. The stop criteria used was to build a tree where all nodes are pure; however, only the first few nodes of the tree were observed for influential factors. The list of variables used for the infusion gini and deviance trees (NoHL vs. HL infusions) is in Table 5.3:

Table 5.3: Infusion data variables used to build infusion decision trees for NoHL vs. HL infusions.

\begin{tabular}{|c|l|l|}
\hline Attributes & Data Types & Details \\
\hline \hline Profile & categorical & Profile selected based on CHEO department \\
Patient weight & numerical & Patient weight entered in kg \\
Dose & numerical & Dose entered in mg \\
Rate & numerical & Rate as computed by infusion pump \\
\hline
\end{tabular}




\subsection{Decision tree results for infusion data}

The decision trees built with the infusion data are shown in Figures 5.1 (gini tree) and 5.2 (deviance tree). The details on the number of cases for each class at each node and the percent over the total number of cases and over the number of cases in the node are shown in Tables 5.4 and 5.5.

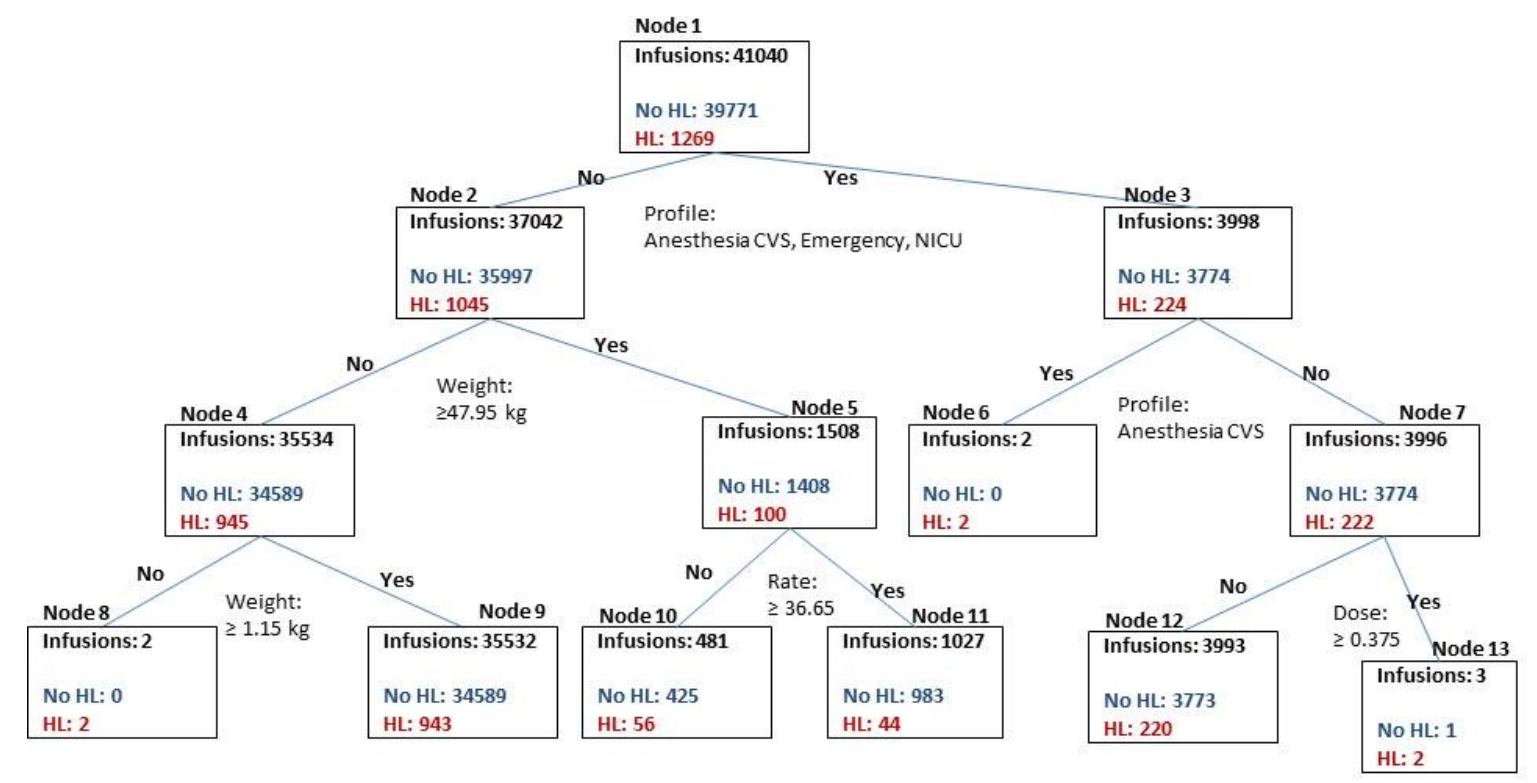

Figure 5.1: Infusion gini tree: Infusion tree built with the gini criterion 


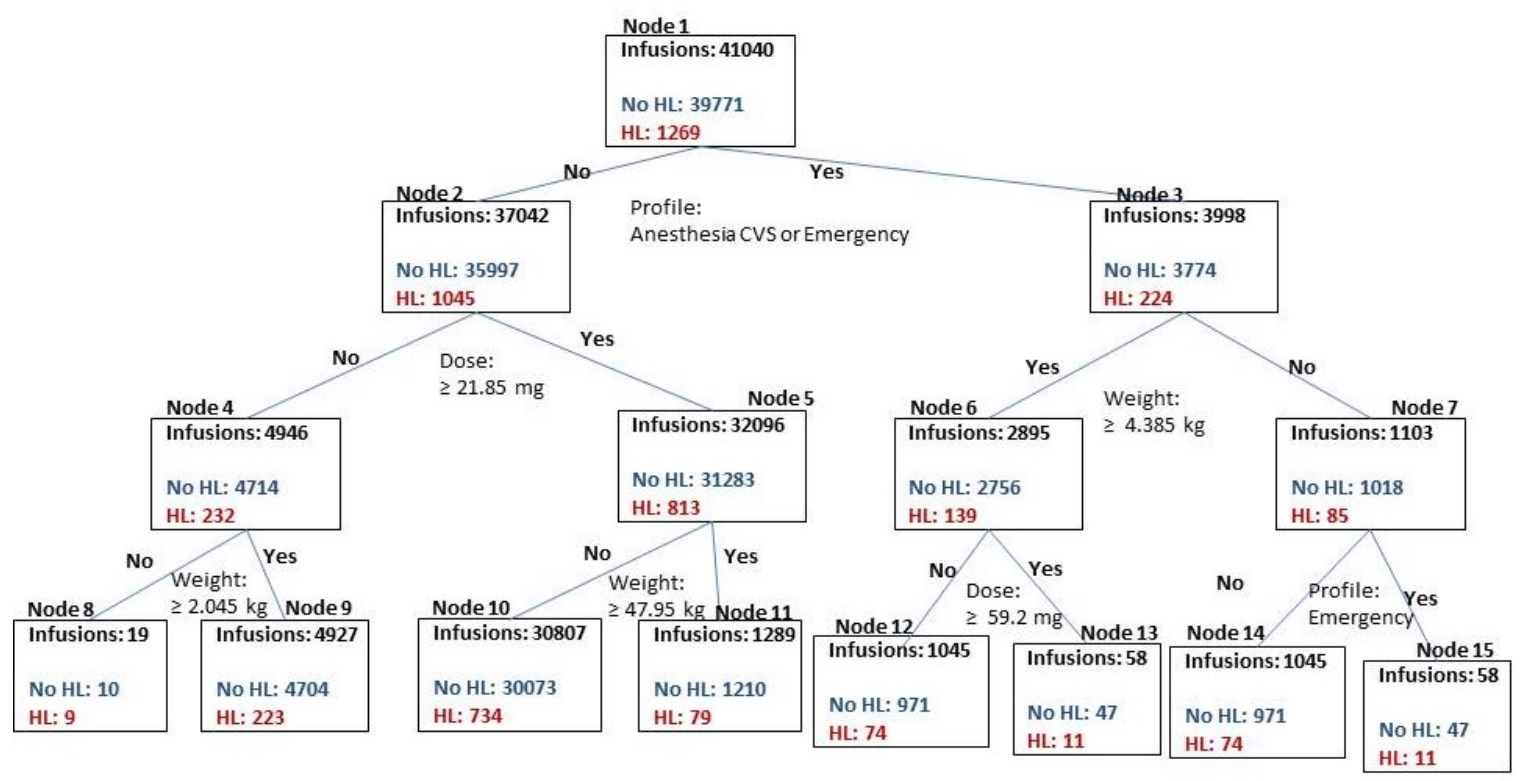

Figure 5.2: Infusion deviance tree: Infusion tree built with the deviance criterion 
Table 5.4: Infusion gini tree class count, percent of total and percent of node calculated at each node

\begin{tabular}{|c|c|c|c|c|c|c|}
\hline \multirow[t]{2}{*}{ Node \# } & \multicolumn{2}{|c|}{ Class Count } & \multicolumn{2}{|c|}{$\%$ of total } & \multicolumn{2}{|c|}{$\%$ of Node } \\
\hline & NoHL & HL & NoHL & HL & NoHL & HL \\
\hline 1 & 39771 & 1269 & 96.91 & 3.092 & 96.91 & 3.092 \\
\hline 2 & 35997 & 1045 & 90.51 & 82.35 & 97.18 & 2.821 \\
\hline 3 & 3774 & 224 & 9.49 & 17.7 & 94.4 & 5.60 \\
\hline 4 & 34589 & 945 & 87.0 & 74.5 & 97.3 & 2.66 \\
\hline 5 & 1408 & 100 & 3.54 & 7.89 & 93.4 & 6.63 \\
\hline 6 & 0 & 2 & 0 & 0 & 0 & 100 \\
\hline 7 & 3774 & 222 & 9.49 & 17.5 & 94.4 & 5.56 \\
\hline 8 & 0 & 2 & 0 & 0 & 0 & 100 \\
\hline 9 & 34589 & 943 & 87.0 & 74.3 & 97.4 & 2.65 \\
\hline 10 & 425 & 56 & 1.1 & 4.4 & 88 & 12 \\
\hline 11 & 983 & 44 & 2.5 & 3.5 & 96 & 4.3 \\
\hline 12 & 1 & 2 & 0 & 0 & 33 & 67 \\
\hline 13 & 3773 & 220 & 9.49 & 17.3 & 94.5 & 5.51 \\
\hline
\end{tabular}


Table 5.5: Infusion deviance tree class count, percent of total and percent of node calculated at each node

\begin{tabular}{|c|c|c|c|c|c|c|}
\hline \multirow[t]{2}{*}{ Node \# } & \multicolumn{2}{|c|}{ Class Count } & \multicolumn{2}{|c|}{$\%$ of total } & \multicolumn{2}{|c|}{$\%$ of Node } \\
\hline & NoHL & $\mathrm{HL}$ & NoHL & HL & NoHL & HL \\
\hline 1 & 39771 & 1269 & 96.91 & 3.092 & 96.91 & 3.092 \\
\hline 2 & 35997 & 1045 & 90.51 & 82.35 & 97.18 & 2.821 \\
\hline 3 & 3774 & 224 & 9.49 & 17.77 & 94.4 & 5.60 \\
\hline 4 & 4714 & 232 & 11.9 & 18.4 & 95.3 & 4.69 \\
\hline 5 & 31283 & 813 & 78.7 & 64.6 & 97.5 & 2.53 \\
\hline 6 & 2756 & 139 & 6.93 & 11.0 & 95.2 & 4.80 \\
\hline 7 & 1018 & 85 & 2.6 & 6.8 & 92 & 7.7 \\
\hline 8 & 10 & 9 & 0.03 & 0.7 & 50 & 50 \\
\hline 9 & 4704 & 223 & 11.8 & 17.7 & 95.5 & 4.53 \\
\hline 10 & 30073 & 734 & 75.6 & 58.3 & 97.6 & 2.38 \\
\hline 11 & 1210 & 79 & 3.0 & 6.3 & 94 & 6 \\
\hline 12 & 1703 & 64 & 4.3 & 5.1 & 96 & 3.6 \\
\hline 13 & 1053 & 75 & 2.7 & 6.0 & 93 & 6.7 \\
\hline 14 & 971 & 74 & 2.4 & 5.9 & 93 & 7.0 \\
\hline 15 & 47 & 11 & 0.12 & 0.87 & 81 & 19 \\
\hline
\end{tabular}


In both trees, the factor used to split the infusions at node 1 was Profile. For the Emergency, NICU and Anesthesia CVS profiles, HL represented 5.6\% ( $\mathrm{n}=224)$ of infusions; while for the General Peds/Surg, Hem/Onc, and MDU profiles, HL represented $2.8 \%(n=1045)$ of infusions. Note that the split in node 3 of the gini tree shows that in the data set, there are only 2 infusions in the Anesthesia CVS profile (node 6), and the remaining 224 infusions are in the Emergency and NICU profiles. Another split that is common to both trees is the weight split at $47.95 \mathrm{~kg}$ at node 2 in the gini tree and at node 5 in the deviance tree for infusions with a dose over $21.85 \mathrm{mg}$. In the gini infusion tree, for patients over $47.95 \mathrm{~kg}$, HL rate was $6.6 \%$ (100 infusions); and was 2.7\% (945 infusions) for patients under $47.95 \mathrm{~kg}$. For the deviance tree, HL rate was 6\% (79 infusions); and was 2.38\% (734 infusions) for patients under $47.95 \mathrm{~kg}$. In both trees, the splits had more than double of HL infusions for a weight over $47.95 \mathrm{~kg}$ than for the weight under $47.95 \mathrm{~kg}$; which highlights the significance of the weight factor.

Also for the gini tree, node 5 splits the HL infusions based on rate into 56 infusions into node 10 and 44 infusions into node 11 . Here, HL infusions represent $12 \%$ of infusions in Node 10 and $4.3 \%$ of infusions in node 11, which is more than double. Node 6 is a pure node, but only contains two infusions; therefore, the factor differentiating node 6 and node 7 is ignored for this analysis.

In the deviance tree, there were no other splits that divided the HL infusions in a 2:1 ratio between the two children nodes. However, it may be worth noting that weight is a factor in 3 of the 7 splits, which may be more evidence that weight is a significant factor. 


\subsection{Discussion}

The decision tree analysis suggests that Profile is the primary factor in predicting HL infusions. Specifically, Emergency and NICU profiles have nearly double the HL rates compared to the other four profiles combined. In the other profiles (General Peds/Surg, Hem/Onc, MDU, and PICU), patients that have a weight higher than $47.95 \mathrm{~kg}$ have double the chance of having a HL infusion, and particularly those infused with a dose larger than $21.85 \mathrm{mg}$. The decision tree method is known to be a brittle technique; that is, the decision tree may vary if a small portion of the data is changed or left out. The consistency in the gini and deviance trees suggests some consistency of the reported results.

An increased Hard limit event rate associated with a particular factor may also indicate that there is a higher rate of clinical errors in general that are also associated with that factor. For instance, a higher Hard limit event rate for Emergency and NICU profiles, may also mean a higher rate of errors in those areas, which further justifies the use of smart pumps for catching potential errors. In fact, further research on pediatric medication errors in the Emergency and NICU, has revealed findings suggesting that the conditions of work and environment in the Emergency department, and the patient profiles in NICU make that the departments provides several opportunities for error. A study at CHEO analyzed prescriptions using patient record and compared to hospital standards of practice found that the NICU had the highest rate of discrepancies at $7.49 \%$ of prescriptions [68]. Discrepancies in the study refer to incomplete prescriptions, wrong dose, wrong frequency, illegibility, wrong rate, wrong route of administration or wrong drug. A summary on the analysis

of errors in the emergency department and NICU is summarized in Sections 5.5.1 and 5.5.2, respectively. 
The high Hard limit event rate associated with large weights (higher than 48 $\mathrm{kg}$ ) could be due to two general explanations. The first explanation is that the dose is prescribed based on the patient weight, as should be normal practice for pediatric patients, which results in a dose that is above the limit for adult patients. In other words, the patient is wrongly prescribed a dose that is above the adult limit. As discussed in Section 3.2, a unique and challenging element to the pediatric patient population is that a large number of drug dosing limits are weight-based, whereas most drugs are not weight-based for the adult population but still have a maximum dosage, time or rate limit that must not be exceeded. In these situations, the prescribing doctor followed the standard prescribing procedures; however, may have made an unintentional order error. The second explanation is that a syringe pump was the wrong device to use for the prescription. Recall that the Medfusion 4000 holds a maximum syringe size of $60 \mathrm{~mL}$; therefore, it has a limit to how much dose it can hold for any drug. Intuitively, a larger (or adult) patient would require a larger dose that may require either the use of a large-volume pump (that holds more than $60 \mathrm{~mL}$ ), a refill of the $60 \mathrm{~mL}$ syringe if the syringe pump is used, or mixing the dose in a higher concentration and lower volume to fit in the syringe. Using the second or third options presents the opportunity for error in mixing, and in calculating the dosages that would contribute to a rise in Hard limit events. For other explanations and to verify the proposed explanations would require further investigation and analysis on the infusions with a patient weight higher than 47.95 $\mathrm{kg}$ than completed in this chapter. 


\subsubsection{Analysis of errors in the emergency department}

A study conducted by an American hospital network, called the Pediatric Emergency Care Applied Research Network, data from 21 pediatric emergency departments aiming to identify characteristics for emergency departments in terms of patient safety and clinicians' perceptions of safety [69]. The results of the study showed that overcrowding, when there is not sufficient resources for the demand of emergency services, was a relevant issue and correlated with staff's perception of a safe environment. Long shifts (12 hours) and a high number of hours per week (80 to 96 hours per week), were conditions leading to fatigue and were normal practice. Both characteristics are factors affecting patient safety according to studies by [70] and [71]. In particular, a study on the effect of overcrowding on medication errors found an increased frequency of error with increased crowding [72]. Overcrowding and fatigue may place more pressure and distractions on the clinicians with a lower ability to respond in a high-stress environment and, therefore, create a challenge when interfacing with various medical devices, including the smart pumps, considering the steps required to

program it. An observation study testing the hypothesis that increased interruptions created more opportunity for error, found that there was a significant association between interruption frequency and procedural failures and clinical errors [73]. The Emergency and NICU departments may be departments with frequent interruptions occurring. Only 18 of the 21 departments studied used standard concentrations for infusions, which is the basis of how smart infusion pumps work to reduce error. Nonstandard prescriptions are a possible explanation for the relatively higher HL infusion rate in those departments. One study researching the variables associated with medication errors in pediatric emergency departments found that sicker children are more prone to errors [74]. Sicker children in the emergency department put the clinicians in a pressure situation, affecting ability to work efficiently with medical devices, and 
may also require prescriptions outside of pump limits. Also, since pediatric dosing is weight-based [75], it is possible that clinicians tend to estimate weight for sicker children during emergency care if administering medication in a timely manner is a factor in safe and urgent treatment. Weight-based dosing requires calculation of dose, which is another avenue for committing an error. One study administered a dose calculation test to 64 pediatrician trainees over two rounds that were four years apart in order to determine if pediatric trainees needed to be tested for dose calculation skills. The result was that 28 of the 64 students made more than one error on the test [76]. Errors in calculation may be detected by the smart pumps; however, this may not always be the case. A recent study from 2012 reviewed incident reports across 18 emergency departments, found that $39 \%$ of medical errors were errors in dose; often due to calculation errors and use of the wrong weight [10]. In general, errors in the emergency department have been found in all stages of care and personnel in the department [77]; fortunately, $98 \%$ of errors did not result in a significant adverse outcome in that study.

\subsubsection{Analysis of errors in the neonatal intensive care unit}

A study in the NICU on adverse conditions due to diagnostic procedure or treatment by a clinician, found a higher rate of incidence for younger and smaller children; rates were $3 \%$ at term and $57 \%$ at ages of 24 to 27 weeks [78]. In another study, patients less than 28 weeks old and born at a weight less than $1500 \mathrm{~g}$ had higher rates of adverse events [79]. These studies support findings on increased exposure to error with decreasing age. Patients in the NICU are very small and are still developing, even compared to older babies and children; in addition, their size, weight and other pharmacokinetic factors are changing at a relatively rapid rate. For these reasons, medication limits are narrower, and may be why it is easier to exceed these limits 
if standard dosing is not used for prescriptions or if a calculation error is made. In general, the NICU is a high-risk area of the hospital some environmental factors shared with the emergency unit: quickly changing patient state/acuity, several distractions, and unpredictable work flow.

\subsubsection{Contribution to trigger-based research}

A portion of research in general and pediatric medication errors is aimed at developing methods for identifying conditions or factors called triggers that are linked to medication incidents and errors [79-81] . A trigger is defined as "occurrences, prompts, or flags found on review of the medical record that 'trigger' further investigation to determine the presence or absence of an adverse event" by [80]. The goal is that these triggers could be used to create awareness and focus training on issues that the research has shown may lead to errors and patient harm. Similarly, decision trees can be used as a method for identifying triggers that are characteristic of HL infusions and that lead to further investigation of smart pump system components (e.g., clinician's practice/habits, training, or drug library). The decision tree technique is a method that can be explored for trigger-based research.

\subsection{Conclusions}

The results of the decision tree highlights profile and weight to be influential factors differentiating between NoHL and HL infusions. There are previous works that suggest that weight is a factor in medication errors in pediatrics and that characteristics present in unique hospital departments such as emergency and intensive care units create an environment that is prone to errors. The findings from the decision tree analysis are supported by the literature review. Based on literature review findings, 
NICU and emergency departments are high-risk complex environments with patients requiring special needs. Distractions, patient changing state, and especially size and weight are recurring themes for both departments that have been linked to high error occurrence. Hard limit events in smart pumps can be caused by a non-standard dose, from estimating patient weight, from a calculation error; or caused by user error from distractions, or misuse of pump (e.g., ending an infusion early and setting up a new one with the dose that is left). Decision tree findings may help inform clinical practice and procedures, by identifying influential factors and ordering them based on degree of differentiation. 


\section{Chapter 6}

\section{Summary}

The findings of this research showed the value in the smart pump system in improving patient safety, and the value in analyzing the smart pump data collected from the pumps. Infusion pumps are an old technology that is continuing to develop and improve. The research of infusion pumps requires collaboration between all stakeholders and researchers to gather a complete understanding of the system, and is necessary for ultimately reaching our goals of providing better health care. Conclusions from this research are summarized in Section 6.1, and recommendations for future work are listed in Section 6.2.

\subsection{Conclusions}

The analysis of compliance, Hard limit event and infusion data saved by the pumps could play a significant role in the improvement of smart infusion pumps. Comparing the trends of compliance and Hard limit events over time and around drug library updates presented possible evidence of the effects of drug library updates. Analyzing compliance by profiles showed that drug library updates and training could be targeted based on problem areas. The compliance increase by nearly $20 \%$ after training 
in July 2011 was evidence of the positive impact of the training. Similar process of analysis can be used to motivate training and library updates; and to provide evidence on the effects of interventions and changes.

The gentamicin case study was an analysis of hard limit events for 'Gentamicin 5 $\mathrm{mg} / \mathrm{mL}$ traditional' and 'Gentamicin $5 \mathrm{mg} / \mathrm{mL}$ once daily' and follow-up actions to identify user errors. Results showed the value of the smart pump in detecting user errors which were potential drug errors that may have harmed the patients treated. The study identified selection errors in more than half of the Hard limit events. Selection errors are user errors where the clinician selects and confirms the wrong drug option, when the drug is listed more than once in different forms. This work emphasizes the consideration of drug library design, especially the choice of drug labels. Elimination of selection errors would save time for a clinician programming the pump and also greatly reduce the Hard limit event count for gentamicin and other drugs listed more than once with similar labels.

The research presented on the decision tree is an example of a method that has potential of contributing to one of the infusion pump clarion themes defined at the AAMI/FDA infusion safety summit: Standardize systems and processes for reporting, aggregating, and analyzing infusion device incidents [44]. Researching various data analysis and trigger-based methods to apply to collected infusion data, such as the case study and decision tree technique tested in this thesis, allows to develop new tools for improving our knowledge of the system. The contributions in this thesis provide evidence on the benefit of smart pumps, and analyzing smart pump data; also, proposing and demonstrating the use of two techniques for infusion data analysis.

The decision trees were analyzed in this thesis to identify important factors that were associated with infusions that hit a Hard limit event and infusions that did not. First, the decision tree technique was applied to a mushroom data set to show that 
important factors that separated the two classes of the data set were identified at the top of the tree. Second, the decision tree was applied to the infusion data and the factors in the top of the tree were analyzed. Profile and weight were found to be important factors. Emergency profile and NICU were found to have double the hard limit events than the other four profiles (PICU, General Peds/Surg, Hem/Onc, MDU). A literature review of errors in the emergency and NICU profiles showed that errors are commonly found, which supports that the profile factors identified were sensible based on the assumption that a high error rate corresponds to a high Hard limit event rate. The second factors identified was weight; where a weight over $47.95 \mathrm{~kg}$ had double the Hard limit events than a weight under $47.95 \mathrm{~kg}$. This could be attributed to wrongly prescribing a weight-based dose that is above the adult limit, or errors with delivering a large dose using the $60 \mathrm{~mL}$ syringe pump. Further investigation of those factors and explanations would provide proof of the importance of those factors.

\subsection{Future work and recommendations}

In the data used for analysis in all forms of investigations (e.g., the compliance trends, the case study, the decision tree) used in this thesis, data on patient information and prescriptions would have greatly improved analysis. Among other things, this missing data would distinguish between Hard limit events caused by user errors or nonstandard prescriptions, and patient weight can be verified. CHEO's first electronic patient records system began its first phase of implementation in 2012, which is a step forward towards creating the network connecting infusion devices, patients and prescriptions. 
A method for making the distinction between hard limit events caused by nonstandard prescriptions, and hard limit events caused by user error in the PharmGuard database would greatly improve analysis of events. This method could also improve workflow by eliminating the need for the nurse to seek re-verification of the prescription when a hard limit is hit. This may mean designing a separate library that is altered by pharmacy based on non-standard prescriptions received and that is used only for those non-standard prescriptions when indicated on the order form. This library may be locked with a password that is written on the order form. An issue with this method is the limitation in the process of library updates, which is not always timely.

Future work suggested based on findings of this research is listed below:

1. Future work for the gentamicin case study

(a) Further analysis on the 40 other cases that were not classified as a user error. This may reveal a pattern that could pinpoint a repeated error. Perhaps find a weight range or dose range. Any information may help improve the library.

(b) Design and complete an identical case study after a targeted training or drug library change addressing the selection error (e.g., change in drug labels for gentamicin): Results would allow a comparison of Hard limit event counts, and user errors before and after the change to assess the effectiveness of the new drug library and/or training.

(c) Design and complete an identical case study for another drug that has more than one drug options in the drug library: This investigation 
would reveal if selection errors are a problem for other drugs. This could be for another drug with two administration options (traditional vs. once daily) or a drug that can be delivered in more than one concentration.

\section{Future work for decision trees}

(a) Apply decision tree testing to address "brittleness" of decision trees: The decision tree method is known to be a brittle technique; that is, the decision tree may vary if a small portion of the data is changed or left out. It is recommended to bootstrap samples of the data set to build a tree from each sample and look for consistency of features.

(b) Apply the same technique for $\mathrm{HL}$ and NoHL infusions with different variables: Using more variables will generate a new tree that may reveal the same important factors or lead to the discovery of new factors. Variables recommended to explore are: season, period of day, holiday (yes or no as a binary variable), and contextual variables such as crowding, ratio of users to patients and the time in the user's shift.

(c) Apply the same technique for HL and NoHL infusions with different inclusion criteria: Investigating different infusion types would identify new factors relevant to different infusions. Infusion types recommended: different units of dose, bolus infusions, loading infusions, infusions of a specific drug or groups of drugs. This would allow identification of new factors of influence and triggers that could lead to future research. 
(d) Apply the same technique for other classes of events or infusions: Instead of HL infusions, infusions that hit a soft limit can be used instead or used as a third class of infusions. Order entry errors and selection errors can be used as two class types and the decision tree can be used to find factors that influence or that are associated with one error or another.

(e) Design an automatic decision tree application for infusion data: The automatic decision tree analysis application with a user-friendly interface would allow the selection of infusion types and parameters with the use of checklists that will automatically build a decision tree and output a list of factors in order of importance. The actual tree would be hidden from the user, but the factors are of interest to the user and are what is presented. This application could be interfaced with PharmGuard software to allow automatic extraction of infusions.

(f) Investigate the use of a different decision tree technique: Investigating different decision tree techniques may also reveal new factors. In particular, a technique that uses more than one variable per split or produces more than two children.

(g) Investigate use of other techniques: Other data mining approaches should be tested on the infusion data. The technique should be suitable for categorical data. Two possible methods to be explored are the association rules and clustering methods. Association rules is a technique that measures the relationship between variables and is normally used for large databases, which is the case for the infusion data. The k-modes clustering 
method using weighted dissimilarity is suggested as an option. This clustering method uses k-modes rather than k-means to determine cluster centers, and is based on the ratio of frequency of attribute values in the cluster and in the data set [82]. Manual analysis can also be explored: plotting probability graphs for each category level (for categorical data) or for various ranges of variables (for numerical data) and observing the trends.

3. Future work for investigating relevance of factors identified by the decision tree technique

(a) For future research, interviews of emergency department staff at CHEO to find characteristics of overcrowding, work shifts, patient weighing, calculations and use of standard concentrations; and interviews of NICU staff at CHEO for characteristics of standard dosing and distractions. Linking patient records to infusion pumps would provide more knowledge on errors and allow to determine harm that errors may have caused and to compare to a study in the emergency department that found $98 \%$ of errors did not result in a significant adverse outcome [77].

General recommendations for smart pump research are listed below:

1. Drug library research: Drug libraries are a major component of the smart pump that influences hard limit events and compliance rates; therefore, future research in that area of smart pump design is recommended. A recommendation 
for reducing user errors related to drug library usage is to test the drug library with the new changes at every drug library update. Research is recommended to study the impact of delays in library update activation in pumps on the Hard limit event trends.

2. Link pumps to patient and prescriptions: The device server may contain false data if an improper weight, dose or drug is entered to intentionally bypass the library. This is a major limitation of research in smart pumps. Linking pumps to patients and prescriptions would allow one to isolate those cases for further investigation as well as aid in true error classification. Also, tracking cases where there is a clinical need to go outside of the limits would improve analysis of cases of non-compliance.

3. Plan a method of drug library testing and/or training: Drug library testing would help determine if a drug library change would improve or pump usage. This may be difficult to produce a test setting that is comparable to a real world setting. The motivation in programming the pump during a test setting would be different than the motivation in a real life situation, which could alter the results. 


\section{List of References}

[1] "Deaths associated with medication incidents: Learning from collaborative work with provincial offices of the chief coroner and chief medical examiner," ISMP Canada Safety Bulletin, vol. 13, no. 8, pp. 1-7, Aug. 2013.

[2] "Smart pumps are not smart on their own." [Online]. Available: https://www.ismp.org/newsletters/acutecare/articles/20070419.asp. [Accessed: 23-Mar-2014].

[3] M. Minelli, Big data, big analytics: emerging business intelligence and analytic trends for today's businesses. Hoboken, New Jersey: John Wiley \& Sons, Inc, 2013.

[4] R. Akerkar, Ed., Big data computing. Boca Raton: CRC Press, 2014.

[5] Q. Shaath, A. D. C. Chan, D. Nussbaum, K. Greenwood, M. MacNeil, R. Vaillancourt, and C. Ma, "A case study: gentamicin hard limit events and follow-up actions in smart infusion pumps," presented at the Canadian Medical and Biological Conference/ Association des physiciens et ingenieurs biomedicaux du Quebec, Hampton Inn \& Conference Centre, Ottawa, Ontario, 2013.

[6] L. T. Kohn, J. Corrigan, and M. S. Donaldson, To err is human building a safer health system. Washington, D.C: National Academy Press, 2000.

[7] I. of Medicine (U.S.). Committee on Quality of Health Care in America., Crossing the quality chasm: a new health system for the 21st century. Washington, D.C: National Academy Press, 2001.

[8] J. I. Westbrook, M. I. Rob, A. Woods, and D. Parry, "Errors in the administration of intravenous medications in hospital and the role of correct procedures and nurse experience," BMJ Quality \& Safety, vol. 20, no. 12, pp. 1027-1034, Jun. 2011.

[9] A. Szczepura, D. Wild, and S. Nelson, "Medication administration errors for older people in long-term residential care," BMC Geriatrics, vol. 11, no. 1, p. 82, 2011.

[10] K. N. Shaw, K. A. Lillis, R. M. Ruddy, P. V. Mahajan, R. Lichenstein, C. S. Olsen, J. M. Chamberlain, and for the Pediatric Emergency Care Applied Research Network, "Reported medication events in a paediatric emergency research network: sharing to improve patient safety," Emergency Medicine Journal, vol. 30, no. 10, pp. 815-819, Oct. 2012. 
[11] H. C. Government of Canada, "Patient Safety - Health Care System - Health Canada," 26-Jul2004. [Online]. Available: http://www.hc-sc.gc.ca/hcs-sss/qual/patient_securit/index-eng.php. [Accessed: 25-Nov-2013].

[12] Anne Elixhauser and Pamela Owens, “Adverse drug events in U.S. Hospitals, 2004,” Agency for Healthcare Research and Quality, Statistical Brief \#29, Apr. 2007.

[13] D. W. Bates, N. Spell, D. J. Cullen, E. Burdick, N. Laird, L. A. Petersen, S. D. Small, B. J. Sweitzer, and L. L. Leape, "The costs of adverse drug events in hospitalized patients. Adverse Drug Events Prevention Study Group,”JAMA, vol. 277, no. 4, pp. 307-311, Jan. 1997.

[14] L. L. Leape, A. G. Lawthers, T. A. Brennan, and W. G. Johnson, "Preventing medical injury," QRB Qual Rev Bull, vol. 19, no. 5, pp. 144-149, May 1993.

[15] “Infusion Pumps." [Online]. Available: http://www.fda.gov/MedicalDevices/ProductsandMedicalProcedures/GeneralHospitalDevices andSupplies/InfusionPumps/default.htm. [Accessed: 16-Dec-2013].

[16] “Block Diagram (SBD) - Infusion Pump - TI.com.” [Online]. Available: http://www.ti.com/solution/infusion_pump. [Accessed: 26-Nov-2013].

[17] P. Ingram and I. Lavery, "Peripheral intravenous therapy: key risks and implications for practice," Nursing Standard, vol. 19, no. 46, p. 55+, Jul. 2005.

[18] L. A. A. Gorski, "Infusion Nursing Standards of Practice.," Journal of infusion nursing, vol. 29, no. 1S, pp. 151-2, 2006.

[19] P. Carayon, A. S. Hundt, and T. B. Wetterneck, "Nurses' acceptance of Smart IV pump technology," International Journal of Medical Informatics, vol. 79, no. 6, pp. 401-411, Jun. 2010 .

[20] “ISMP Canada Home Page.” [Online]. Available: http://www.ismp-canada.org/index.htm. [Accessed: 05-Jul-2012].

[21] “MEDMARX Main Page.” [Online]. Available: https://www.medmarx.com/. [Accessed: 09Jul-2012].

[22] M. Colquhoun, I. S. Orrbine, L. Stromquist, C. Koczmara, R. Cheng, J. Greenall, B. Salsman, and S. Sabovitch, "National collaborative: top five drugs reported as causin harm through medication error in pediatrics," ISMP Canada, pp. 20-22, 2009.

[23] R. W. Hicks, S. C. Becker, D. Krenzischeck, and S. C. Beyea, "Medication errors in the PACU: a secondary analysis of MEDMARX findings," Journal of PeriAnesthesia Nursing, vol. 19, no. 1, pp. 18-28, Feb. 2004. 
[24] D. Sandlin, "Pediatric Medication Error Prevention," Journal of PeriAnesthesia Nursing, vol. 23, no. 4, pp. 279-281, Aug. 2008.

[25] J. Fanikos, K. Fiumara, S. Baroletti, C. Luppi, C. Saniuk, A. Mehta, J. Silverman, and S. Z. Goldhaber, "Impact of Smart Infusion Technology on Administration of Anticoagulants (Unfractionated Heparin, Argatroban, Lepirudin, and Bivalirudin)," The American Journal of Cardiology, vol. 99, no. 7, pp. 1002-1005, 2007.

[26] J. L. Wood and J. S. Burnette, "Enhancing patient safety with intelligent intravenous infusion devices: Experience in a specialty cardiac hospital," Heart \& Lung: The Journal of Acute and Critical Care, vol. 41, no. 2, pp. 173-176, Mar. 2012.

[27] T. Vanderveen and T. Riddle, "Infusion pump data documents high frequency of averted programming errors," Australian Critical Care, vol. 25, no. 2, pp. 141-142, May 2012.

[28] J. M. Rothschild, C. A. Keohane, E. F. Cook, E. J. Orav, E. Burdick, S. Thompson, J. Hayes, and D. W. Bates, "A controlled trial of smart infusion pumps to improve medication safety in critically ill patients," Crit. Care Med., vol. 33, no. 3, pp. 533-540, Mar. 2005.

[29] R. R. Maddox, S. Danello, C. K. Williams, and M. Fields, "Intravenous infusion safety initiative: Collaboration, evidencee-based best practices, and 'smart' technology help avert high-risk adverse drug event and improve patient outcomes," in Advances in Patient Safety: New Directions and Alternative Approaches, vol. 4, K. K. Henriksen, J. B. J. B. Battles, M. A. M. A. Keyes, and M. L. M. L. Grady, Eds. Rockville (MD): Agency for Healthcare Research and Quality (US), 2008.

[30] C. Gavriloff, "A Performance Improvement Plan to Increase Nurse Adherence to Use of Medication Safety Software," Journal of Pediatric Nursing, vol. 27, no. 4, pp. 375-382, Aug. 2012.

[31] M. Husch, C. Sullivan, D. Rooney, C. Barnard, M. Fotis, J. Clarke, and G. Noskin, "Insights from the sharp end of intravenous medication errors: implications for infusion pump technology," Qual Saf Health Care, vol. 14, no. 2, pp. 80-86, Apr. 2005.

[32] T. K. Nuckols, A. G. Bower, S. M. Paddock, L. H. Hilborne, P. Wallace, J. M. Rothschild, A. Griffin, R. J. Fairbanks, B. Carlson, R. J. Panzer, and R. H. Brook, "Programmable Infusion Pumps in ICUs: An Analysis of Corresponding Adverse Drug Events," J Gen Intern Med, vol. 23, no. Suppl 1, pp. 41-45, Jan. 2008.

[33] "Smart pumps need smart systems," Ontario critial incident learning, no. 7, pp. 1-2, Feb. 2014.

[34] “'Smart' Infusion Pumps are Selectively Intelligent.”[Online]. Available: http://www.fda.gov/MedicalDevices/Safety/AlertsandNotices/TipsandArticlesonDeviceSafety 
/ucm245160.htm. [Accessed: 11-Mar-2014].

[35] S. Manrique-Rodríguez, A. Sánchez-Galindo, C. M. Fernández-Llamazares, J. López-Herce, I. García-López, Á. Carrillo-Álvarez, and M. Sanjuro-Sáez, "Developing a drug library for smart pumps in a pediatric intensive care unit," Artificial Intelligence in Medicine, vol. 54, no. 3, pp. 155-161, Mar. 2012.

[36] D. W. Bates, "Variability in intravenous medication practices: implications for medication safety.," The joint commission journal on quality and patient safety, vol. 31, no. 4, pp. $203-$ 10, 2005.

[37] S. Manrique-Rodríguez, A. Sánchez-Galindo, C. M. Fernández-Llamazares, J. López-Herce, L. Echarri-Martínez, V. Escudero-Vilaplana, M. Sanjuro-Sáez, and Á. Carrillo-Álvarez, "Smart pump alerts: All that glitters is not gold," International Journal of Medical Informatics, vol. 81, no. 5, pp. 344-350, May 2012.

[38] A. D. Harding, "Use of Intravenous Smart Pumps for Patient Safety," Journal of Emergency Nursing, vol. 37, no. 1, pp. 71-72, Jan. 2011.

[39] M. Nunnally, C. P. Nemeth, V. Brunetti, and R. I. Cook, "Lost in Menuspace: User Interactions With Complex Medical Devices," IEEE Transactions on Systems, Man, and Cybernetics - Part A: Systems and Humans, vol. 34, no. 6, pp. 736-742, Nov. 2004.

[40] M. Luctkar-Flude, "Ending Infusion Confusion: Evaluating a Virtual Intravenous Pump Educational Module," Clinical Simulation in Nursing, vol. 8, no. 2, pp. e39-e48, Feb. 2012.

[41] S. Pauly-O’Neill, "Beyond the Five Rights: Improving Patient Safety in Pediatric Medication Administration Through Simulation," Clinical Simulation in Nursing, vol. 5, no. 5, pp. e181e186, Sep. 2009.

[42] A. K. Sowan, M. I. Gaffoor, K. Soeken, M. E. Johantgen, and V. U. Vaidya, "Impact of computerized orders for pediatric continuous drug infusions on detecting infusion pump programming errors: a simulated study," J Pediatr Nurs, vol. 25, no. 2, pp. 108-118, Apr. 2010.

[43] "Infusion Pumps > Infusion Pump Software Safety Research at FDA.” [Online]. Available: http://www.fda.gov/MedicalDevices/ProductsandMedicalProcedures/GeneralHospitalDevices andSupplies/InfusionPumps/ucm202511.htm. [Accessed: 11-Mar-2014].

[44] Association for the Advancement of Medication Instrumentation, "Infusing patients safely: Priority issues from the AAMI/FDA infusion device summit," Summit Proceedings, Oct. 2010.

[45] R. O. Duda, Pattern classification, 2nd ed. New York: Wiley, 2001. 
[46] D. Delen, A. Oztekin, and L. Tomak, "An analytic approach to better understanding and management of coronary surgeries," Decision Support Systems, vol. 52, no. 3, pp. 698-705, Feb. 2012.

[47] A. Wright, E. S. Chen, and F. L. Maloney, "An automated technique for identifying associations between medications, laboratory results and problems," Journal of Biomedical Informatics, vol. 43, no. 6, pp. 891-901, Dec. 2010.

[48] C.-L. Chang and C.-H. Chen, "Applying decision tree and neural network to increase quality of dermatologic diagnosis," Expert Systems with Applications, vol. 36, no. 2, Part 2, pp. 4035-4041, Mar. 2009.

[49] A. Vararuk, I. Petrounias, and V. Kodogiannis, "Data mining techniques for HIV/AIDS data management in Thailand," Journal of Enterprise Information Management, vol. 21, no. 1, pp. 52-70, 2008.

[50] C. C. Yang, W. T. Lin, H. M. Chen, and Y. H. Shi, "Improving scheduling of emergency physicians using data mining analysis," Expert Systems with Applications, vol. 36, no. 2, Part 2, pp. 3378-3387, Mar. 2009.

[51] J.-Y. Yeh, T.-H. Wu, and C.-W. Tsao, "Using data mining techniques to predict hospitalization of hemodialysis patients," Decision Support Systems, vol. 50, no. 2, pp. 439-448, Jan. 2011.

[52] J. de Oña, G. López, and J. Abellán, "Extracting decision rules from police accident reports through decision trees," Accident Analysis \& Prevention, vol. 50, pp. 1151-1160, Jan. 2013.

[53] D. Smadja, "Detection of Subclinical Keratoconus Using an Automated Decision Tree Classification,” vol. 156, no. 2, pp. 237-246.e1, Aug. 2013.

[54] “UCI Machine Learning Repository.” [Online]. Available: http://archive.ics.uci.edu/ml/. [Accessed: 02-Nov-2013].

[55] I. H. Witten, Data mining: practical machine learning tools and techniques, 3rd ed. Burlington, MA: Morgan Kaufmann, 2011.

[56] "Decision Trees and Decision Rules," in Data Mining, Hoboken, NJ, USA: John Wiley \& Sons, Inc., 2011, pp. 169-198.

[57] M., Bramer, Max, Bramer, Max Bramer, Principles of data mining. London: Springer, 2013.

[58] L. Breiman, "Technical note: Some properties of splitting criteria," Machine Learning, vol. 24, no. 1, pp. 41-47, Jul. 1996.

[59] “CHEO - About Us.” [Online]. Available: http://www.cheo.on.ca/en/About. [Accessed: 09Jul-2012]. 
[60] “Medfusion® 4000 Wireless Syringe Infusion Pump." [Online]. Available: http:/www.smiths-medical.com/catalog/syringe-pump/medfusion-4000-syringepump/medfusion-4000-wireless-syringe.html. [Accessed: 11-Jul-2012].

[61] “About Smiths Medical.” [Online]. Available: http://www.smiths-medical.com/aboutus/index.html. [Accessed: 28-Aug-2012].

[62] R. Sabrina, "6 Considerations Unique to Children's Hospitals in the Drive to Greater Healthcare Value." [Online]. Available: http://www.beckershospitalreview.com/strategicplanning/6-considerations-unique-to-childrens-hospitals-in-the-drive-to-greater-healthcarevalue.html. [Accessed: 27-Feb-2014].

[63] C. Dalton and M. Lupella, "Children's hospitals and the changing healthcare environment," Navigan Healthcare, 2013.

[64] P. healthcare alliance, "Pediatrics," 23-Apr-2009. [Online]. Available: https://www.premierinc.com/safety/topics/pediatrics/index.jsp. [Accessed: 27-Feb-2014].

[65] E. Fernandez, R. Perez, A. Hernandez, P. Tejada, M. Arteta, and J. Ramos, "Factors and Mechanisms for Pharmacokinetic Differences between Pediatric Population and Adults," Pharmaceutics, vol. 3, no. 4, pp. 53-72, Feb. 2011.

[66] C. K. Taketomo, J. H. Hodding, D. M. Kraus, I. Lexi-Comp, and American Pharmaceutical Association, Pediatric dosage handbook: including neonatal dosing, drug administration, \& extemporaneous preparations. Hudson, Ohio; [Washington, D.C.]: Lexi-Comp?; American Pharmaceutical Association, 2006.

[67] C. A. Repchinsky and Canadian Pharmacists Association, CPS 2012: compendium of pharmaceuticals and specialties?: the trusted Canadian drug reference for health professionals. Ottawa: Canadian Pharmacists Association, 2012.

[68] O. Ehrmann, "Les erreurs medicamenteuses en pediatrie hospitaliere," $\mathrm{PhD}$ of Pharmacy, Universite Claude Bernard, Lyon, 2012.

[69] K. N. Shaw, R. M. Ruddy, C. S. Olsen, K. A. Lillis, P. V. Mahajan, J. M. Dean, J. M. Chamberlain, and for the Pediatric Emergency Care Applied Research Network, "Pediatric Patient Safety in Emergency Departments: Unit Characteristics and Staff Perceptions," PEDIATRICS, vol. 124, no. 2, pp. 485-493, Jul. 2009.

[70] S. L. Bernstein, D. Aronsky, R. Duseja, S. Epstein, D. Handel, U. Hwang, M. McCarthy, K. John McConnell, J. M. Pines, N. Rathlev, R. Schafermeyer, F. Zwemer, M. Schull, B. R. Asplin, and Society for Academic Emergency Medicine, Emergency Department Crowding Task Force, "The Effect of Emergency Department Crowding on Clinically Oriented Outcomes," Academic Emergency Medicine, vol. 16, no. 1, pp. 1-10, Jan. 2009.

[71] C. P. Landrigan, J. M. Rothschild, J. W. Cronin, R. Kaushal, E. Burdick, J. T. Katz, C. M. 
Lilly, P. H. Stone, S. W. Lockley, D. W. Bates, and C. A. Czeisler, "Effect of Reducing Interns' Work Hours on Serious Medical Errors in Intensive Care Units," New England Journal of Medicine, vol. 351, no. 18, pp. 1838-1848, Oct. 2004.

[72] E. B. Kulstad, R. Sikka, R. T. Sweis, K. M. Kelley, and K. H. Rzechula, "ED overcrowding is associated with an increased frequency of medication errors," Am J Emerg Med, vol. 28, no. 3, pp. 304-309, Mar. 2010.

[73] J. I. Westbrook, A. Woods, M. I. Rob, W. T. M. Dunsmuir, and R. O. Day, “Association of interruptions with an increased risk and severity of medication administration errors," Arch. Intern. Med., vol. 170, no. 8, pp. 683-690, Apr. 2010.

[74] E. Kozer, D. Scolnik, A. Macpherson, T. Keays, K. Shi, T. Luk, and G. Koren, "Variables associated with medication errors in pediatric emergency medicine," Pediatrics, vol. 110, no. 4, pp. 737-742, Oct. 2002.

[75] R. Kaushal, D. W. Bates, C. Landrigan, K. J. McKenna, M. D. Clapp, F. Federico, and D. A. Goldmann, "Medication errors and adverse drug events in pediatric inpatients," JAMA, vol. 285, no. 16, pp. 2114-2120, Apr. 2001.

[76] C. Rowe, T. Koren, and G. Koren, "Errors by paediatric residents in calculating drug doses," Arch Dis Child, vol. 79, no. 1, pp. 56-58, Jul. 1998.

[77] J. Fordyce, F. S. j Blank, P. Pekow, H. A. Smithline, G. Ritter, S. Gehlbach, E. Benjamin, and P. L. Henneman, "Errors in a busy emergency department," Ann Emerg Med, vol. 42, no. 3, pp. 324-333, Sep. 2003.

[78] A. Kugelman, E. Inbar-Sanado, E. S. Shinwell, I. R. Makhoul, M. Leshem, S. Zangen, O. Wattenberg, T. Kaplan, A. Riskin, and D. Bader, "Iatrogenesis in Neonatal Intensive Care Units: Observational and Interventional, Prospective, Multicenter Study," PEDIATRICS, vol. 122, no. 3, pp. 550-555, Sep. 2008.

[79] P. J. Sharek, J. D. Horbar, W. Mason, H. Bisarya, C. W. Thurm, G. Suresh, J. E. Gray, W. H. Edwards, D. Goldmann, and D. Classen, "Adverse events in the neonatal intensive care unit: development, testing, and findings of an NICU-focused trigger tool to identify harm in North American NICUs," Pediatrics, vol. 118, no. 4, pp. 1332-1340, Oct. 2006.

[80] J. D. Rozich, "Adverse drug event trigger tool: a practical methodology for measuring medication related harm," Quality and Safety in Health Care, vol. 12, no. >3, pp. 194-200, Jun. 2003.

[81] T. A. Stavroudis, M. R. Miller, and C. U. Lehmann, "Medication errors in neonates," Clin Perinatol, vol. 35, no. 1, pp. 141-161, ix, Mar. 2008.

[82] S. Aranganayagi and K. Thangavel, "Improved K-Modes for Categorical Clustering using 
Weighted Dissimilarity Measure," International Journal of Information and Mathematical Sciences, p. 2009. 


\section{Appendix A}

\section{Event History Report Events}

Setup and Begin events for a PG infusion

\begin{tabular}{|c|c|c|}
\hline Setup & Therapy Event & $\begin{array}{l}\text { Profile: Hem/Onc } \\
\text { Category: Hem/Onc D-F } \\
\text { Drug Program Name: dimenhyDRINATE } 5 \\
\mathrm{mg} / \mathrm{mL}<50 \mathrm{~kg} \\
\text { Concentration: } 5 \\
\text { Concentration Units: } \mathrm{mg} / \mathrm{mL} \\
\text { Infusion Type: dose/ } \mathrm{kg} / \mathrm{time} \\
\text { Drug Usage Count: } 1\end{array}$ \\
\hline Begin & Therapy Event & $\begin{array}{l}\text { Drug Program Name: dimenhyDRINATE } 5 \\
\mathrm{mg} / \mathrm{mL}<50 \mathrm{~kg} \\
\text { Concentration: } 5 \\
\text { Concentration Units: } \mathrm{mg} / \mathrm{mL} \\
\text { Infusion Type: dose/ } \mathrm{kg} / \mathrm{time} \\
\text { Delivery Units: } \mathrm{mg} \\
\text { Syringe Model: B-D } \\
\text { Syringe Size: } 10 \mathrm{~mL} \\
\text { Weight: } 33 \\
\text { Dose: } 30 \\
\text { Main Rate: } 24 \\
\text { Time: } 00: 15: 00 \\
\text { Volume Limit: } 0 \\
\text { KVO Rate: } 0 \\
\text { PVD: } 0 \\
\text { Drug Usage Count: } 1\end{array}$ \\
\hline
\end{tabular}

Setup and Begin events for a non-PG infusion 


\begin{tabular}{|c|c|c|}
\hline Setup & Therapy Event & $\begin{array}{l}\text { Profile: Hem/Onc } \\
\text { Infusion Type: Volume over Time } \\
\text { Drug Usage Count: } 2\end{array}$ \\
\hline Begin & Therapy Event & $\begin{array}{l}\text { Infusion Type: Volume over Time } \\
\text { Syringe Model: B-D } \\
\text { Syringe Size: } 10 \mathrm{~mL} \\
\text { Main Rate: } 30 \\
\text { Volume: } 5 \\
\text { Time: } 00: 10: 00 \\
\text { Volume Limit: } 0 \\
\text { KVO Rate: } 0 \\
\text { PVD: } 0 \\
\text { Drug Usage Count: } 2\end{array}$ \\
\hline
\end{tabular}

Soft limit override event

\begin{tabular}{|l|l|}
\hline & Profile: Hem/Onc \\
& Category: Hem/Onc D-F \\
& Drug Program Name: dexamethasone 1 \\
$\mathrm{mg} / \mathrm{mL}$ & \\
& Message: Pharmguard Change \\
& Parameter: Main Time \\
& Original Value: 00:00:00 \\
& Entered Value: $20: 00: 00$ \\
& Final Value: $20: 00: 00$ \\
& Upper Soft Limit: 00:30:00 \\
Override Pharmguard Change & Lower Soft Limit: 00:10:00
\end{tabular}

Soft limit warning event

\begin{tabular}{|l|l|}
\hline & Profile: Emergency Dept \\
& Category: Emergency Dept M-O \\
& Drug Program Name: metoclopramide 0.5 \\
mg/mL Inter & \\
& Message: Pharmguard Change \\
& Parameter: Main Time \\
& Original Value: 00:00:00 \\
& Entered Value: 20:00:00 \\
& Final Value: 00:20:00 \\
& Upper Soft Limit: 00:30:00 \\
Warning & Lower Soft Limit: 00:15:00 \\
\hline
\end{tabular}

Hard limit event 


\begin{tabular}{|l|l|}
\hline & Profile: Hem/Onc \\
& Category: Hem/Onc A-C \\
& Drug Program Name: amphotericin B \\
& liposomal $2 \mathrm{mg} / \mathrm{m}$ \\
& Message: Pharmguard Change \\
& Parameter: Dose \\
& Parameter Units: $\mathrm{mg}$ \\
& Original Value: 8.0000 \\
& Entered Value: 45.0000 \\
\hline Hardlimit & Final Value: 8.0000 \\
\hline
\end{tabular}

